\title{
Review Article \\ Synthesis and Reactions of Five-Membered Heterocycles Using Phase Transfer Catalyst (PTC) Techniques
}

\author{
Ahmed M. El-Sayed, ${ }^{1}$ Omyma A. Abd Allah, ${ }^{1}$ \\ Ahmed M. M. El-Saghier, ${ }^{1}$ and Shaaban K. Mohamed $^{2,3}$ \\ ${ }^{1}$ Department of Chemistry, Faculty of Science, Sohag University, Sohag 82524, Egypt \\ ${ }^{2}$ Chemistry and Environmental Division, Manchester Metropolitan University, Manchester M1 5GD, UK \\ ${ }^{3}$ Chemistry Department, Faculty of Science, Minia University, El-Minia 61519, Egypt \\ Correspondence should be addressed to Omyma A. Abd Allah; omymatif66@yahoo.com
}

Received 1 May 2013; Revised 15 September 2013; Accepted 28 October 2013; Published 25 February 2014

Academic Editor: Jorge F. Fernandez-Sanchez

Copyright (C) 2014 Ahmed M. El-Sayed et al. This is an open access article distributed under the Creative Commons Attribution License, which permits unrestricted use, distribution, and reproduction in any medium, provided the original work is properly cited.

Phase transfer catalysts (PTCs) have been widely used for the synthesis of organic compounds particularly in both liquid-liquid and solid-liquid heterogeneous reaction mixtures. They are known to accelerate reaction rates by facilitating formation of interphase transfer of species and making reactions between reagents in two immiscible phases possible. Application of PTC instead of traditional technologies for industrial processes of organic synthesis provides substantial benefits for the environment. On the basis of numerous reports it is evident that phase-transfer catalysis is the most efficient way for generation and reactions of many active intermediates. In this review we report various uses of PTC in syntheses and reactions of five-membered heterocycles compounds and their multifused rings.

\section{Introduction}

Organic synthesis is an essential way to get chemical products having practical applications such as pharmaceuticals, plant protection agents, dyes, photographic sensitizers, and monomers. Transformations of starting materials into desired final products usually require number of chemical operations in which additional reagents, catalysts, and solvents are employed. Thus, during any synthetic method, besides the desired products, many waste materials are produced because transformations of reactants into products are neither quantitative nor selective processes. This waste could be regenerated, destroyed, or disposed. This will lead to consuming much energy and creating heavy burden on the environment. Therefore it is of a great importance to develop and use synthetic methodologies that minimize or eliminate such problems. One of the most common and efficient methodologies that fulfill this requirement is employing phase-transfer catalyses techniques [1-4]. The most significant advantages of use of PTCs in industrial applications are [5]
(1) elimination of organic solvents,

(2) elimination of dangerous, inconvenient, and expensive reagents such as $\mathrm{NaH}$ and $\mathrm{NaNH}_{2}$,

(3) increasing the reactivity and selectivity of the active species,

(4) improving the yield and purity of products to the optimum records,

(5) simplifying the whole synthetic process and making it safer and objective,

(6) reducing industrial wastes and overall costs and saving energy which gives a positive impact on economic and environmental interests,

(7) accelerating and performing mimic reactions in an efficient mode.

1.1. Fundamentals of Phase Transfer Catalysis (PTC). First phase transfer catalysis was discovered by Jarrouse and Hebd in 1951 when they observed that the quaternary ammonium 


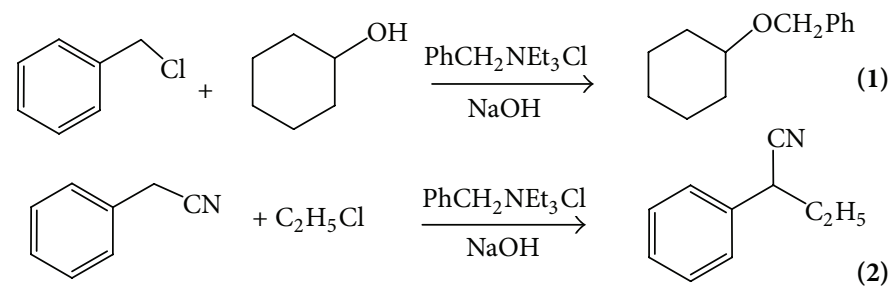

Figure 1

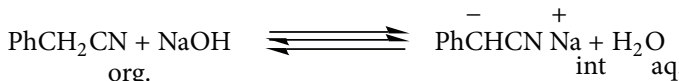

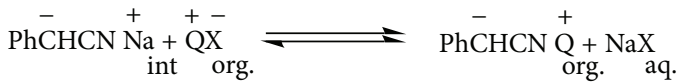

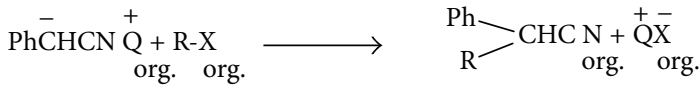

Figure 2

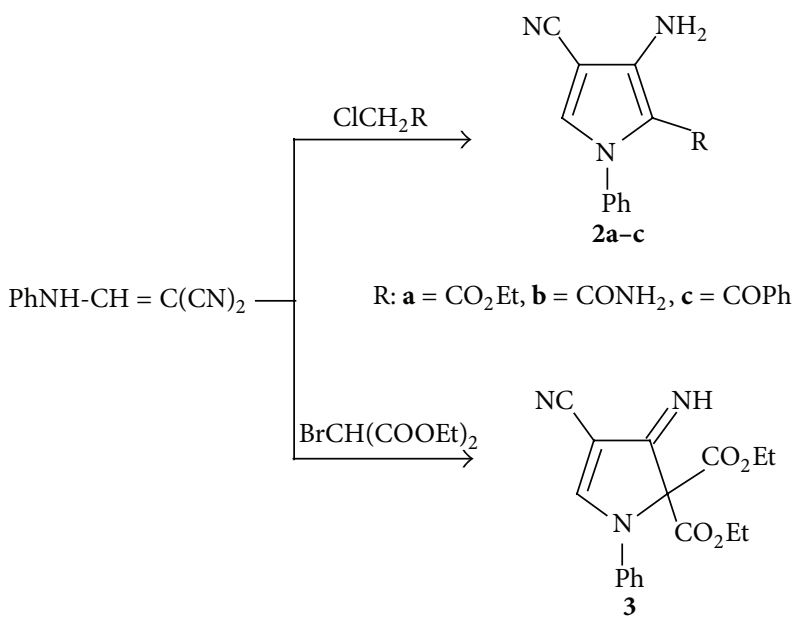

Figure 3

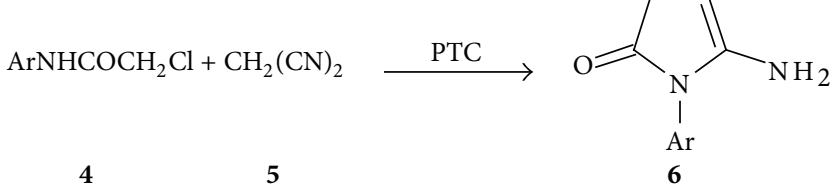

FIGURE 4

salt and benzyltriethylammonium chloride accelerated twophase reaction of benzyl chloride with cyclohexanol (Figure 1; (1)) and the two-phase alkylation reaction of phenylacetonitrile with benzyl chloride or ethyl chloride [6] (Figure 1; (2)).

In addition, numerous publications and patents [7-12] have been reported during the period of 1950-1965. PTCs techniques have been further developed by Makosza et al. for the purpose of obtaining more efficient and pure yield [13$16]$.
1.2. Mechanism of Phase-Transfer Catalysis. All phasetransfer catalyzed reactions involve at least two steps:

(1) transfer of one reagent from its ground phase into the second phase as an intermediate,

(2) reaction of the transferred reagent with the nontransferred reagent, for example, the alkylation of phenylacetonitrile with alkyl halide using aqueous $\mathrm{NaOH}$ as a base and tetrabutylammonium halide 


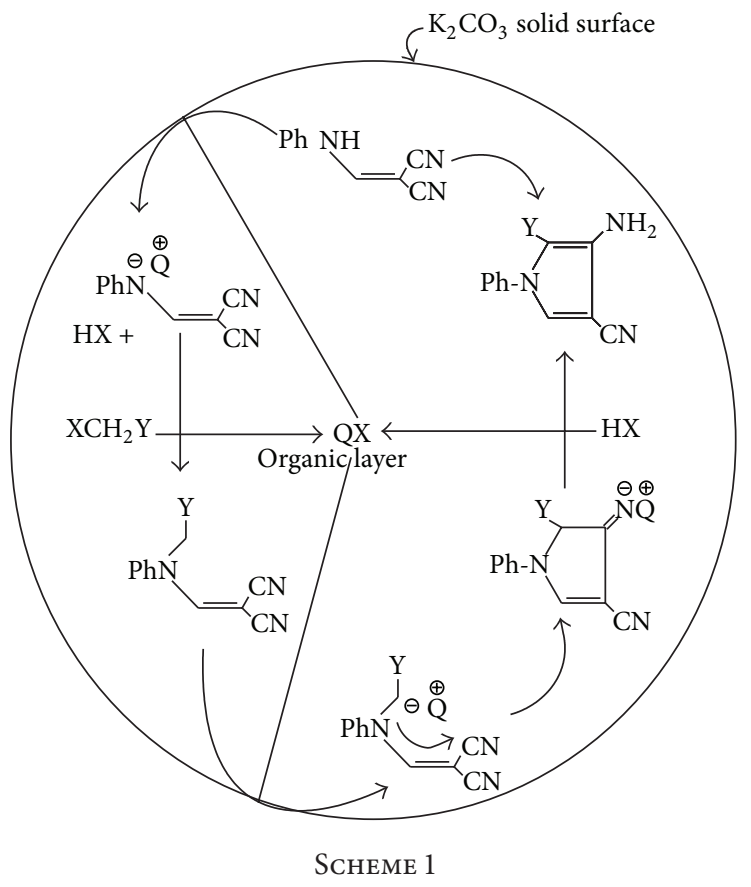

(QX) as a catalyst can be formulated as shown in Figure 2.

The concept of phase-transfer catalysis is not limited to anion transfer but is much more general, so that, in principle, one could also transfer cations, free radicals, or whole molecule. Phase transfer catalysis is classified as liquid-liquid, liquid-solid, liquid-gas, solid-gas, or solid-solid systems.

\section{Application of Phase Transfer Catalysis in Synthesis of Five-Membered Ring of Heterocyclic Compounds}

2.1. Synthesis of Five-Membered Ring Heterocycles Containing One Heteroatom. Treatment of anilinomethylenemalononitrile 1 with ethyl chloroacetate, chloroaceta-mide, phenacyl chloride, or diethyl bromomalonate in $1: 1: 1$ molar ratio (dioxan $/ \mathrm{K}_{2} \mathrm{CO}_{3}$ /tetrabutylammonium bromide (TBAB)) afforded the corresponding substituted pyrroles $2 \mathbf{a}-$ c and 3, respectively [17] (Figure 3).

The catalytic cycle which explains the reaction pathway can be simplified as in Scheme 1.

A simple and convenient synthesis of 2-amino-1-aryl-5oxo-4,5-dihydro- $1 H$-pyrrole-3-carbonitriles 6 was achieved by reaction of $\mathrm{N}$-aryl-2-chloroacetamide 4 and malononitrile 5 under solid-liquid PTC condition $\left(\mathrm{CH}_{3} \mathrm{CN} / \mathrm{K}_{2} \mathrm{CO}_{3} / 18\right.$ crown-6) at room temperature [18] (Figure 4).

3-Amino-4-ethoxycarbonyl-1-phenyl-5-thioxo-2,5-dihydro-2-pyrrolidene malononitrile 9 a was prepared via reaction of malononitrile 5, phenyl isothiocyanate 7 , and ethoxymethylenemalononitrile $\mathbf{8 a}$ in 1:1:1 molar ratio under the PTC mixture of dioxan $/ \mathrm{K}_{2} \mathrm{CO}_{3} /(\mathrm{TBAB})$. Replacing of ethyl ethoxymethylenecyanoacetate $\mathbf{8 b}$ with $\mathbf{8 a}$ gave ethyl[3-amino-4-ethoxy-carbonyl-1-phenyl-5-thioxo-2,5-dihydro-2-pyrrolidene]cyanoacetate $\mathbf{9 b}$ and $\mathbf{1 0}$ under the same experimental conditions [19] (Figure 5).

Moreover, the reaction of ethyl cyanoacetate $\mathbf{1 1}$ with phenyl isothiocyante 7 and ethoxymethylenemalononitrile $\mathbf{8 a}$ or ethyl ethoxymethylenecyanoacetate $\mathbf{8 b}$ in $1: 1: 1$ molar ratio under same PTC experimental conditions afforded the corresponding pyrrole derivatives $12 \mathbf{a}, \mathbf{b}$, and 13 [19] (Figure 6).

Reaction of methyl 2,5-dibromopentanoate 14 with trichloroacetamide 15 under solid-liquid technique $\left(\mathrm{CH}_{3} \mathrm{CN} /\right.$ $\mathrm{K}_{2} \mathrm{CO}_{3}$ /TEBACl) yielded methyl $\mathrm{N}$-trichloroacetyl-2-pyrrolidine carboxylate 16 [20] (Figure 7).

Substituted pyrrolidines $\mathbf{1 9}$ were synthesized via the 1,3-dipolar cycloaddition reaction between imino esters $\mathbf{1 7}$ (derived from alanine and glycine with alkanes) and alkyl acrylate $\mathbf{1 8}$ in a mixture of THF or toluene, $\mathrm{KOH}$, and TBACl [21] (Figure 8).

1,4-Di(malononitrilemethyleneamino)benzene $\mathbf{2 0}$ was allowed to react with ethyl chloroacetate, chloroacetamide, phenacyl chloride, or diethyl bromomalonate in $1: 2$ molar ratios under solid-liquid PTC technique to give the corresponding 1,4-bi(1-pyrrolyl) benzene derivatives 21a-c and 22 [17] (Figure 9).

Pyrrolidine derivatives 24 were obtained with thiophenes 25 from the reaction of diethyl cyanomalonate 23 with phenyl isothiocyanate 7 and chloroacetonitrile or chloroacetamide using the PTC of (DMF/ $\left.\mathrm{K}_{2} \mathrm{CO}_{3} / \mathrm{TBAB}\right)$ [22] (Figure 10). 

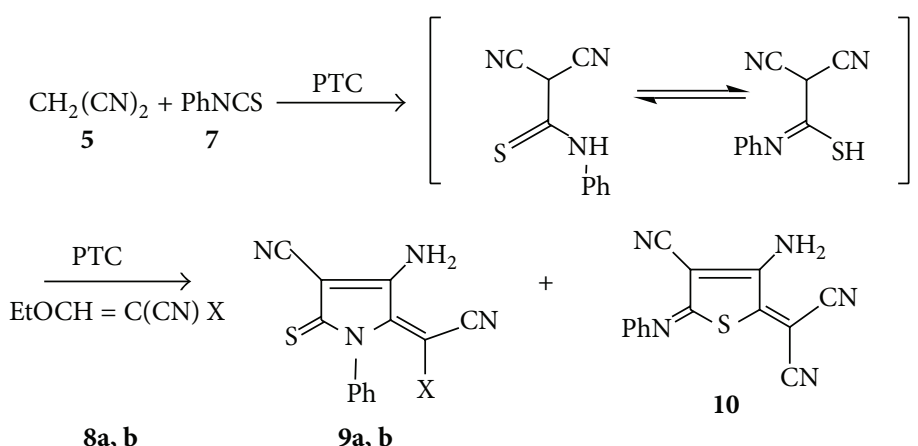

8a, b

9a, b

$\mathrm{X}: \mathbf{a}=\mathrm{CN}, \mathbf{b}=\mathrm{COOEt}$

Figure 5

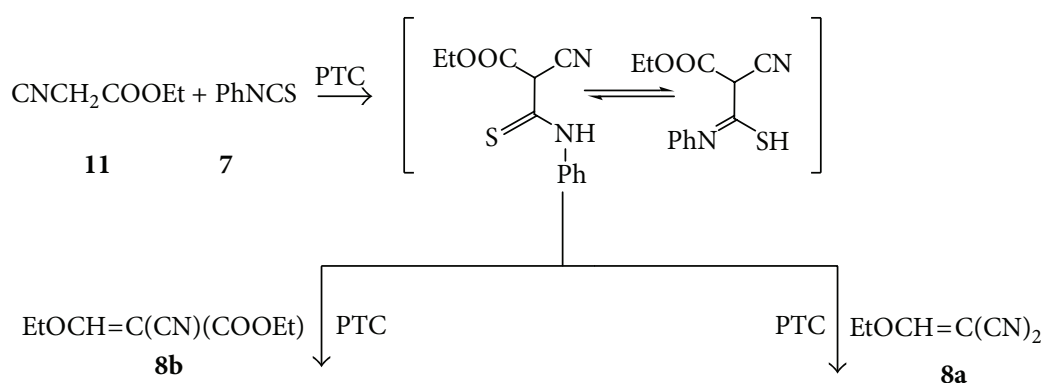

$8 b$<smiles>CCOC(=O)C(C#N)=C1SC(=N)C(C(=O)OCC)=C1N</smiles>

$\mathrm{H}_{2} \mathrm{O} / \mathrm{K}_{2} \mathrm{CO}_{3} \mid \begin{aligned} & \text { Hydrolysi } \\ & -\mathrm{PhNH}_{2}\end{aligned}$<smiles>CCOC(=O)C(C#N)=C1SC(=O)C(C(=O)OCC)=C1N</smiles>

13

FIGURE 6<smiles>CCOC(=O)/C(C#N)=C1\C(N)=C(C=O)C(=S)N1c1ccccc1</smiles>

12b<smiles>CCOC(=O)C1=C(N)C(=C(C#N)C#N)N(c2ccccc2)C1=S</smiles>

12a
14

15

$\mathrm{Br}\left(\mathrm{CH}_{2}\right)_{3} \mathrm{CH}(\mathrm{Br}) \mathrm{COOCH}_{3}+\mathrm{Cl}_{3} \mathrm{CCONH}_{2} \stackrel{\mathrm{PTC}}{\longrightarrow}$<smiles>COC(=O)C1CCCN1C(=O)C(Cl)(Cl)Cl</smiles>

16

Figure 7

One-pot reaction of malononitrile $\mathbf{5}$, carbon disulphide, and chloroacetamide in 1:1:1 molar ratio under solidliquid PT catalysis (benzene/ $\mathrm{K}_{2} \mathrm{CO}_{3} / \mathrm{TBAB}$ ) has furnished 3-amino-2-carboxyamido-4-cyano-5-mercaptothiophene 26 [23] (Figure 11). Also, treatment of malononitrile 5 with
$\mathrm{CS}_{2}$ and ethyl chloroacetate under the same experimental conditions gave the corresponding thiophene derivative $\mathbf{2 7}$ [23] (Figure 11).

Different thiophene compounds (24 and 28-30) prepared from reaction of diethyl cyanomalonate 23 with carbon 


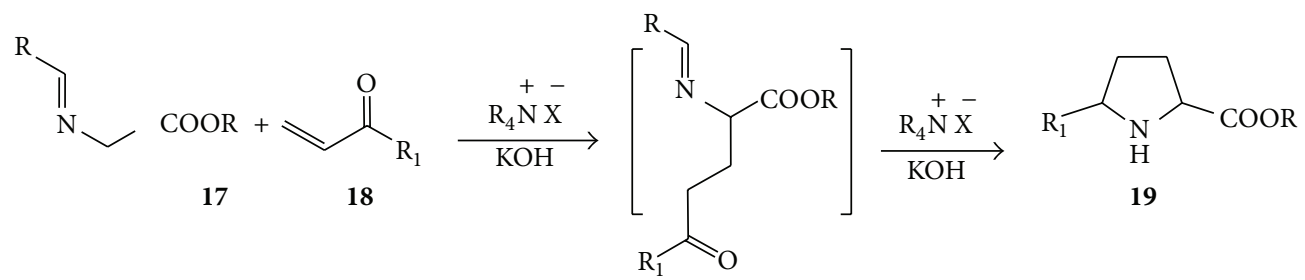

Figure 8

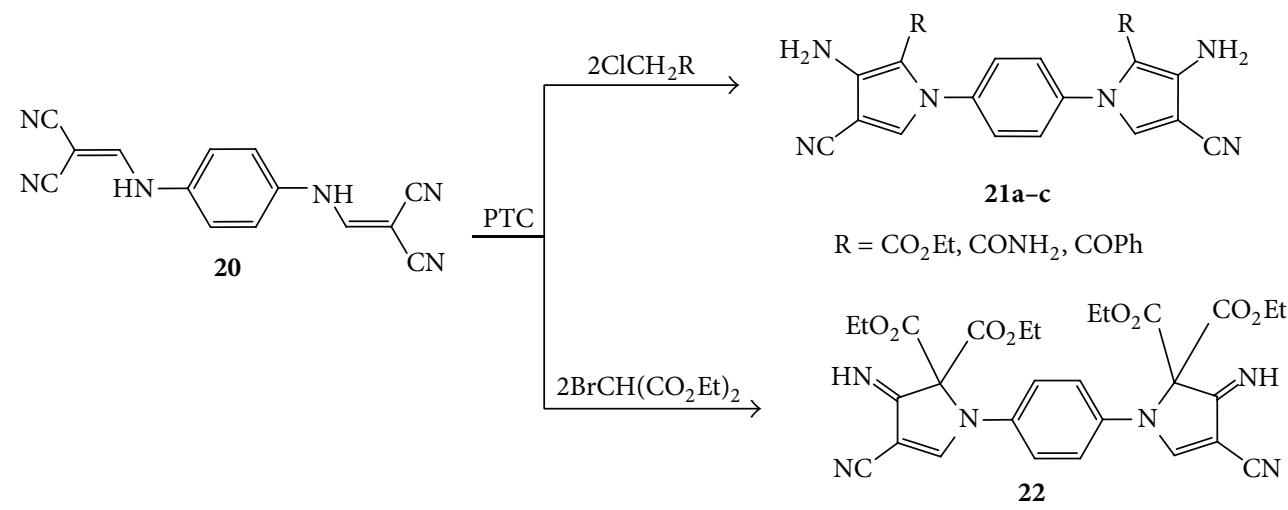

Figure 9
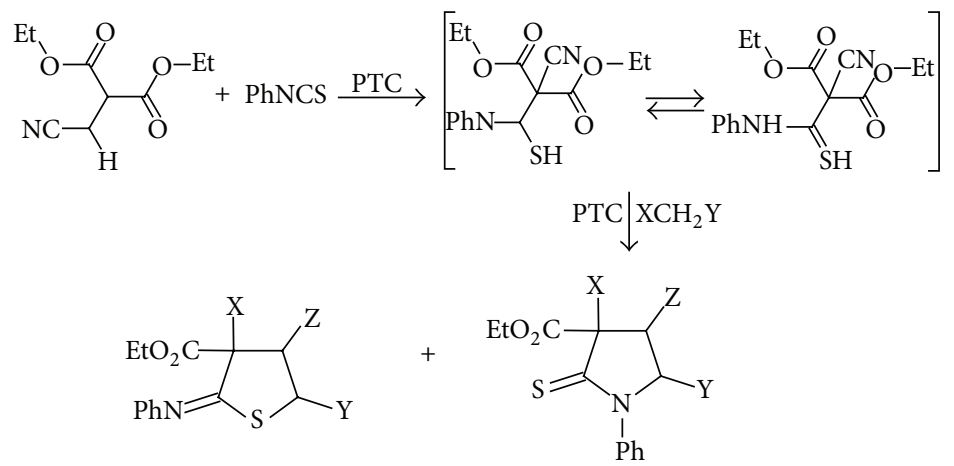

25

24a-c

$\mathrm{X}=\mathrm{CO}_{2} \mathrm{Et}, \mathrm{Y}=\mathrm{CN}, \mathrm{Z}=\mathrm{NH}_{2} \quad$ 24a, $\mathbf{b}: \mathrm{X}=\mathrm{CO}_{2} \mathrm{Et}, \mathrm{Y}=\mathrm{CN}, \mathrm{Z}=\mathrm{NH}_{2}$

24c: $\mathrm{X}=\mathrm{CN}, \mathrm{Y}=\mathrm{CONH}_{2}, \mathrm{Z}=\mathrm{OH}$

Figure 10
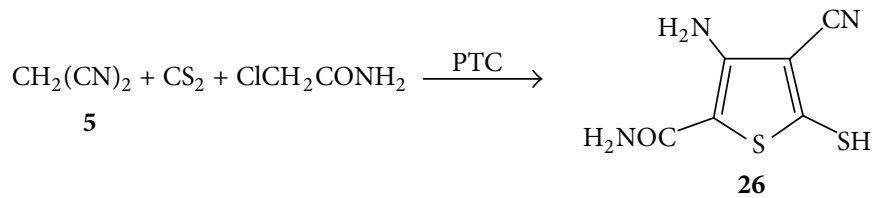

$\mathrm{CH}_{2}(\mathrm{CN})_{2}+\mathrm{CS}_{2}+\mathrm{ClCH}_{2} \mathrm{COOEt} \stackrel{\text { PTC }}{\longrightarrow}$

5

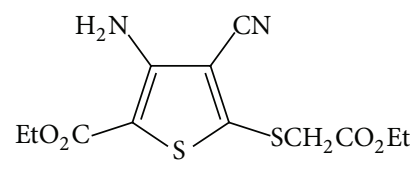

27

Figure 11 

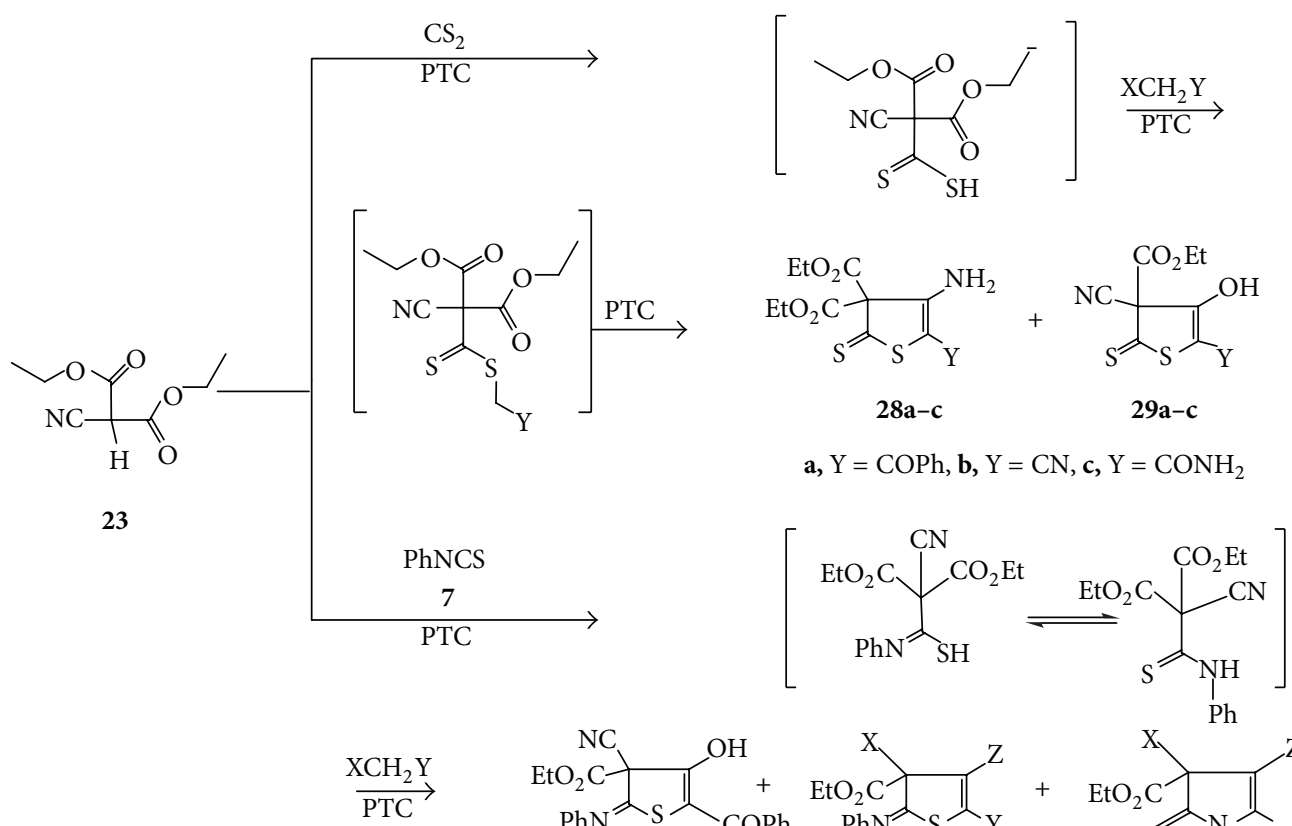<smiles>CCOC(=O)C1=C(O)C(C(=O)Oc2ccccc2)C(C#N)=C1[PH2+]</smiles>

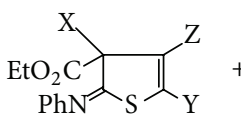<smiles>[Y]C1=C([Z])C2([Y])C(=S)N([Pb])C(=O)C12</smiles>

30 $25 \mathrm{a}-\mathrm{c}$ 24

24a, b, 25a-b; $\mathrm{X}=\mathrm{CO}_{2} \mathrm{C}_{2} \mathrm{H}_{5}, \mathrm{Y}=\mathrm{CN}, \mathrm{Z}=\mathrm{NH}_{2}$ 24c, 25c; $\mathrm{X}=\mathrm{CO}_{2} \mathrm{C}_{2} \mathrm{H}_{5}, \mathrm{Y}=\mathrm{CONH}_{2}, \mathrm{Z}=\mathrm{NH}_{2}$

FIGURE 12

disulphide or phenyl isothiocyanate 7 along with different active halocompounds under solid-liquid phase condition (dioxan $/ \mathrm{K}_{2} \mathrm{CO}_{3} / \mathrm{TBAB}$ ) have been reported by Abd Allah and El-Sayed [22] (Figure 12).

2-Thiophenylidenes 31-33 were obtained through the reaction of either malononitrile 5 or ethyl cyanoacetate $\mathbf{1 1}$ with carbon disulphide along with ethoxymethylenemalononitrile $\mathbf{8 a}$ or ethyl ethoxymethylenecyanoacetate $\mathbf{8 b}$ using liquid-solid technique (dioxan $/ \mathrm{K}_{2} \mathrm{CO}_{3} / \mathrm{TBAB}$ ) [19] (Figure 13).

In this reaction, the yield of products was found to be a temperature dependent. Thus, at low temperature $\left(40^{\circ} \mathrm{C}\right)$ it affords the aminothiophene derivative 32a in high yield while at high temperature hydroxythiophene $\mathbf{3 2} \mathbf{b}$ or $\mathbf{3 3 b}$ was the solely product but in low yield.

The reaction of pyridosultam 34 with 3-chloropropyl thiocyanate under the experimental condition (toluene/aq. $\mathrm{NaOH} / \mathrm{TBAB}$ ) yielded the corresponding dihydrothiophene derivative 35 [24] (Figure 14).

4-Amino-5-cyano-2-hydroxy-3-furancarboxylic acid 37 and compound 38 were synthesized by treating 1-phenyl-3,5pyrazolinedione 36 with bromomalononitrile under solidliquid condition (dioxan/ $\mathrm{K}_{2} \mathrm{CO}_{3} / \mathrm{TBAB}$ ) (Figure 15) [25].

The reaction pathway for the formation of unexpected compound 37 was assumed to proceed via catalytic hydrolysis of compound 38 into the furan derivative 37 and phenyl hydrazine [25] (Figure 16).
Condensation of salicylaldehyde 39 and its derivatives with various esters of chloroacetic acids 40 in the presence of TBAB led to the synthesis of benzo[b]furans 41 under solventless and microwave irradiation technique [26] (Figure 17).

Liquid-liquid PTC reaction of 4-chlorobutyronitrile $\mathbf{4 2}$ with nonenolizable aldehydes 43 via sequence of an addition cyclization reaction gave tetrahydrofuran-3-carbonitrile derivative 44 [27] (Figure 18).

Fused bis-aroylbenzodifurans 46 have been synthesized by condensing 2,4-diacetyl resorcinol 45 with various psubstituted phenacyl bromides [28] (Figure 19).

Methyl or ethyl 3-amino-4-arylthiophenes-2-carboxylates $48 \mathbf{a}-\mathbf{f}$ were synthesized by Thorpe reaction through the treatment of 3-hydroxy-2-arylacrylonitriles $47 \mathbf{a}-\mathbf{f}$ and methyl or ethyl thioglycolates with hydrochloric acid by using different PTC conditions (solid-liquid or liquid-liquid). The solid-liquid PTC conditions using 18-crown-6 along with potassium hydroxide as a catalyst are the method with excellent yields. The reactions were carried out in acetonitrile at RT [29] (Figure 20).

In Japp-Klingemann reaction the indole derivatives $\mathbf{5 2}$ have been prepared as indicated in Figure 21. The addition of a suitable PTC catalyst to the reaction could significantly improve the reaction process. Firstly, aryl amines 49 were diazotized and reacted under alkaline conditions ethyl 2-[2-(1,3-dioxo-1,3-dihydro- $2 \mathrm{H}$-isoindol-2-yl)ethyl]-3oxobutanoate 50 using PTC-promoted Japp-Klingemann 


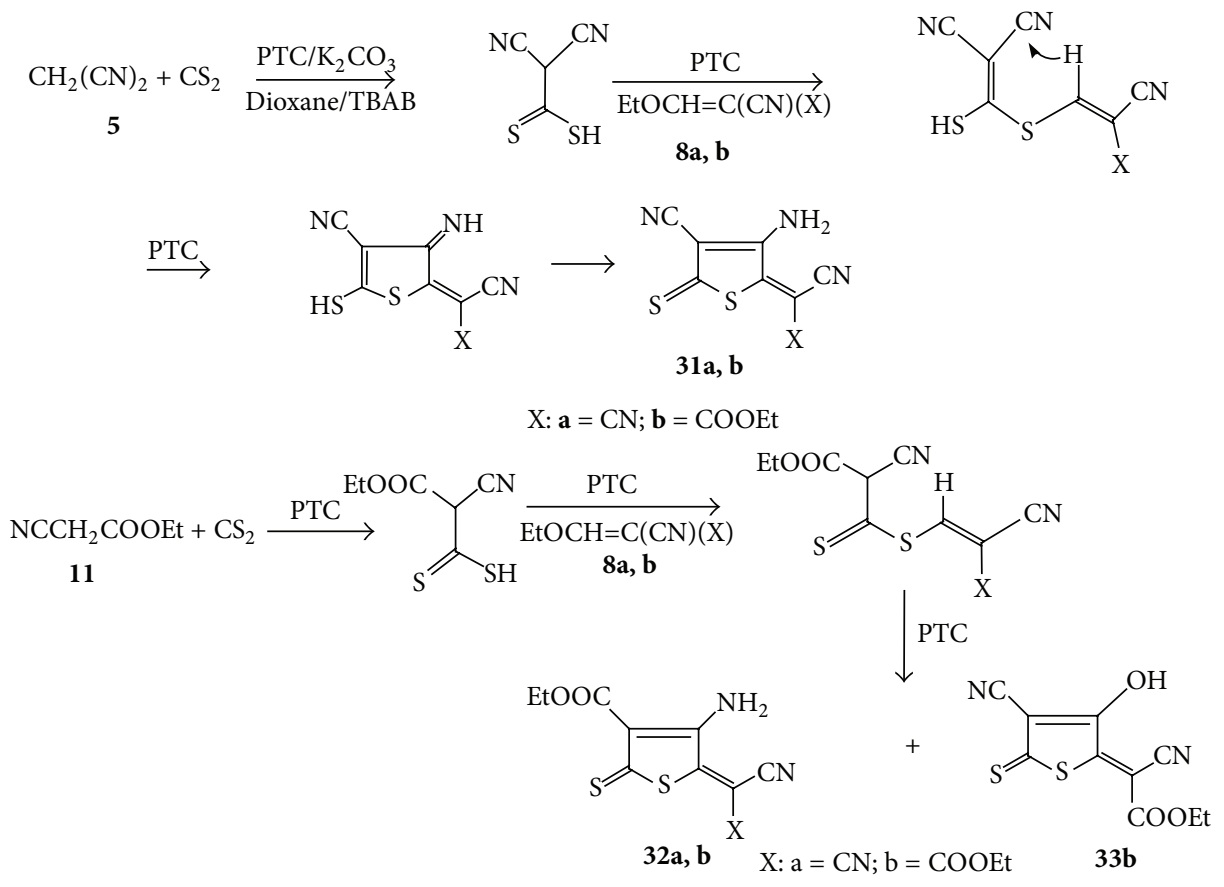

FIGURE 13

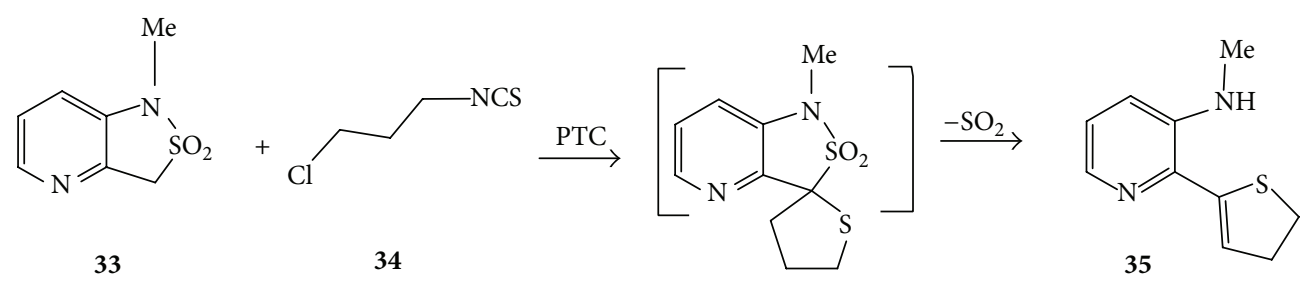

FIGURE 14

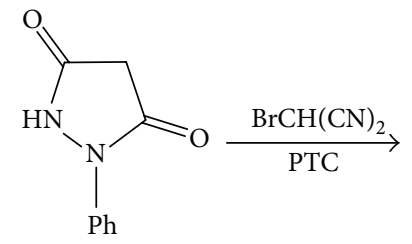

36<smiles>N#Cc1c(N)oc(O)c1C(=O)O</smiles>

37<smiles>N#Cc1c(N)oc2c1c(=O)[nH]n2-c1ccccc1</smiles>

38

Figure 15<smiles>N#Cc1c(N)oc2c1c(=O)[nH]n2-c1ccccc1</smiles>

38<smiles>N#Cc1c(N)oc(N(N)c2ccccc2)c1C(=O)O</smiles>

Figure 16<smiles>N#Cc1c(N)oc(O)c1C(=O)O</smiles>

37 
<smiles>OC1=CC=[R]#CC=C1C=[V]</smiles>

39

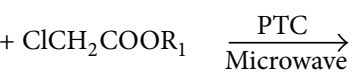

40<smiles>[R][X]c1ccc2oc(C(=O)OCC)cc2c1</smiles>

41

FIGURE 17<smiles>COCC=[N+]CCCCl</smiles><smiles>CCCC(O)C(C#N)CCCl</smiles>

44

FIgURE 18<smiles>CC(=O)c1ccc(O)c(C(C)=O)c1O</smiles>

45

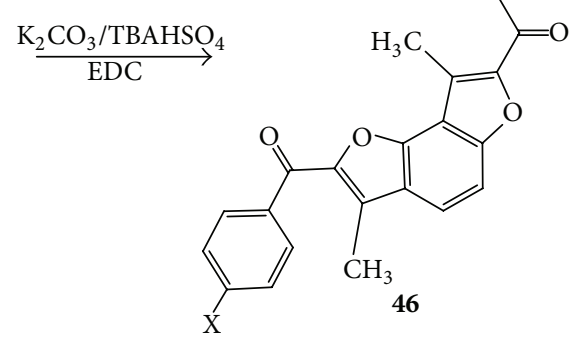

$$
\mathrm{X}=\mathrm{H}, \mathrm{Cl}, \mathrm{NO}_{2}, \mathrm{CH}_{3}, \mathrm{OCH}_{3}, \mathrm{OCH}_{3}, \mathrm{Br}
$$

FIgURe 19

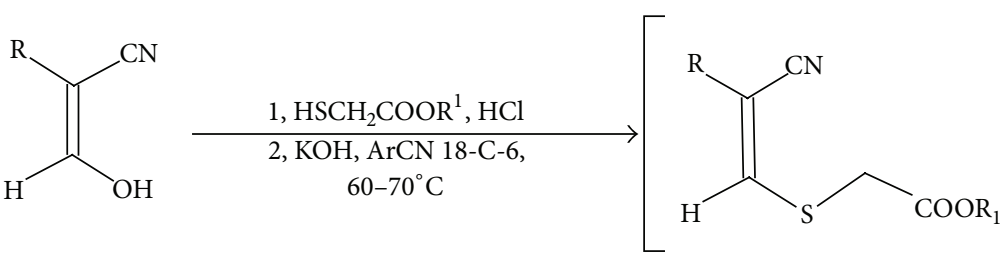

47a-f

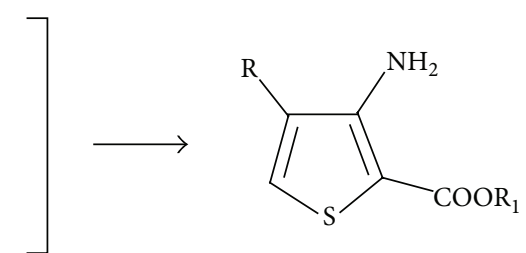

48a-f

$\begin{array}{ll}\mathrm{R} & \mathrm{R}^{1} \\ \text { a, ph } & \text { COOMe } \\ \text { b, 4-O- } \mathrm{CH}_{3} & \text { COOMe } \\ \text { c, benzo[b]furyl-2 } & \text { COOMe } \\ \text { d, thinyl-2 } & \text { COOMe } \\ \text { e, dimethyl 2,5-thinyl } & \text { COOMe } \\ \text { f, } \mathrm{C}_{6} \mathrm{H}_{5} & \text { COOEt }\end{array}$

FIGURE 20 reaction to form the ring-opened aryl hydrazones 51. These aryl hydrazones were cyclized and converted into the corresponding indole derivatives 52 by adding hydrochloric acid in ethanol [30] (Figure 21).

Synthesis of dimethyl phosphinyl substituted tetrahydropyrroles 55 via 1,3-cycloaddition reaction of the azomethine 53 to electron deficient alkenes such as $\alpha, \beta$-unsaturated ketones (e.g., benzylideneacetophenone 54), esters, and nitriles took place in high yield and low diastereoselectivity in phase-transfer catalysis conditions (solid potassium carbonate, triethylbenzylammonium chloride (TEBA)) in acetonitrile as a solvent [31] (Figure 22).

When Schiff base 53 and ethyl cinnamate 56 reacted under different phase-transfer catalysis conditions (10 equivalents of aqueous $\mathrm{NaOH}(50 \%) / \mathrm{TEBA} / \mathrm{DMSO})$, the ester of pyrrolidinecarboxylic acid $\mathbf{5 7}$ has not been formed; 


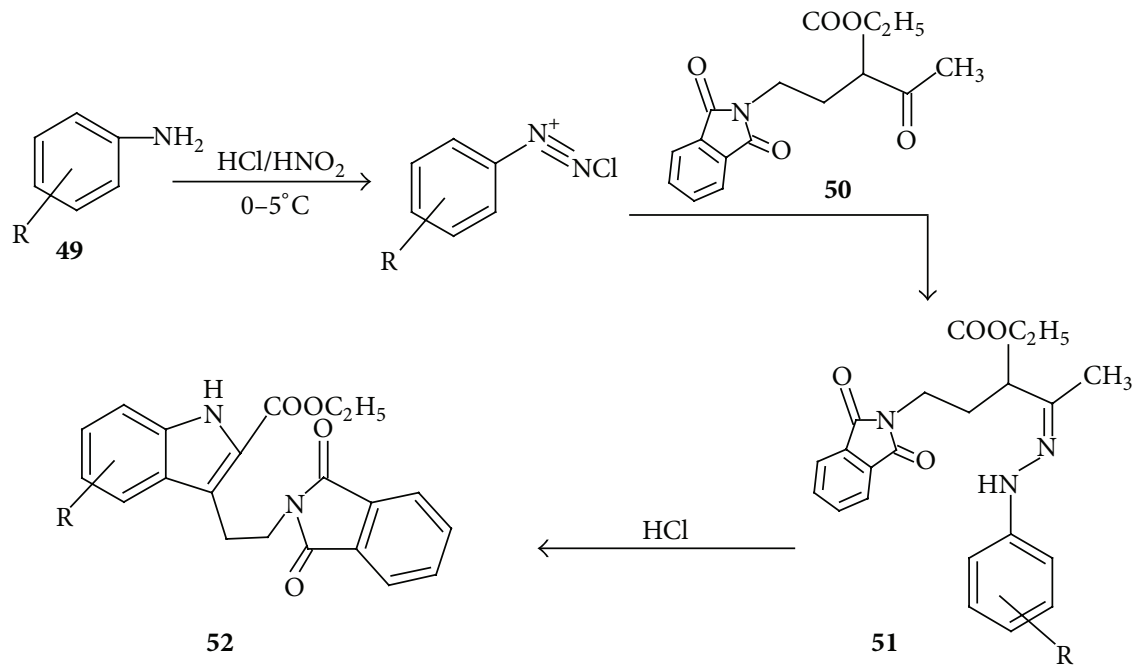

FIgURE 21<smiles>CP(C)(=O)CN=Cc1ccc(Cl)cc1</smiles>

$+$<smiles>O=C(/C=C/c1ccccc1)c1ccccc1</smiles>

54<smiles>C[C@@H]1C(C(=O)c2ccccc2)C(c2ccc(Cl)cc2)NC1P(C)(C)=O</smiles>

55

Figure 22

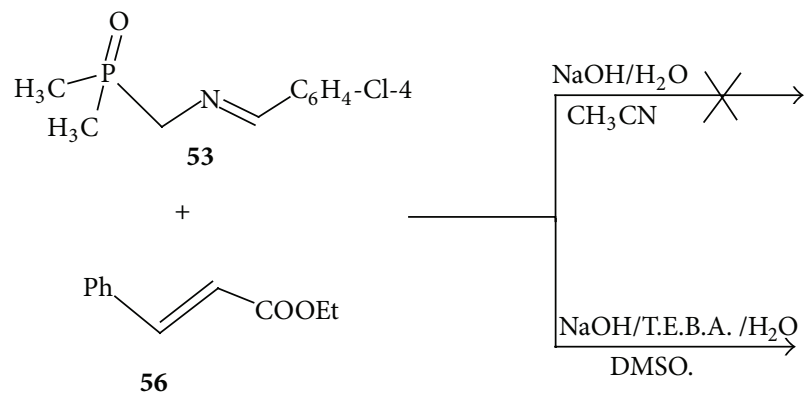<smiles>CCCC1NC(P(C)(C)=O)C(C)C1C(=O)OCC</smiles><smiles>C[C@H]1NC([P+](C)(C)=O)C([CH]Cl)C1C(=O)O</smiles>

FIGURE 23

instead the acid itself $\mathbf{5 8}$ was obtained as a mixture of two diastereoisomers [31] (Figure 23).

2.2. Synthesis of Five-Membered Ring Heterocycles Containing Two Heteroatoms. (4Z)-4-(4-amino-1,3-dithiol-2-ylidene)-5-methyl-2-phenyl-2,4-dihydro-3H-pyrazol-3-one derivative 60 was the product of a reaction of 3-methyl1-phenyl-2-pyrazoline-5-one 59 with $\mathrm{CS}_{2}$ and chloroacetonitrile in equimolar ratio under solid-liquid technique (benzene/ $\mathrm{K}_{2} \mathrm{CO}_{3} / \mathrm{TBAB}$ ) [23] (Figure 24).

Similarly, 1-phenyl-3,5-pyrazolidinedione 36 was treated with $\mathrm{CS}_{2}$ and ethyl chloroacetate under the same condition 


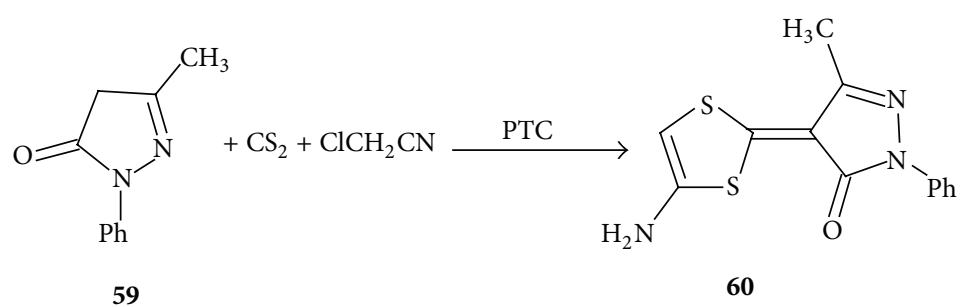

FIGURE 24

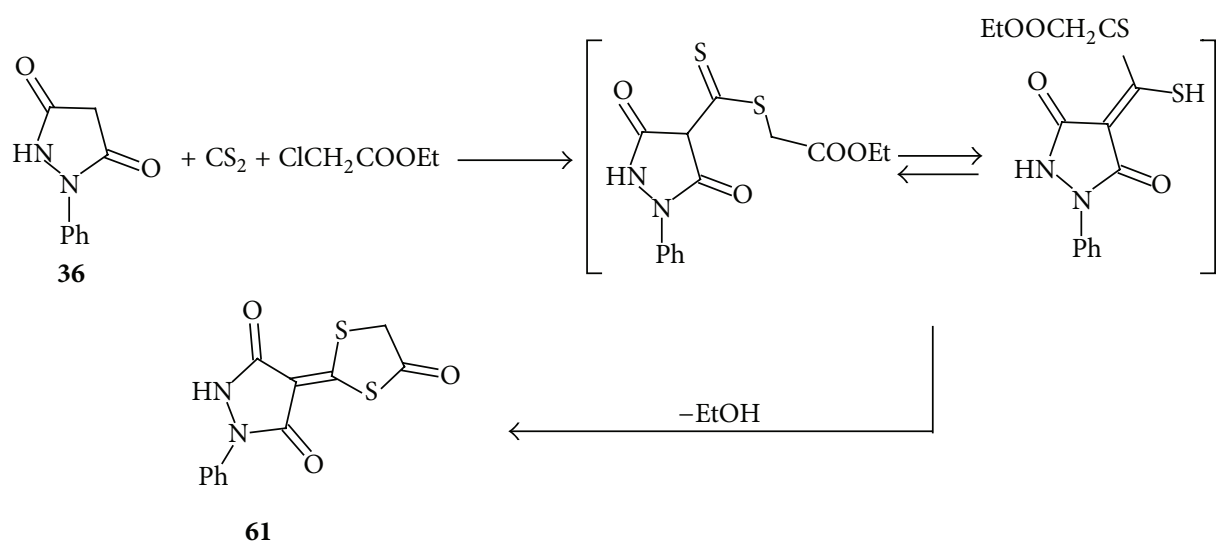

FIgURE 25

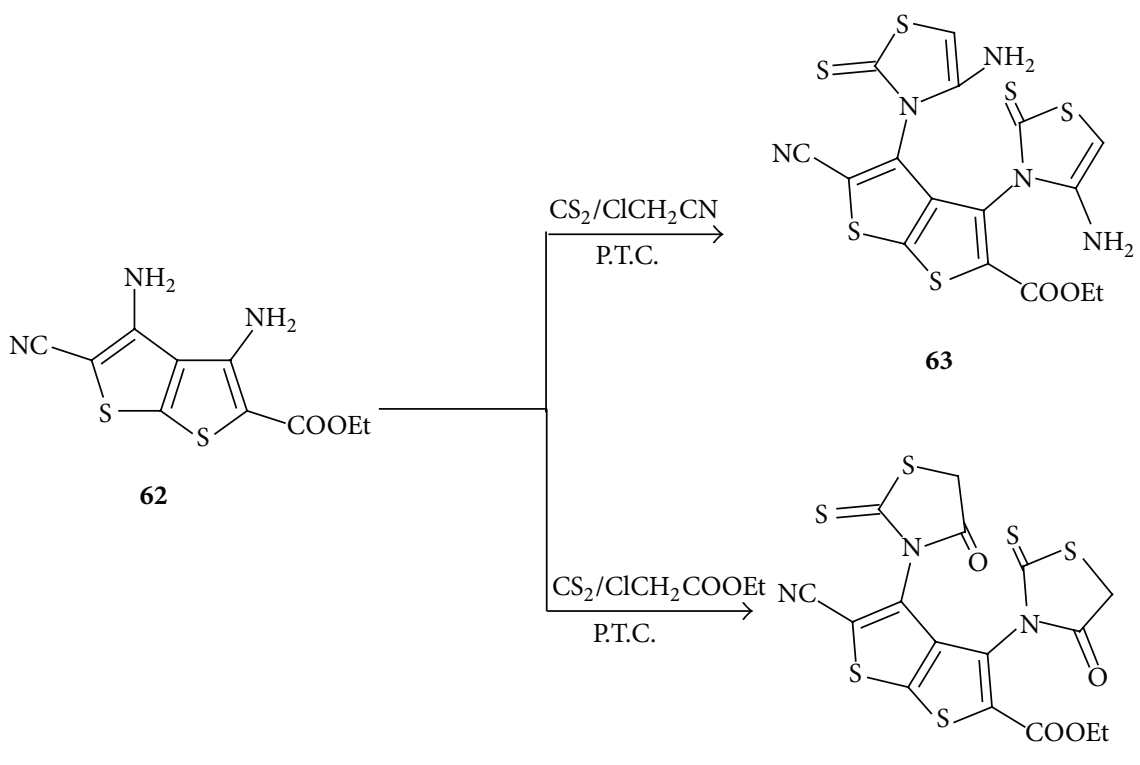

64

Figure 26 
<smiles>CCOC(=O)c1sc2sc(C#N)c(N)c2c1N</smiles>

62

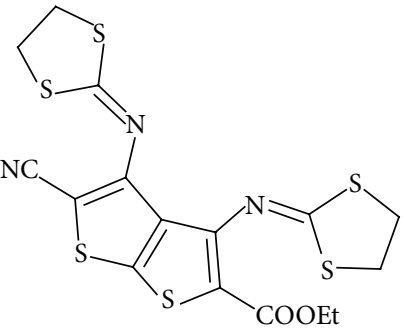

65

FIGURE 27<smiles>CN1N=C(c2ccccc2)CC(=O)NC1=S</smiles>

66

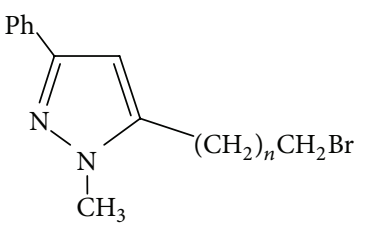

$\begin{aligned} \text { 67a, b } \begin{array}{l}\text { a, } n=0, \\ \text { b, } n=1\end{array} & \end{aligned}$

FIgURE 28

$$
\mathrm{X}-\mathrm{CH}_{2}-\mathrm{N}-\mathrm{NHAr} \longrightarrow \stackrel{+}{\left[\mathrm{CH}_{2}=\mathrm{N}-\mathrm{N}\right]-\mathrm{Ar}} \stackrel{\mathrm{R}-\mathrm{CH}=\mathrm{CH}_{2}}{\mathbf{6 9}}
$$

68

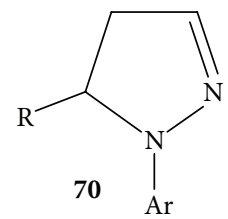

Figure 29
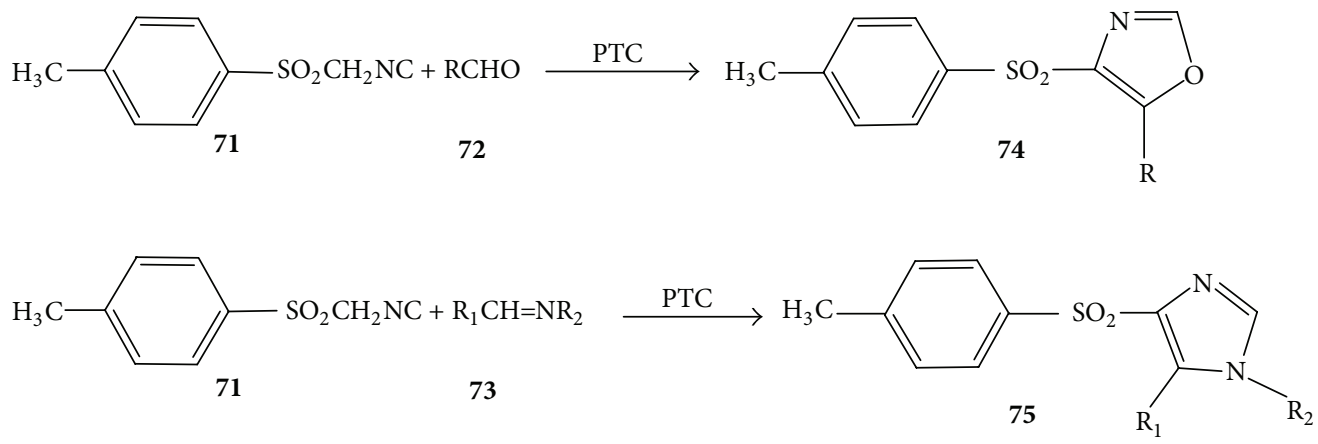

FIgURe 30

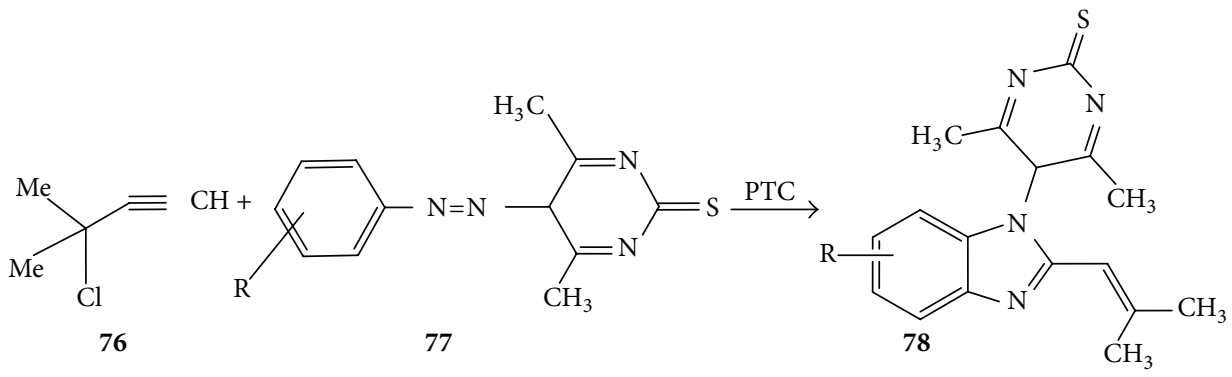

FIGURE 31 


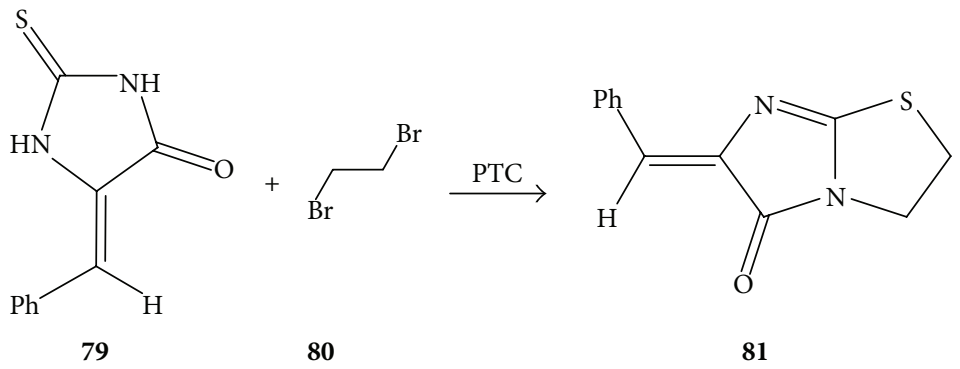

FIGURE 32

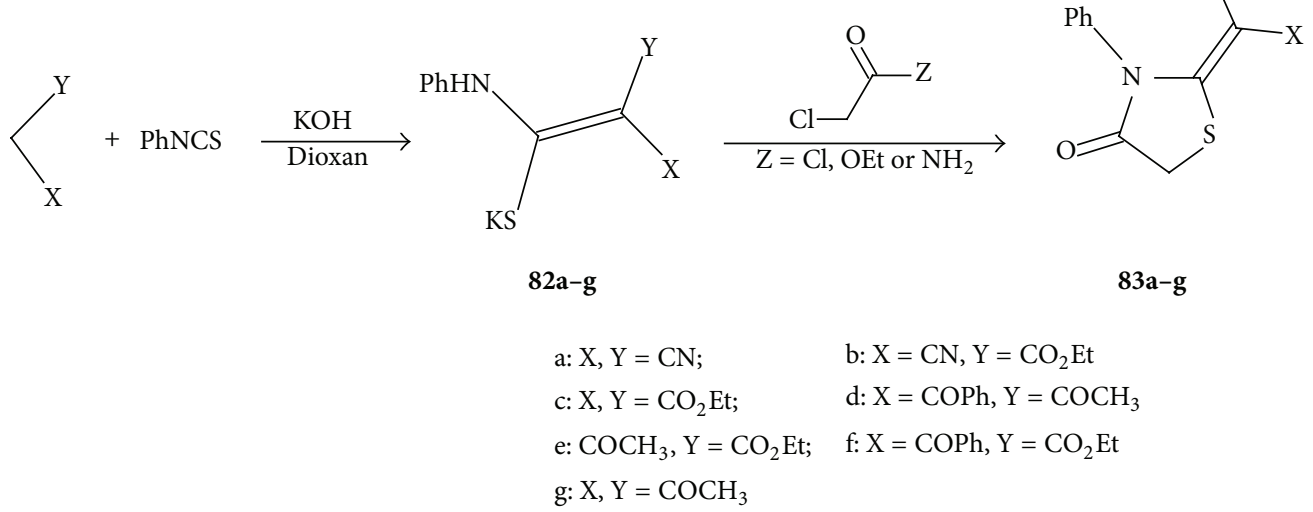

FIgURe 33

(benzene $/ \mathrm{K}_{2} \mathrm{CO}_{3} / \mathrm{TBAB}$ ) to give the corresponding (4Z)-4(4-oxo-1,3-dithiolan-2-ylidene)-1-phenylpyrazolidine-3,5dione 61 [25] (Figure 25).

The addition reaction of ethyl 3,4-diamino-5-cyanothieno[2,3-b]thiophene-2-carboxylate $\mathbf{6 2}$ to carbon disulfide, followed by cyclization reaction through the treatment with chloroacetonitrile or ethyl chloroacetate under PTC conditions $\left(\mathrm{K}_{2} \mathrm{CO}_{3}\right.$, TBAB, dioxane), furnished 3,4bis(4-amino-2-thioxo-1,3-thiazol-3(2H)-yl)-5-propanoylthieno[2,3-b] thiophene-2-carbonitrile 63 and 3,4-bis(4-oxo-2thioxo-1,3-thiazolidin-3-yl)-5-propanoylthieno[2,3-b] thiophene-2-carbonitrile 64, in satisfactory yields [32] (Figure 26).

Moreover, the addition reaction of compound 62 to carbon disulfide followed by cycloalkylation reaction with 1,2-dibromoethane, at 1:1:1 molar ratio under same PTC conditions, afforded the corresponding bis-1,3-dithiolan-2ylidene 65 [32] (Figure 27).

Treatment of triazepene compound $\mathbf{6 6}$ with dibromides using liquid-liquid condition (benzene/ $\mathrm{NaOH} / \mathrm{TBAB}$ ) furnished pyrazole derivatives 67a, b [33] (Figure 28).

1,3-Dipolar cycloaddition of various substituted nitrilimines 68 to the appropriate alkenyl dipolarophiles 69 in aqueous media and in presence of a surfactant afforded a number of 1-aryl-5-substituted-4,5-dihydropyrazoles 70 [34] (Figure 29).

Preparation of 5-substituted oxazoles 74 or imidazoles 75 was achieved by reaction of p-tolylsulphonylmethyl isocyanide 71 with aldehydes $72 \mathrm{R}_{1} \mathrm{CH}=\mathrm{NR}_{2}$ or $73\left(\mathrm{R}_{1}, \mathrm{R}_{2}=\right.$ substituted phenyl, heteroaryl, and alkyl) under liquid-liquid condition [35, 36] (Figure 30).

The interaction of 3-chloro-3-methyl-1-butyne 76 with 4,6-dimethyl-5-arylazo-2-thiopyrimidine 77 under liquid-liquid phase (benzene/aq. $\mathrm{KOH} / \mathrm{BTEACl}$ ) afforded 4,6dimethyl-5-[2-(2-methylprop-1-enyl)-1H-benzimidazol-1-yl] pyrimidinyl-(5H)-thiones 78 [37] (Figure 31).

Reaction of 5-phenylmethylene thiohydantoin 79 with 1,2-dibromoethane $\mathbf{8 0}$ under liquid-liquid conditions (benzene/aq. NaOH/TBAB) afforded 2,3-dihydro-6-phenylmethylene-5-oxo-imidazo[2,3-b] thiazole 81 [38] (Figure 32).

A variety of 4-thiazolidinone derivatives $83 \mathbf{a}-\mathbf{g}$ were successfully synthesized via in situ formation of keteneN,S-acetals 82a-g which in turn was reacted with ethyl chloroethyl acetate, chloroacetamide, or chloroacetyl chloride followed by ring closure to afford the desired 4thiazolidinones 83a-g [39] (Figure 33).

One of the medicinal applications for PTC techniques is synthesis of sibenadet hydrochloride $\mathbf{8 7}$ which is a potent drug used for treatment of chronic obstructive pulmonary disease. This bioactive molecule was synthesized by $\mathrm{O}$ alkylation of phenylethanol $\mathbf{8 4}$ with the alkyl bromide $\mathbf{8 5}$ under PTC condition to form the alkylated product 86 in $97 \%$ yield. Reaction of $\mathbf{8 6}$ with benzothiazole derivative led to formation of the desired product 86 [40] (Figure 34).

Treatment of an equimolar amount of 2-mercaptoquinazolin-4(3ㅂ) -one $\mathbf{8 8}$ and dihalocompounds such as 1,2dibromoethane and chloroacetyl chloride underwent $\mathrm{S}$ - and 


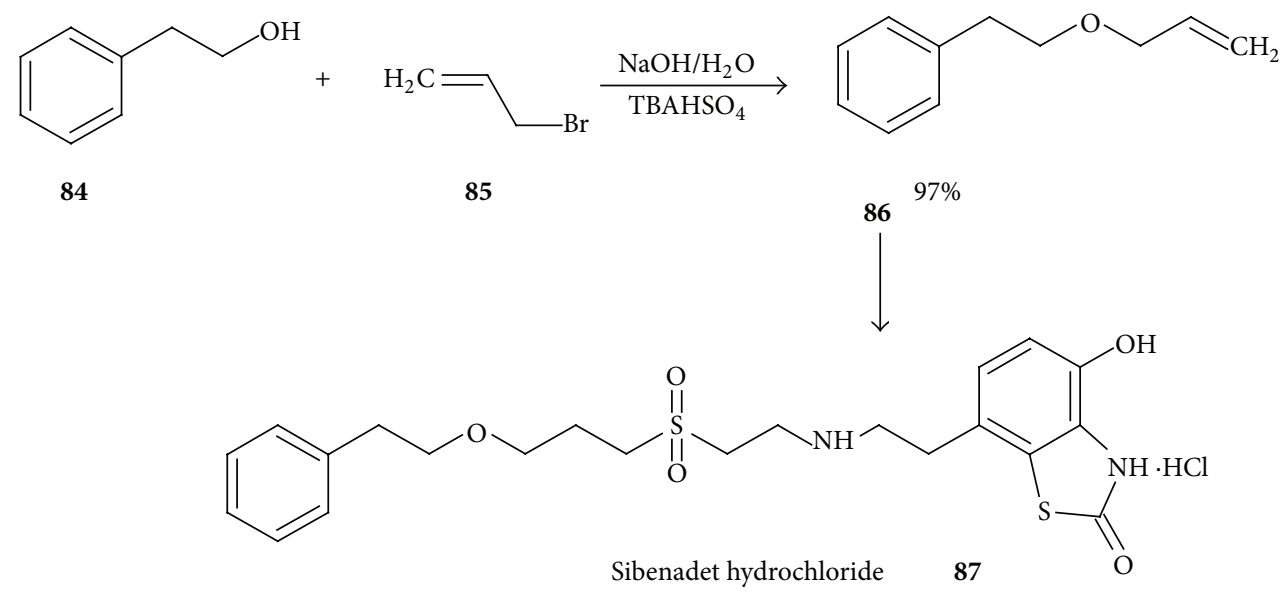

FIgURE 34

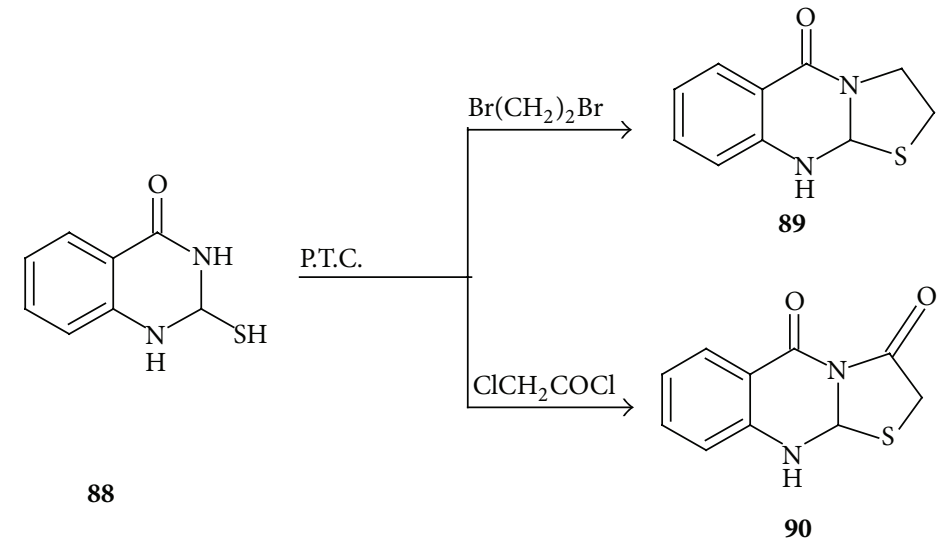

Figure 35

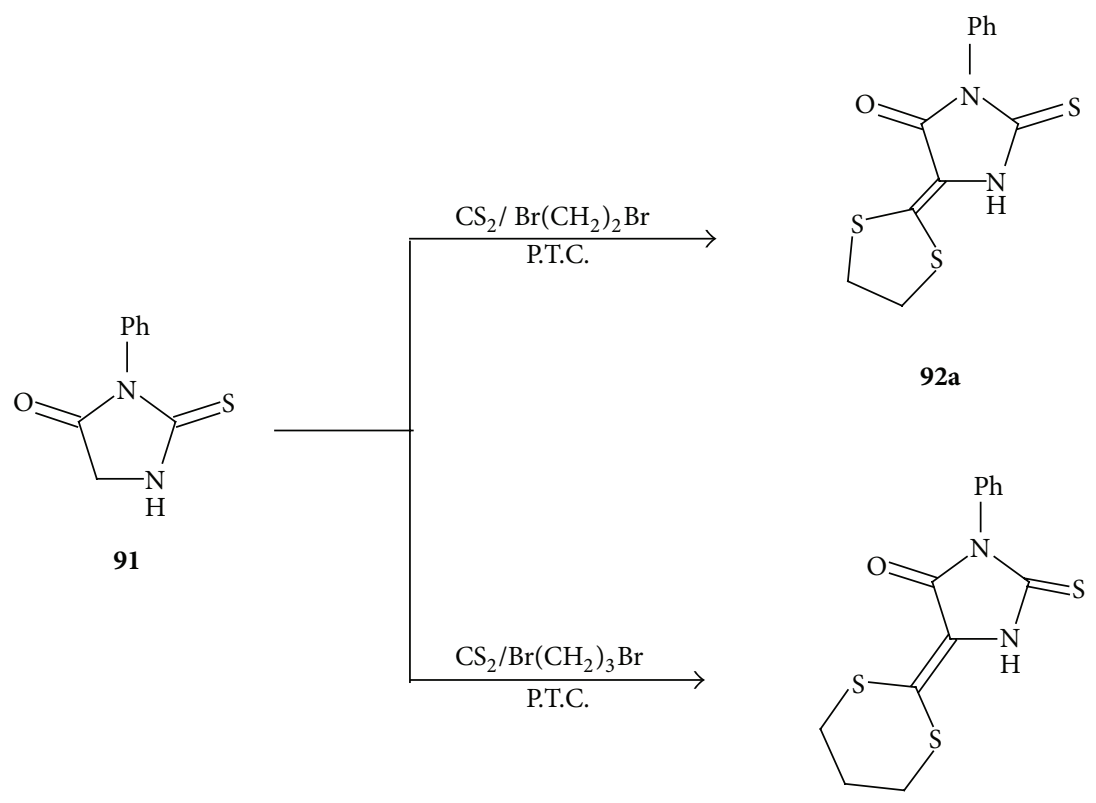

92b

Figure 36 
<smiles>Cc1c(NC(=S)Nc2ccccc2)c(=O)n(-c2ccccc2)n1C</smiles>

$$
93 \quad \mathrm{X}=\mathrm{S}
$$

$94 \quad \mathrm{X}=\mathrm{O}$

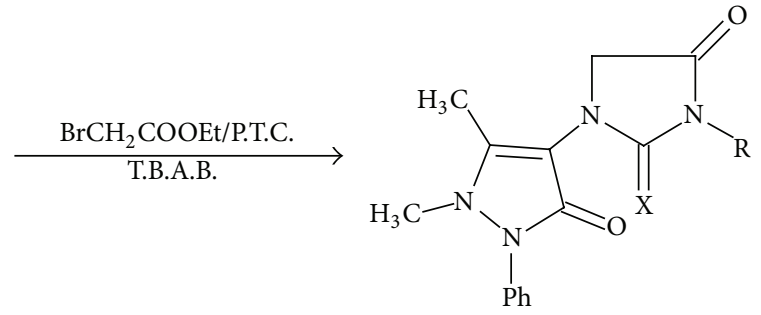

$95 a, b$

FIgURE 37

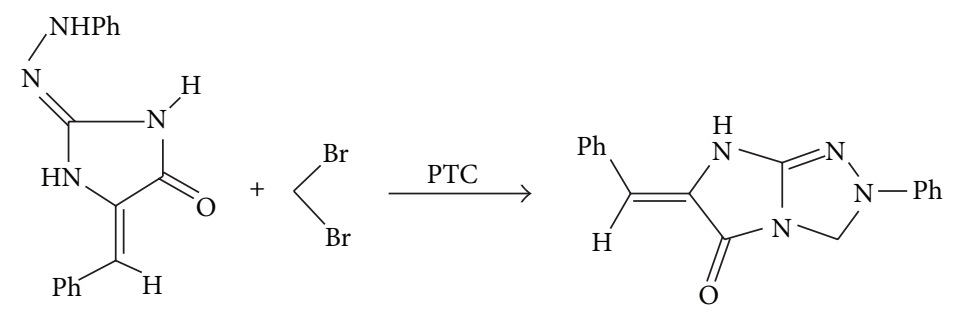

96<smiles>O=C1N/C(=C\c2ccccc2)N/C1=N\O</smiles>

98 99a<smiles>CC(C)CC1=NCON2CON=C1N/C2=C\c1ccccc1</smiles>

97

Figure 38<smiles>Cc1cc(CC#N)cc(CC#N)c1</smiles>

100<smiles>CC(C)(C)c1cc(Cn2cnnc2)cc(C(C)(C)C#N)c1</smiles>

101

Anastrozole

Figure 39

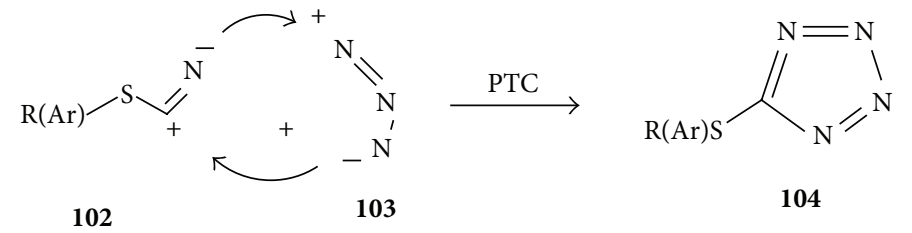

FIGURE 40 
<smiles>[R]c1ccc(/N=C(\Cl)c2cc(/C(Cl)=N/c3ccc([R])cc3)cc(/C(Cl)=N/c3ccc([R])cc3)c2)cc1</smiles>

105

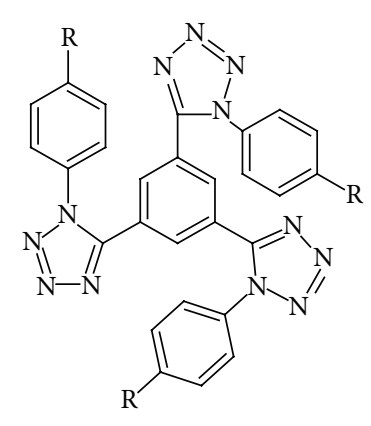

106a-e

$\mathrm{R}=\mathrm{OMe}(\mathrm{a}), \mathrm{OEt}(\mathrm{b}), \mathrm{Me}(\mathrm{c}), \mathrm{H}$ (d), Br (e)

FIGURE 41
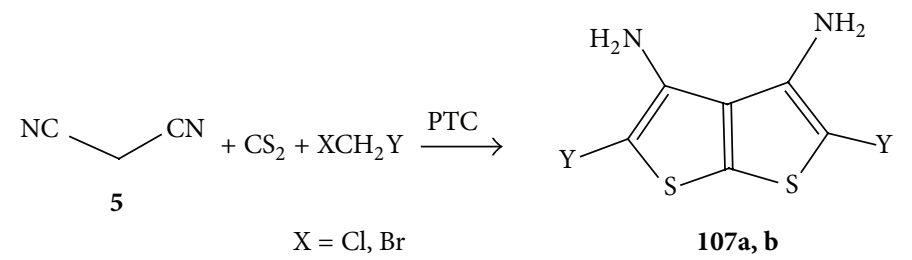

$$
\begin{aligned}
& \mathrm{a}, \mathrm{Y}=\mathrm{COOEt} \\
& \mathrm{b}, \mathrm{Y}=\mathrm{CN}
\end{aligned}
$$

Figure 42

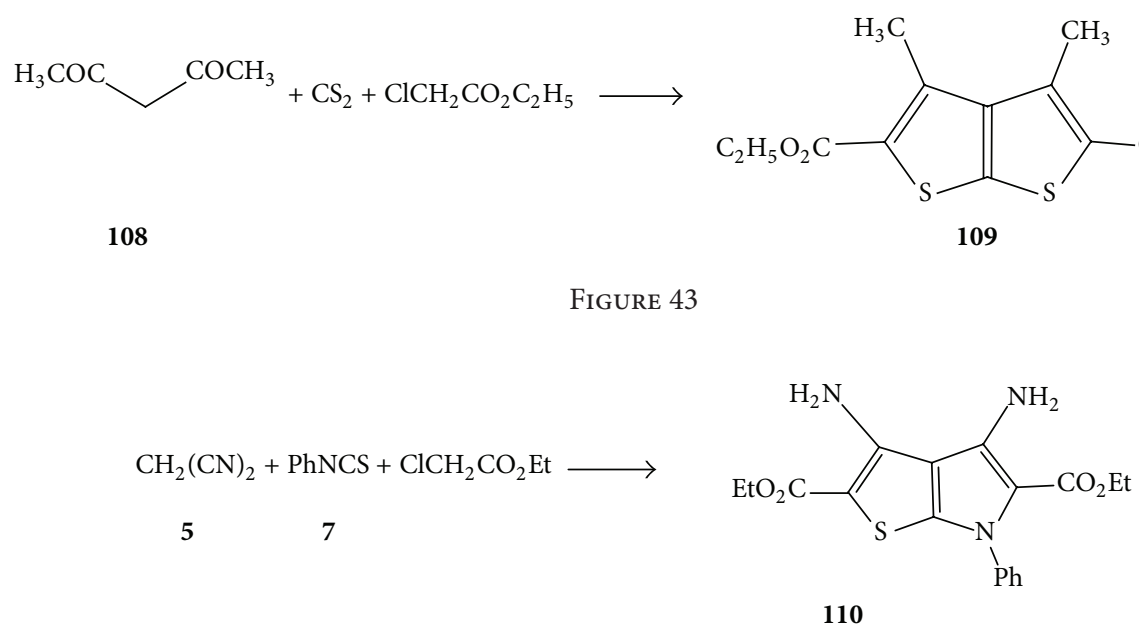

FIgURe 44<smiles>CC1=NN(c2ccccc2)C(=O)C1</smiles><smiles>CC1=NN(c2ccccc2)C(=O)C1C(=S)S</smiles><smiles>CCCCC</smiles><smiles>Cc1nn(-c2ccccc2)c2c1C(=S)SC2C#N</smiles>

FIGURE 45 


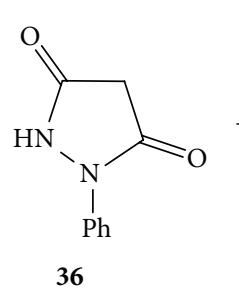

$+\mathrm{CS}_{2}+\mathrm{ClCH}_{2} \mathrm{R}$
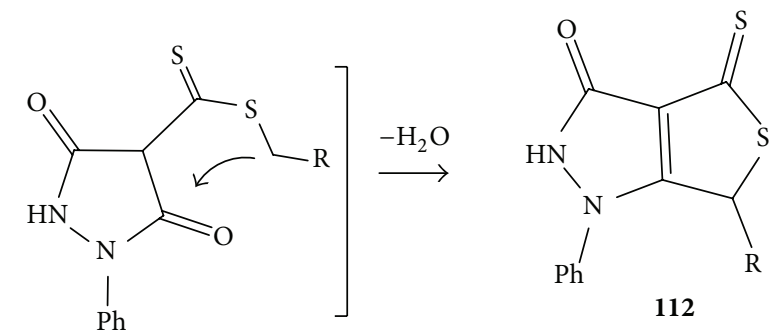

$\mathrm{R}=\mathrm{CN}, \mathrm{COOEt}$

Figure 46<smiles>C=CCBr</smiles>

FIGURE 47

N-cyclo-alkylation by using PTC conditions, giving 2,3-dihydro-5 $\mathrm{H}$ - $[1,3]$ thiazolo[2,3-b] quinazolin-5-one 89 and $5 \underline{\mathrm{H}-}$ [1,3]-thiazolo-[2,3-b]quinazoline-3,5(2프)-dione 90, respectively [41] (Figure 35).

The PTC reaction of 3-phenyl-2-thiohydantoin 91 with dihalocompounds, namely, ethylene dibromide or 1,3-dibromopropane, with $\mathrm{CS}_{2}$, yielded 1,3-dithioxane derivative 92a or 5-(1,3-dithian-2-ylidene)-3-phenyl-2-thioxoimidazolidin4-one derivative 92b, respectively [42] (Figure 36).

Treatment of the thiourea 93 and urea 94 derivatives with ethyl bromoacetate under PTC condition using TBAB as a catalyst and benzene/anhydrous $\mathrm{K}_{2} \mathrm{CO}_{3}$ as liquid-solid phases gave imidazolidinediones $95 \mathbf{a}$, b via ring closure pathway [43] (Figure 37).

\subsection{Synthesis of Five-Membered Ring Heterocycles Containing} Three Heteroatoms. Alkylation of (2Z,5Z)-5-benzylidene2-(hydroxyimino)imidazolidin-4-one 96 with methylene bromide in benzene/aq. $\mathrm{NaOH}$ in presence of TBAB as a catalyst gave 2-phenyl-2,3-dihydro-6-phenylmethylene-5oxo-imidazo[2,1-c]-1,2,4-triazole 97. On the other hand, alkylation of the oxime derivative of 5-phenylmethylene thiohydantoin 98 with methylene bromide gave 3-(1H)-6phenylmethylene-5-oxo-imidazo[2,1-c]-1,2,4-oxadiazoles 99a, b [38] (Figure 38).

Anastrozole 101 which acts as selective aromatase inhibitor and is employed effectively in treatment of advanced breast cancer in postmenopausal women has been synthesized in good yield by methylation reaction of 3,5bis(cyanomethyl)toluene 100, using methyl chloride and 50\% aq. $\mathrm{NaOH} / \mathrm{TEBA}$ as a PTC condition [40] (Figure 39).

2.4. Synthesis of Five-Membered Ring Heterocycles Containing Four Heteroatoms. 5-Alkyl and 5-arylthiotetrazoles 104 were synthesized in good yield from reaction of alkyl(aryl)thiocyanates $\mathbf{1 0 2}$ with azide $\mathbf{1 0 3}$ under PTC conditions [44] (Figure 40).

Functionally substituted tetrazoles have been synthesized from the corresponding $\mathrm{N}, \mathrm{N}^{\prime}, \mathrm{N}^{\prime \prime}$-triarylbenzene-1,3,5tricarboxamides 105 via sequential transformation of these compounds into imidoyl chlorides and treatment of the latter with sodium azide under conditions of phase-transfer catalysis. As a result, a number of heterocyclic structures 106a-106e containing three tetrazole rings have been isolated [45] (Figure 41).

\section{Synthesis of Fused Five-Membered Ring Heterocycles}

Functionally substituted thieno(2,3-b)thiophenes $\mathbf{1 0 7 a}, \mathbf{b}$ were synthesized in a one-pot reaction of malononitrile 5 , $\mathrm{CS}_{2}$, and $\alpha$-halocarbethoxy or $\alpha$-halonitrile electrophiles in 1:1:2 molar ratios using (benzene/ $\mathrm{K}_{2} \mathrm{CO}_{3} / \mathrm{TBAB}$ ) as a PTC condition [23] (Figure 42).

Another different thienothiophene 109 was synthesized via the reaction of acetylacetone $\mathbf{1 0 8}$ with $\mathrm{CS}_{2}$ and ethyl chloroacetate in 1:1:2 molar ratio under the same experimental conditions [46] (Figure 43).

Thieno(2,3-b)pyrrole derivative $\mathbf{1 1 0}$ was obtained by treating malononitrile 5 with phenyl isothiocyante 7 and ethyl chloroacetate in 1:1:2 molar ratio using (benzene/ $\mathrm{K}_{2} \mathrm{CO}_{3} / \mathrm{TBAB}$ ) as a phase transfer catalyst [23] (Figure 44).

Likewise, under the same conditions, 3-methyl-1-phenyl2-pyrazoline-5-one $\mathbf{6 3}$ was subjected to react with $\mathrm{CS}_{2}$ and chloroacetonitrile in 1:1:1 molar ratio where 6-cyano-4,6dihydro-3-methyl-1-phenylthieno(3,4-c)pyrazol-4-thione 111 was obtained in good yield [23] (Figure 45).

1-Phenyl-3,5-pyrazolinedione 36 was allowed to react with $\mathrm{CS}_{2}$ along with chloroacetonitrile or ethyl chloroacetate 


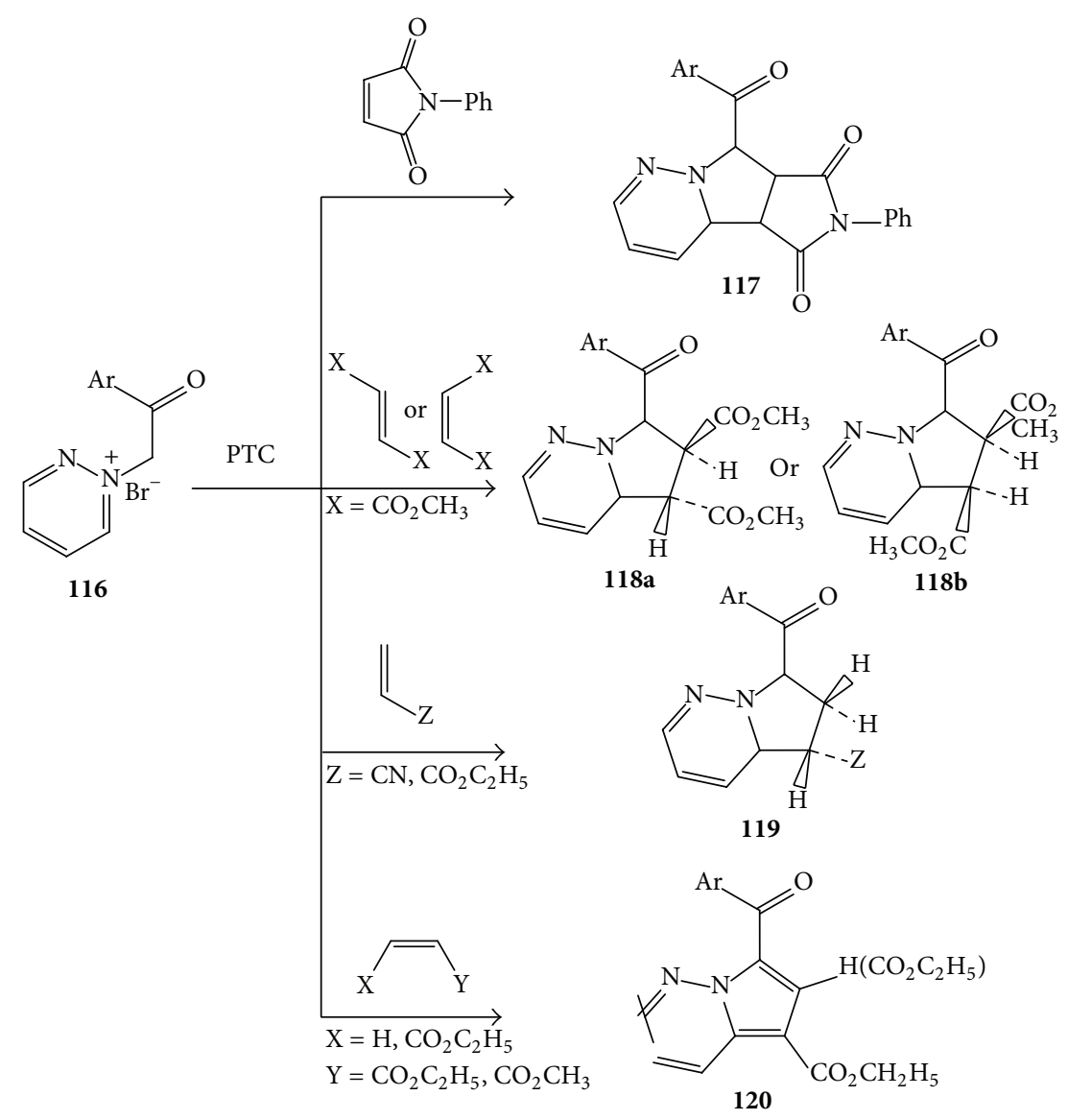

FIgURE 48<smiles>N#Cc1c(S)nc2nc(S)c(C#N)c(N)c2c1N</smiles>

121<smiles>N#Cc1c(Cl)nc2nc(Cl)c(C#N)c(N)c2c1N</smiles>

123<smiles></smiles>

122 $\mathrm{Y}=\mathrm{COOEt}, \mathrm{CN}, \mathrm{PhCO}, \mathrm{CONH}_{2}$ PTC

Figure 49

under the same conditions to give the corresponding thioxothienopyrazolone derivatives 112 [25] (Figure 46).

Reaction of 4-hydroxydithiocoumarin 113 with allyl bromide 114 under liquid-liquid technique $\left(\mathrm{CH}_{2} \mathrm{Cl}_{2} / \mathrm{aq}\right.$. $\mathrm{NaOH} / \mathrm{TBAB}$ or TBACl) gave 2-methyl-2,3-dihydro- $4 \mathrm{H}$ thieno[2,3-b]benzothiopyran-4-one 115 [47] (Figure 47).

Treating of pyridazinium ylides $\mathbf{1 1 6}$ with $\mathrm{N}$-phenylmaleimide, maleic and fumaric esters, resulted in the cycloadduct products $\mathbf{1 1 7 - 1 2 0}$ with high stereospecificity in the presence of KF and trioctylmethylammonium chloride or without solvent in the presence of aliquat $\mathbf{3 3 6}$ as phase transfer catalyst [48] (Figure 48).

Under solid-liquid technique (dioxan $/ \mathrm{K}_{2} \mathrm{CO}_{3} / \mathrm{TBAB}$ ), bis-thieno $(1,8)$ naphthyridines $\mathbf{1 2 2}$ were prepared by reaction of 4,5-diamino-3,6-dicyano-(1,8)naphthyridine-2,7dithiol 121 with ethyl chloroacetate, chloroacetonitrile, 


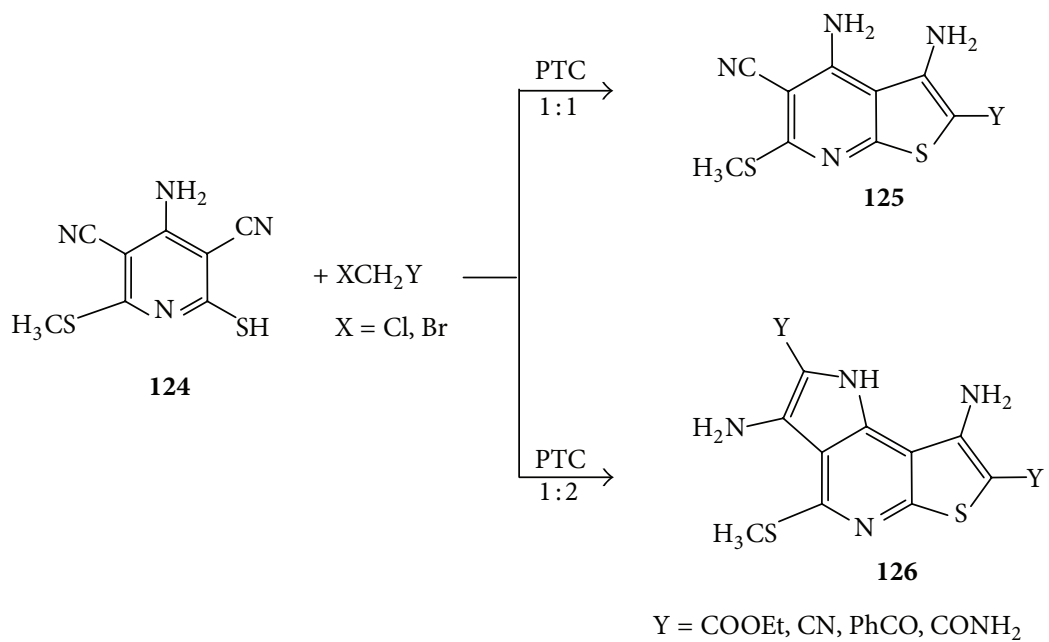

FIGURE 50

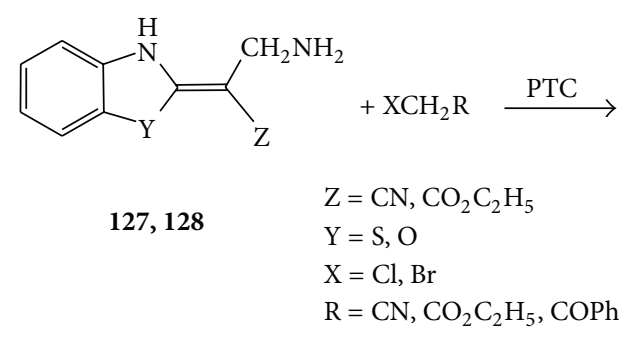<smiles>[Z]c1c(N)c([R])n2c1[Y]c1ccccc12</smiles>

129,130

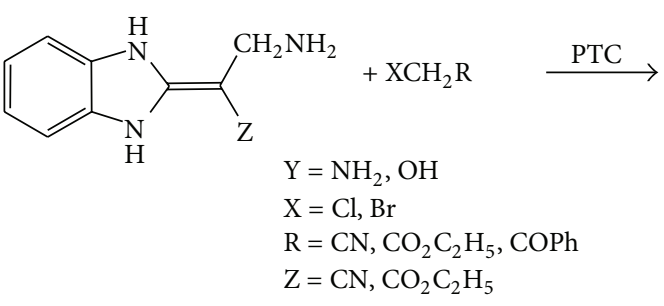<smiles>[Y]c1c([R])n2c3ccccc3n3c([R])c(N)c1c23</smiles>

$131 \mathrm{a}, \mathrm{b}$

Figure 51

phenacyl bromide, or chloroacetamide in 1:2 molar ratio or from reaction of 4,5-diamino-2,7-dichloro-3,6-dicyano(1,8)naphthyridine $\mathbf{1 2 3}$ with two equivalents of ethyl mercaptoacetate [49] (Figure 49).

Similarly 4-amino-3,5-dicyano-2-mercapto-6-methylthiopyridine 124 was reacted with ethyl chloroacetate, chloroacetonitrile, phenacyl bromide, or chloroacetamide in 1:1 molar ratio using the same PTC condition to give the corresponding thieno(2,3-b)pyridines $\mathbf{1 2 5}$ or pyrrolo(2,3-d) thieno(2,3-b)pyridines 126 [49] (Figure 50).

Using solid-liquid technique (dioxan $/ \mathrm{K}_{2} \mathrm{CO}_{3} / \mathrm{TBAB}$ ), 2-ylidenemalononitrile and ethyl 2-ylidenecyanoacetate of both 2,3-dihydrobenzothiazole 127 and 2,3-dihydrobenzoxazole 128 were allowed to react with chloroacetonitrile, ethyl chloroacetate, or phenacyl bromide in equimolar ratio which afforded the corresponding substituted pyr-rolo[2,1b]benzothiazoles $\mathbf{1 2 9}$ or substituted pyrrolo[2,1-b]benzoxazoles 130, respectively [42]. Similarly, (3-amino-2-(1,3dihydro-2H-benzimidazol-2-ylidene)propanenitrile malononitrile 131a or ethyl 3-amino-2-(1,3-dihydro-2H-benzimidazol-2-ylidene)propanoate $\mathbf{1 3 1 b}$ were treated under the same PTC conditions, where the corresponding pyrrolo[2,3b]pyrrolo[1,2-f]benzoimidazoles 132 were obtained [50] (Figure 51).

Reaction of 2,7-dichloro-1,8-naphthyridine derivative $\mathbf{1 3 3}$ with ethyl glycinate hydrochloride in 1:1 molar ratio under solid-liquid technique (dioxan $/ \mathrm{K}_{2} \mathrm{CO}_{3} / \mathrm{TBAB}$ ) afforded the corresponding bis-pyrrolo $(1,8)$ naphthyridine derivative 134 [49] (Figure 52). 


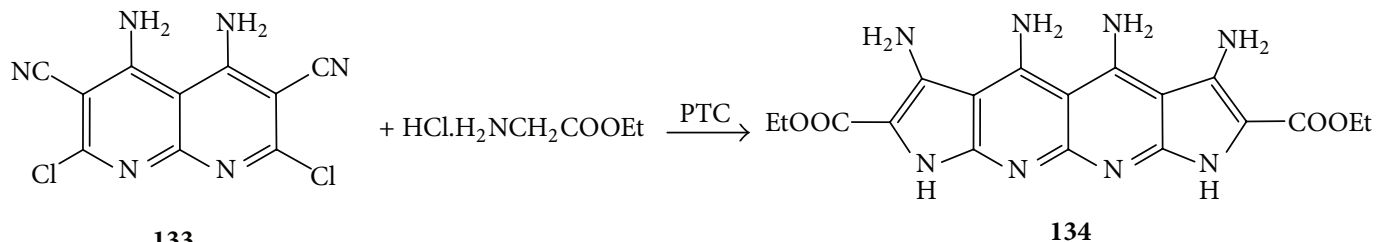

133

FIgURE 52<smiles>[Z]c1c(-c2ccccc2)cc(=N)sc1S</smiles>

$\underset{1: 1}{\stackrel{\mathrm{PTC}}{\longrightarrow}}$<smiles>[Y]c1c(C(=O)OCC)sc2sc(=N)c(C#N)c(-c3ccccc3)c12</smiles>

135

CN, $\mathrm{CHO}$ PhCO
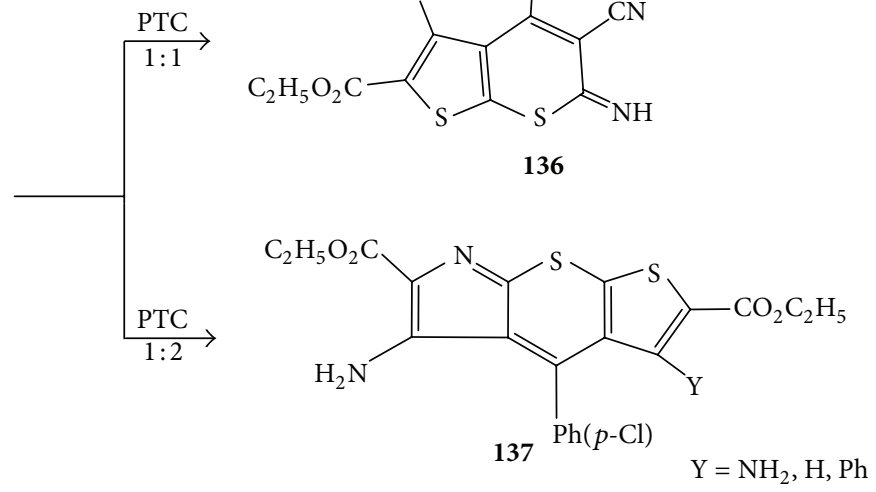

Figure 53<smiles>NC1=NN(c2ccccc2)C(=O)C1</smiles>

138<smiles>N#CC1=C2C(C(=S)S1)C(N)=NN2c1ccccc1</smiles>

$\mathrm{Ph}$<smiles>CCOC(=O)C1=C2C(C(=S)NN2C)C(N)=NN1</smiles>

$\mathrm{Ph}$

$\mathrm{PhCOCH}_{2} \mathrm{~B}$ $P$ $(1: 1: 1) / \mathrm{PTC}$<smiles>[Y11]C1=NN(c2ccccc2)C2=C(C(=O)c3ccccc3)SC(=S)C12</smiles>
$\stackrel{\mathrm{ClCH}_{2} \mathrm{CO}_{2} \mathrm{Et}}{\stackrel{(1: 1: 2) / \mathrm{PTC}}{\longrightarrow}}$<smiles>CCOC(=O)c1sc(CC(=O)O)c2c1c(N)nn2-c1ccccc1</smiles>

$\mathrm{Ph}$<smiles>NNc1ccccc1</smiles><smiles>CCOC(=O)c1cc2c(s1)SCC(=O)NC2</smiles>

143

144

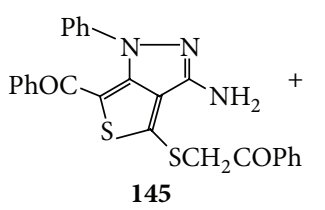<smiles></smiles>

146

$\underset{(1: 1: 2) / \mathrm{PTC}}{\stackrel{\mathrm{ClCH}_{2} \mathrm{CN}}{\longrightarrow}}$

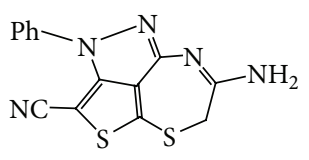

147

FIgURE 54 
<smiles>CCOC(=O)/C(C#N)=C1\SC(=S)C(C#N)=C1N</smiles>

$31 b$<smiles>CCOC(=O)/C(C#N)=C1\SC(=S)c2c1[nH]c(C(=O)c1ccccc1)c2N</smiles>

Figure 55<smiles>[X]C1=C(S)N(c2ccccc2)C(=C(C#N)C#N)C=C1N</smiles>
a; $\mathrm{X}=\mathrm{CN}, \quad \mathbf{b} ; \mathrm{X}=\mathrm{COOEt}$<smiles>[Y]c1c(C(=O)c2ccccc2)sc2c1C(N)=CC(=C(C#N)C#N)N2c1ccccc1</smiles>

150a, b a; $\mathrm{Y}=\mathrm{NH}_{2} \quad$ b; $\mathrm{Y}=\mathrm{OH}$

FigURe 56<smiles>[R]C=Cc1c(C(=O)c2ccccc2)oc2cc3oc(C(=O)c4ccccc4)c(C)c3cc12</smiles>

$\mathrm{R}=4-\mathrm{ClC}_{6} \mathrm{H}_{4}, 4-\mathrm{MeOC}_{6} \mathrm{H}_{4}{ }^{-}$

FIgURE 57<smiles>[R]C=CC(=O)c1cc(C(=O)/C=C/[R])c(O)cc1O</smiles>
153<smiles>[R]C=Cc1oc2cc3oc(C(=O)c4ccccc4)c(C=C[R])c3cc2c1C(=O)c1ccccc1</smiles>

$\mathrm{R}=p-\mathrm{Cl}-\mathrm{C}_{6} \mathrm{H}_{4}, p-\mathrm{MeOC}_{6} \mathrm{H}_{4}$

FIgURE 58

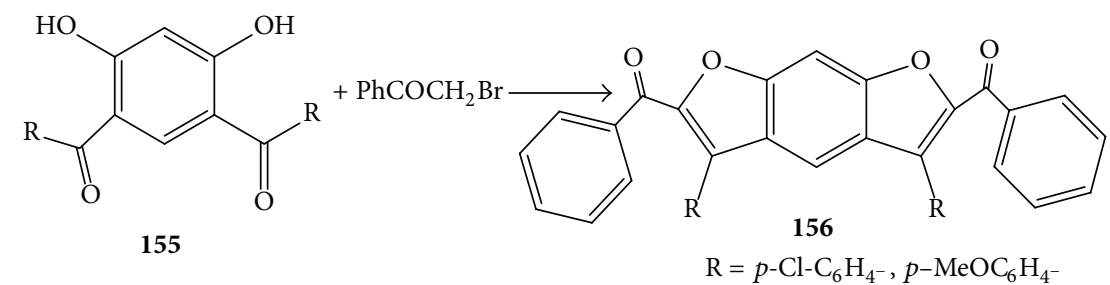

FIGURE 59 
<smiles>[Y17]C(=O)c1cc(C([R14])=O)c(O)cc1O</smiles>

FigURE 60

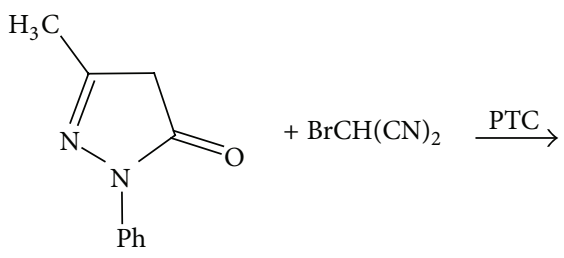

63<smiles>Cc1nn(-c2ccccc2)c2oc(N)c(C#N)c12</smiles>

159

FIgURE 61

Thieno(2,3-b) thiopyran derivatives 136 were obtained from reaction of the thiopyran derivative 135 with ethyl chloroacetate in 1:1 molar ratio (dioxan $/ \mathrm{K}_{2} \mathrm{CO}_{3} / \mathrm{TBAB}$ ), while on carrying out the same reaction under the same conditions but in 1:2 molar ratio gave the corresponding thienopyrrolothiopyran derivatives 137 [51] (Figure 53).

Treatment of 3-amino-1-phenyl-2-pyrazoline-5-one 138 with $\mathrm{CS}_{2}$ along with chloroacetonitrile, ethyl chloroacetate, or phenacyl bromide in $1: 1: 1$ and $1: 1: 2$ molar ratios using [dioxan $/ \mathrm{K}_{2} \mathrm{CO}_{3} / \mathrm{TBAB}$ ] as a PTC gave the corresponding thienopyrazoles 139-143, 145 and respectively, 144, 146 and 147, fused thiazines [52] (Figure 54).

Thieno(3,4-b)pyrrole derivative 148 was obtained by reacting ethyl thiophene-2-ylidenecyanoacetate derivative 31b with phenacyl bromide in a heterogeneous mixture of (dioxan $/ \mathrm{K}_{2} \mathrm{CO}_{3} / \mathrm{TBAB}$ ) as a PTC [19] (Figure 55).

In the same manner, pyridin-2-ylidenemalononitriles 149a, b were reacted with phenacyl bromide under the same reaction condition and yielded the corresponding thieno(2,3b)pyridine derivatives 150a, b [18] (Figure 56).

2,6-Dibenzoyl-5-methyl-3-(substituted styryl)benzo[1,2b:5,4-b]difurans 152 were synthesized from reaction of cinnamoylbenzofurans $\mathbf{1 5 1}$ with phenacyl bromide under liquid-liquid technique (benzene/aq. $\mathrm{K}_{2} \mathrm{CO}_{3} / \mathrm{TBAHSO}_{4}$ ) [53] (Figure 57).

Similarly, 2,6-dibenzoyl-3,5-distyrylbenzo(1,2-b:5,4'-b) difurans 154 were prepared by condensing substituted dichalcones 153 with phenacyl bromide under the same preceding PTC conditions [54] (Figure 58).

Also, under the same PTC conditions, dibenzoylbenzodifurans $\mathbf{1 5 6}$ were obtained from the reaction of substituted resorcinols 155 with phenacyl bromide [55] (Figure 59).

Moreover, 2,6-diaroyl/naphthoyl-3,5-dialkyl/phenylbenzo[1,2-b:5,4- $\left.\mathrm{b}^{\prime}\right]$ difurans 158 were synthesized by condensing 2,4-diacyl/diaroylresorcinols 157 with various p-substituted $\alpha$-bromo ketones [56] (Figure 60).
Treatment of 3-methyl-1-phenyl-2-pyrazolin-5-one 63 with bromomalononitrile in 1:1 molar ratio yielded 5amino-4-cyano-3-methyl-1-phenylfuro(2,3-c)pyrazoline 159 [23] (Figure 61).

Furo(1,5)benzodiazepin derivative $\mathbf{1 6 1}$ was obtained by reaction of 1,4-dimethyl-3H-(1,5)benzodiazepin-2-one 160 with chloroacetonitrile under solid-liquid condition (dioxan $/ \mathrm{K}_{2} \mathrm{CO}_{3} / \mathrm{TBAB}$ ) [57] (Figure 62).

By reaction of 4-methyl-1,3-dihydro-2H-1-benzazepin2-one 162 with chloroacetonitrile or phenacyl bromide under the solid-liquid phase transfer catalyst (dioxan/ $\mathrm{K}_{2} \mathrm{CO}_{3}$ /TBAB), the corresponding pyrrolo[1,2-a](1,5)benzodiazepine 163 was obtained [58] (Figure 63).

Synthesis of 2,6-di(1-acetyl-2-oxopropylidene)dithiolo [4,5-b: $4^{\prime}, 5^{\prime}$-e]-4,8-benzoquinone 166 was achieved in one-pot reaction under solid-liquid technique (benzene/ $\mathrm{K}_{2} \mathrm{CO}_{3} / \mathrm{TBAB}$ ) starting from acetylacetone, $\mathrm{CS}_{2}$ to give $\mathbf{1 6 5}$ which in turn reacted with tetrabromo $p$-benzo-quinone 164 in 2:1 molar ratio [59] (Figure 64).

Treatment of 4-arylidene-1-phenyl-3,5-pyrazolinediones 167 with S,S-acetal derivatives using solid-liquid technique (dioxan $/ \mathrm{K}_{2} \mathrm{CO}_{3} / \mathrm{TBAB}$ ) yielded the corresponding pyrazolino-(1,3)-dithiolane derivatives 168 [25] (Figure 65).

Some condensed heterocyclic systems 171 were obtained by reacting 3-phenyl-4-amino-s-triazole-5-thiol 169 as a dianionic compound containing $\mathrm{N}$ and $\mathrm{S}$ poles with some di- and tetrahaloderivatives $\mathbf{1 7 0}$ as well as $\alpha$-haloketones and $\alpha$-halonitrile derivatives under solid-liquid technique [60] (Figure 66).

2-Aminoprop-1-ene-1,1,3-tricarbonitrile 172 was reacted with $\mathrm{CS}_{2}$ or PhNCS along with 3-methyl-1-phenyl-2-pyrazoline-5-one 63 or 2-iminothiazolidin-4-one 173 (dioxan/ $\mathrm{K}_{2} \mathrm{CO}_{3}$ /TBAB) to give the corresponding fused pyrazoles 174 and thiazoles 175, respectively [61] (Figure 67).

The reaction of 1,3-dihydro-4-methyl $(1,5)$ benzodiazepin2-one $\mathbf{1 7 6}$ with chloroacetonitrile using solid-liquid 


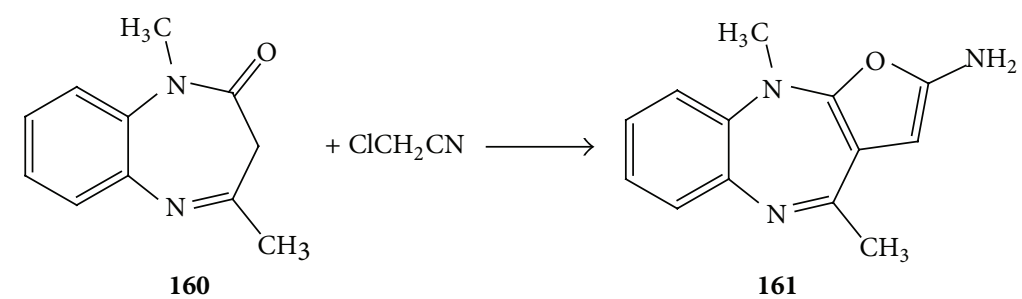

FIgURE 62
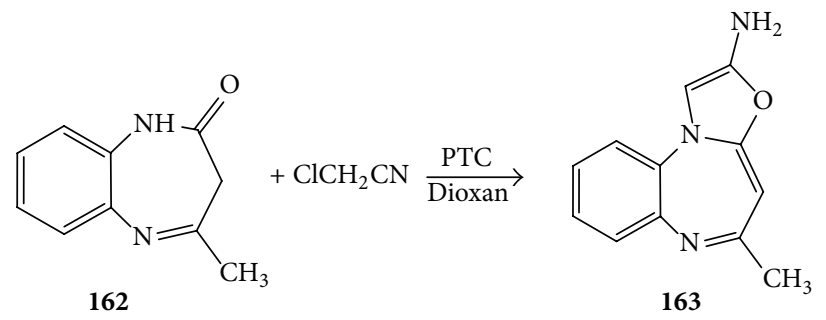

FigURE 63<smiles>CC(=O)C(C(C)=O)=C([As])[As]</smiles><smiles>CC(=O)C(C(C)=O)=c1sc2c(=O)c3sc(=C(C(C)=O)C(C)=O)sc=3c(=O)c=2s1</smiles>

FIGURE 64

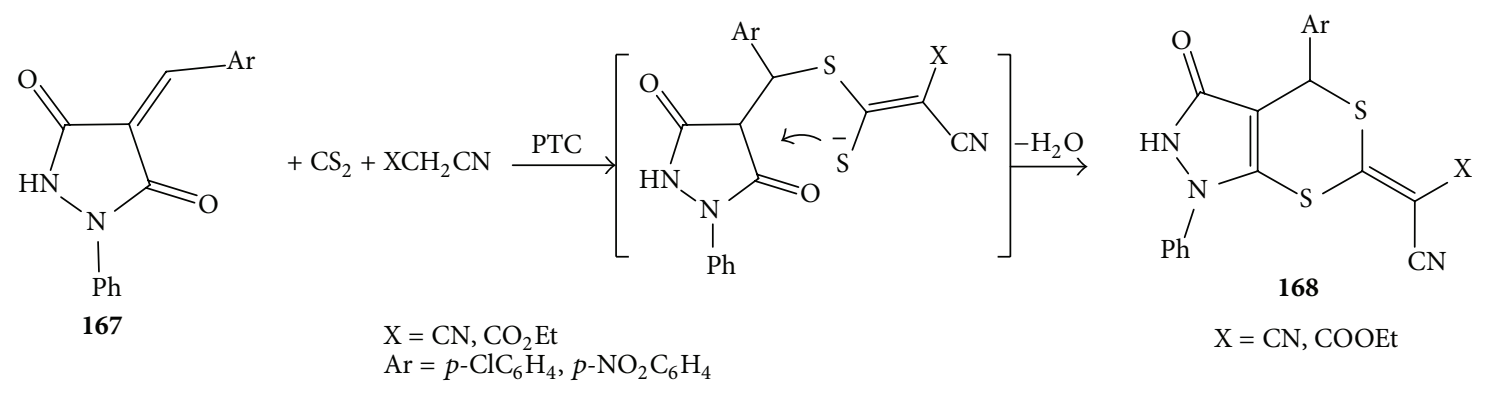

FIGURE 65<smiles>Nn1c(S)nnc1-c1ccccc1</smiles>

169<smiles>CC(C)CC1=C(Br)C(=O)C(Br)=C(Br)C1=O</smiles>

Figure 66 


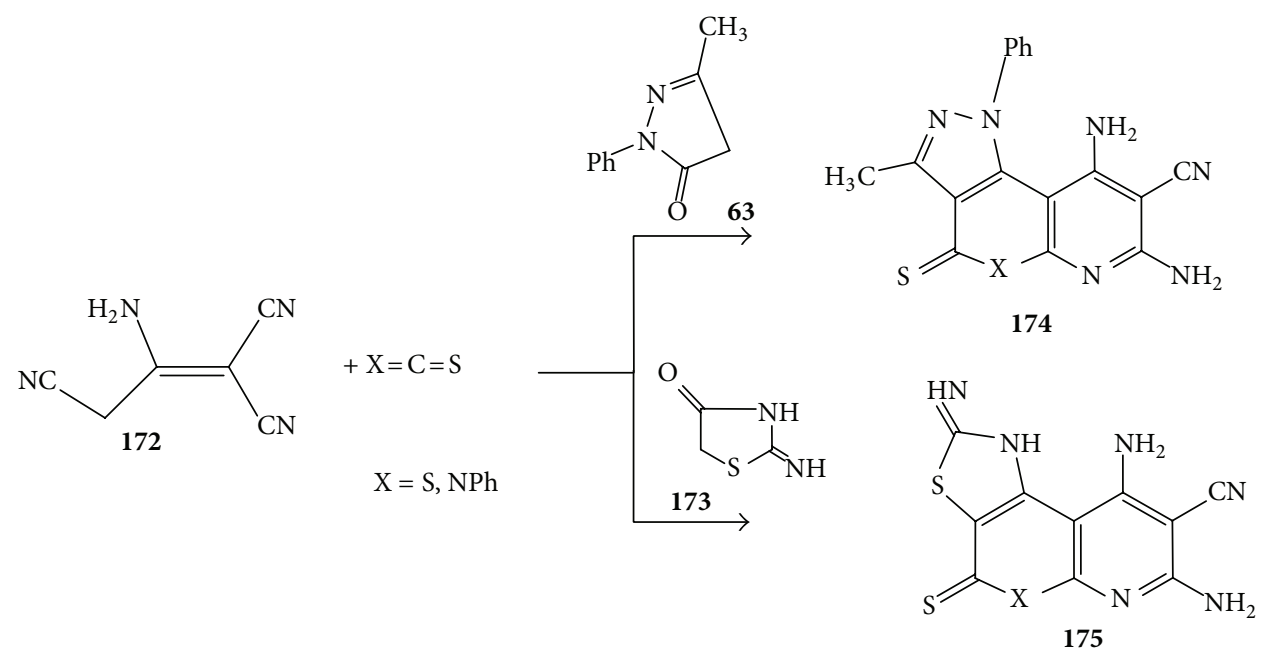

Figure 67<smiles>CC1=Nc2ccccc2NC(=O)C1</smiles>

167<smiles>CC1=Nc2ccccc2N2C=C(N)OC2=C1</smiles>

177

Figure 68<smiles>C[N+]=C1C=Cc2c(C)cc(=O)oc2C1=O</smiles>

178

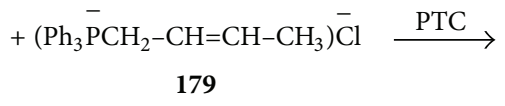

FIGURE 69

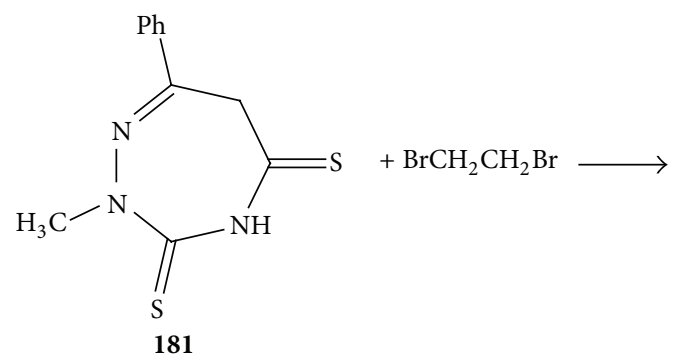

18

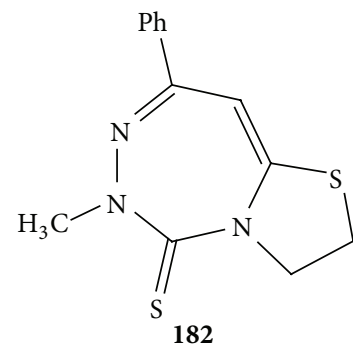

182

FIGURE 70 


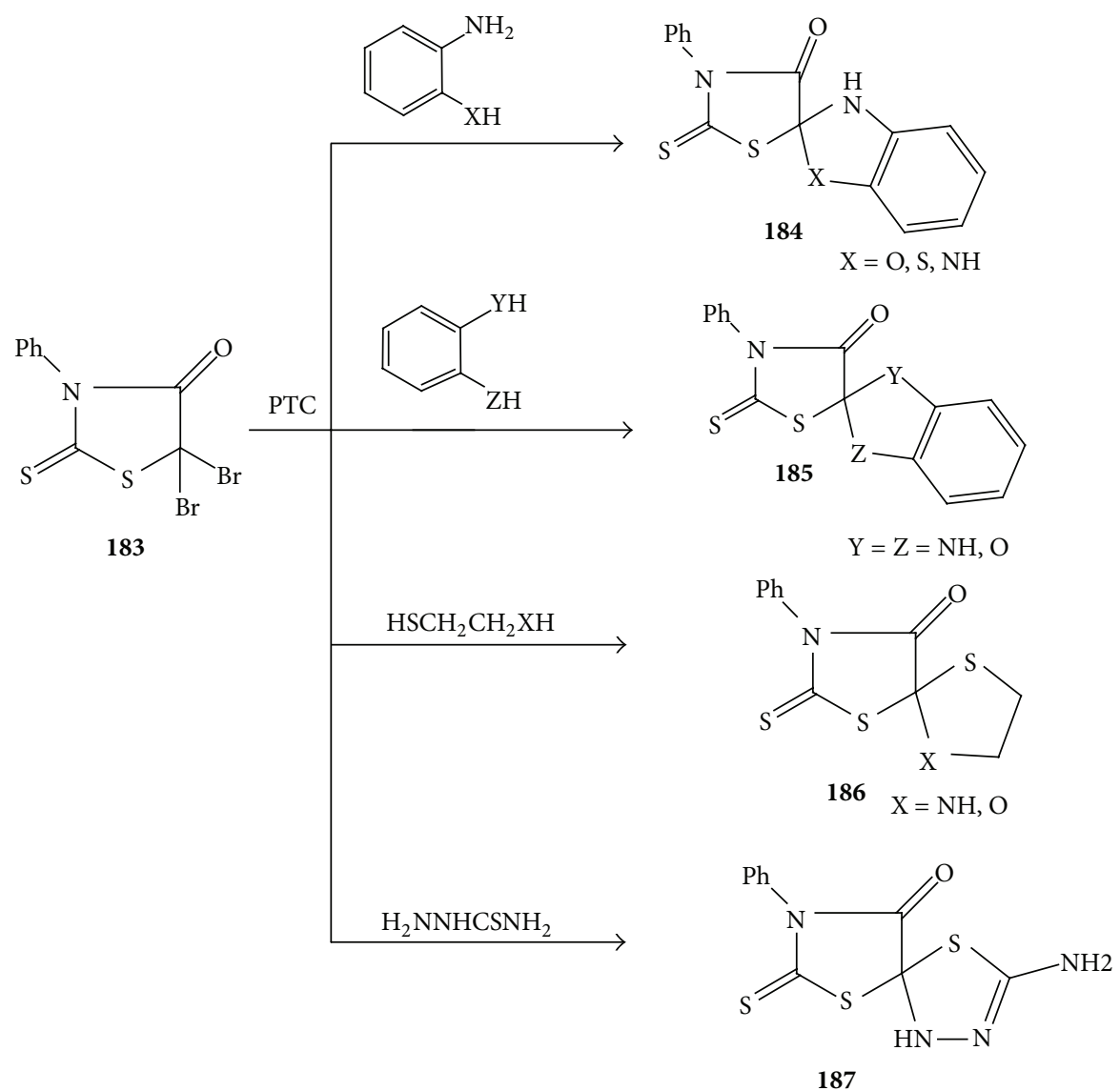

FIGURE 71

technique (dioxan/ $\mathrm{K}_{2} \mathrm{CO}_{3} / \mathrm{TBAB}$ ) gave the corresponding oxazolo-(1,5)benzodiazepin derivative 177 [57] (Figure 68).

When 7-(methoxyimino)-4-methyl-2H-chromene-2,8(7H)-dione $\mathbf{1 7 8}$ was treated with crotyltriphenylphosphonium chloride (CTPPCl) $179\left(\mathrm{CH}_{2} \mathrm{Cl}_{2} / \mathrm{Li}(\mathrm{OH}) / \mathrm{CTPPCl}\right)$, the corresponding chromeno-oxazolone $\mathbf{1 8 0}$ was obtained in which one of the reactants $(\mathrm{CTPPCl})$ acts also as a catalyst [62] (Figure 69).

The triazepine $\mathbf{1 8 1}$ was treated with 1,2-dibromoethane under liquid-liquid technique (benzene/aq. $\mathrm{NaOH} / \mathrm{BTEACl}$ ) to afford the corresponding 6-methyl-8-phenyl-2,3-dihydro[1,3] thiazolo[3,2-d][1,2,4] triazepine-5(6H)-thione 182 [33] (Figure 70).

\section{Synthesis of Spiro Five-Membered Ring Heterocycles}

Synthesis of spirorhodanine heterocycles 184-187 was achieved by treating 5,5-dibromo-3-phenyl-2-thioxo-1,3-thiazolidin-4-one 183 with different bidentates or with mixture of $\mathrm{CS}_{2}$ and some active methylene compounds under solidliquid condition (dioxan/ $\mathrm{K}_{2} \mathrm{CO}_{3} / \mathrm{TBAB}$ ) [63] (Figure 71).

Reaction of 4-ethoxymethylene-1-phenyl-3,5-pyrazolidinedione 188 with $\mathrm{CS}_{2}$ and malononitrile 5 or ethyl cyanoacetate 11 using solid-liquid technique (dioxan/ $\mathrm{K}_{2} \mathrm{CO}_{3} / \mathrm{TBAB}$ ) yielded the corresponding spiro-1,3-dithiolane derivatives 189 [25] (Figure 72).

1-Benzoyl-3,3-dibromo-4-phenyl-(1,5)benzodiazepin2-one 190 was reacted with different dinucleophiles under PTC condition (dioxan $/ \mathrm{K}_{2} \mathrm{CO}_{3} / \mathrm{TBAB}$ ) to furnish the corresponding spiroheterocycles attached to benzodiazepine moiety 191-193 [64] (Figure 73).

\section{Uses of Phase Transfer Catalysis Techniques in Reactions of Five-Membered Heterocycles}

\subsection{Alkylation and Acylation}

5.1.1. N-Alkylation or Acylation. Diez-Barra et al. [65] studied the alkylation of pyrrole 194 by PTC technique in absence of the solvent. The study revealed that the $\mathrm{N}$ versus $\mathrm{C}$ ratio is not affected by the nature of the catalyst. The nature of the leaving groups have a significant influence where $\mathrm{N}$ alkylation increases in the sequence $\mathrm{I}<\mathrm{Br}<\mathrm{Cl}<\mathrm{OTs}$ (Figure 74).

N-alkyl pyrroliden-2,5-diones $200[66,67]$ were synthesized via reaction of pyrroliden-2,5-diones with different alkylating reagents under phase transfer catalysis condition (toluene $/ \mathrm{K}_{2} \mathrm{CO}_{3} / \mathrm{TBAHSO}_{4}$ ) according to (Figure 75 ).

$\mathrm{N}$-allylindoles 203 were easily carried out via $\mathrm{N}$-allylation of the proper indoles 201 with the suitable allyl halides 202. 


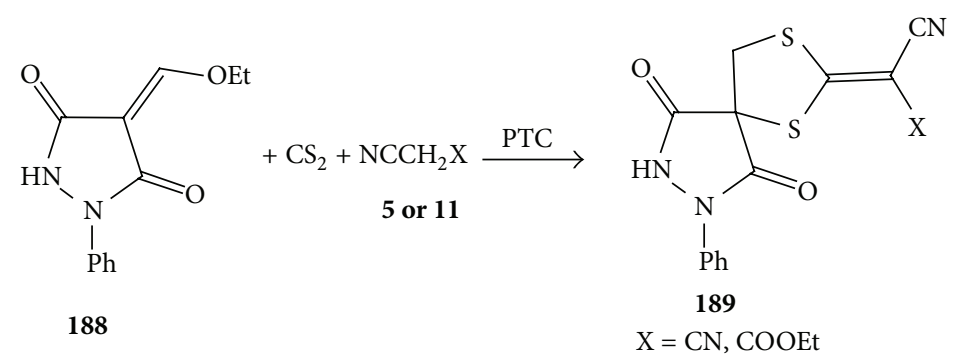

Figure 72
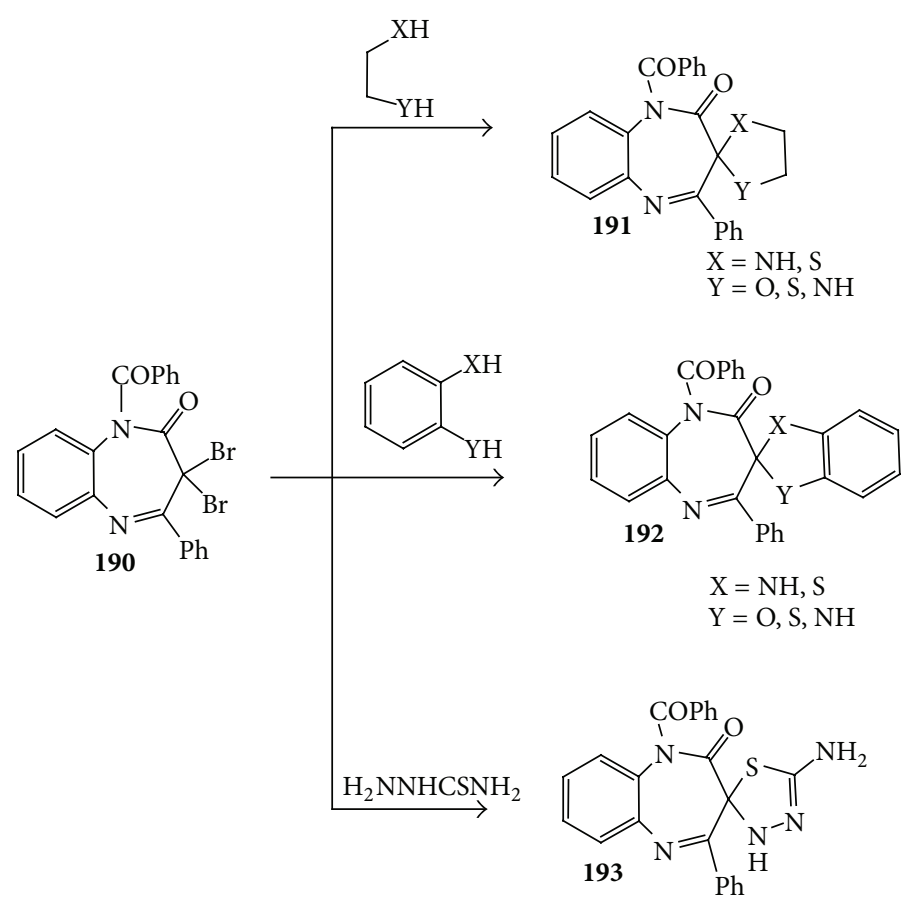

FIGURE 73<smiles>c1cc[nH]c1</smiles>

161<smiles>[R17]CCC</smiles><smiles>[R]n1cccc1</smiles>

162<smiles>[R]c1ccc[nH]1</smiles>

163<smiles>[R]c1cc[nH]c1</smiles>

164

FigURE 74<smiles>[R]OCC=CC[NH+]1C(=O)CCC1=O</smiles>

198

199<smiles>[R]OCC=CCN1C(=O)CCC1=O</smiles>

200

Figure 75 


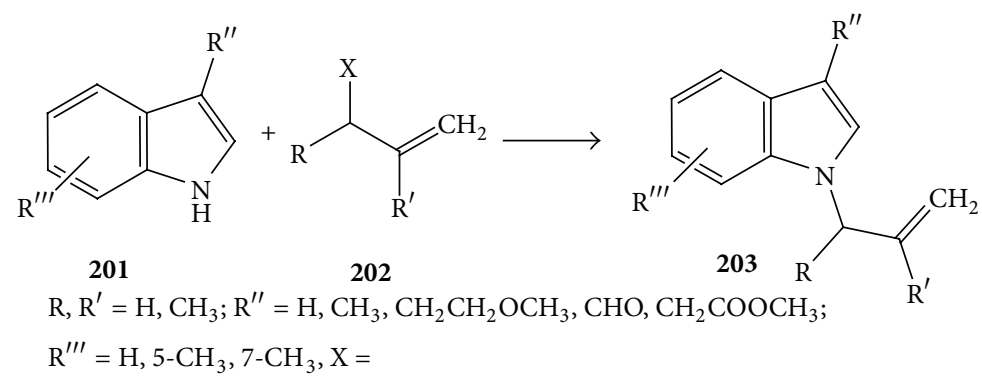

Figure 76

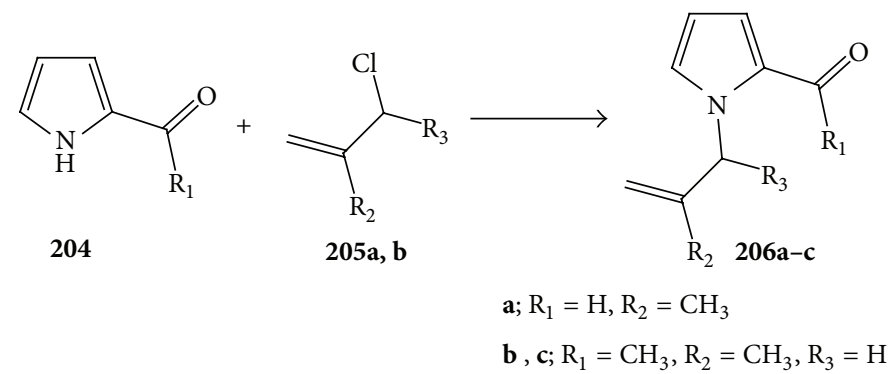

FIGURE 77<smiles>[R]C(=O)c1ccc[nH]1</smiles>

204

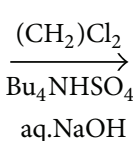<smiles>[R]C(=O)c1cccn1CCCl</smiles>

207

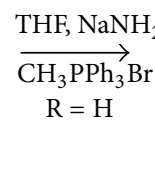

(a) $\mathrm{R}=\mathrm{H}$

(b) $\mathrm{R}=\mathrm{CH}_{3}$

FIGURE 78

The reaction was accomplished in diethyl ether via a phase transfer process in which 18-crown-6 was employed as the transfer agent and $t$-BuOK as the base [68] (Figure 76).

2-Substituted 1-allylpyrroles 206a-c were easily prepared from reaction of 2-formylpyrrole or 2-acetylpyrrole 204 with either 3-chlorobut-1-ene 205a or 3-chloro-2-methylprop1-ene 205b, respectively, under phase transfer conditions (toluene/NaOH/TBAHSO ${ }_{4}$ ) [69] (Figure 77).

Treatment of pyrroles 204 with 1,2-dichloroethane in presence of $50 \% \mathrm{NaOH}$ and $\mathrm{TBAB}$ as a catalyst yielded the corresponding N-chloroethyl-pyroles 207 in excellent yield which was transformed into 1,2-divinylpyrroles 208 via its reaction with sodamide using solid-liquid technique (THF/NaNH$H_{2} / \mathrm{CH}_{3} \mathrm{Ph}_{3} \mathrm{Br}$ ) [70] (Figure 78).

Synthesis of a series of N-alkylpyrrolidino[59]fullerenes was achieved via the combination of PTC without solvent and microwave irradiation technique. Thus, 2-phenylpyrrolidinofullerene 209 was treated with benzyl, $p$-nitrobenzyl, $p$-methyloxy-carbonylbenzyl, n-octyl, or allyl bromides in microwave oven in presence of $\mathrm{K}_{2} \mathrm{CO}_{3}$ and TBAB to yield the corresponding $\mathrm{N}$-alkylpyrrolidino[59]fullerenes 210 [71] (Figure 79).

1,3-Bis[2-(aryl)indol-1-yl]propanes 213 and 1,3-bis[3(aryl)-5-(aryl)pyrazol-1-yl]-propanes 214 were prepared 


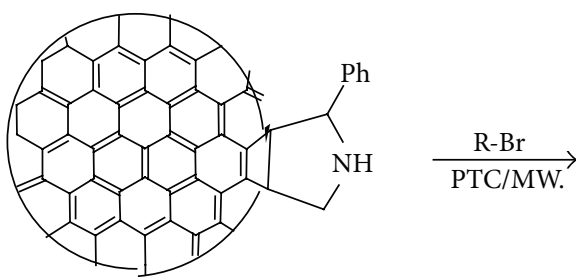

209

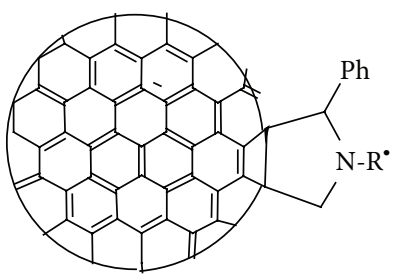

210

FIGURE 79<smiles>Brc1cc2ccccc2[nH]1</smiles>

211<smiles>[Al]c1cc2ccccc2n1CCCn1c([Al])cc2ccccc21</smiles>

213<smiles>[Al]C1=NN(CN2N=C([Al])CC2[Al])C([Al])C1</smiles>

214

Figure 80<smiles>N#CCc1c[nH]c2ccccc12</smiles>

215<smiles>N#CCc1c[nH]c2ccccc12</smiles>

216a 216b

Figure 81

from reaction of appropriate 2-arylindoles 211 and 4,5-dihydropyrazoles 212 , respectively, with 1,3-dibromopropane, via liquid-liquid technique using TBAHS as a catalyst, benzene as the organic phase, and $50 \% \mathrm{KOH}$ as an aq. phase [72] (Figure 80).

Indole-2-acetonitrile 215 was treated with $\left(\mathrm{CH}_{3}\right)_{2} \mathrm{CO}_{3}$ in a mixture of $\left[\mathrm{DMF} / \mathrm{TBAB} / \mathrm{K}_{2} \mathrm{CO}_{3}\right]$ to afford 1-methylindole2-acetonitrile 216a and 2-(1-methylindole-3-yl)propionitrile 216b [73] (Figure 81).

Alkylation of indole 217, 2,3-dimethylindole 218 with chlorodifluromethane under liquid-liquid technique $\left(\mathrm{CH}_{2} \mathrm{Cl}_{2} / \mathrm{aq}\right.$. NaOH/BzTEACl$)$ afforded the corresponding 1-alkylated derivatives 219 and 220, respectively [74] (Figure 82).

Barraja et al. heated 2-substituted-3-bromoindoles 221 with excess of different nucleophiles, solid $\mathrm{KOH}$, and dibenzo-18-crown as a phase transfer catalyst to afford the corresponding 3-substituted indoles $\mathbf{2 2 2}$ in a satisfactory increase of yield [75] (Figure 83).

Carbazole 223 was alkylated with 1,6-dibromohexane in 1:1 molar ratio to give $\mathrm{N}$-alkyl derivative $\mathbf{2 2 4}$ in a mixture of (benzene/NaOH/TBAB) [76] (Figure 84).

Alkylation of pyrazole 225 with cyclopentyl or cyclohexyl bromides without solvent with PTC system $(\mathrm{KOH} /$ 


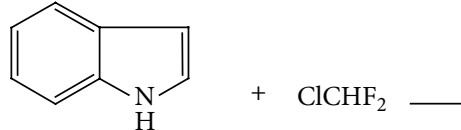

217<smiles>Cc1[nH]c2ccccc2c1C</smiles>

218<smiles>FC(F)n1ccc2ccccc21</smiles>

219<smiles>Cc1c(C)n(C(F)F)c2ccccc12</smiles>

220

FIGURE 82

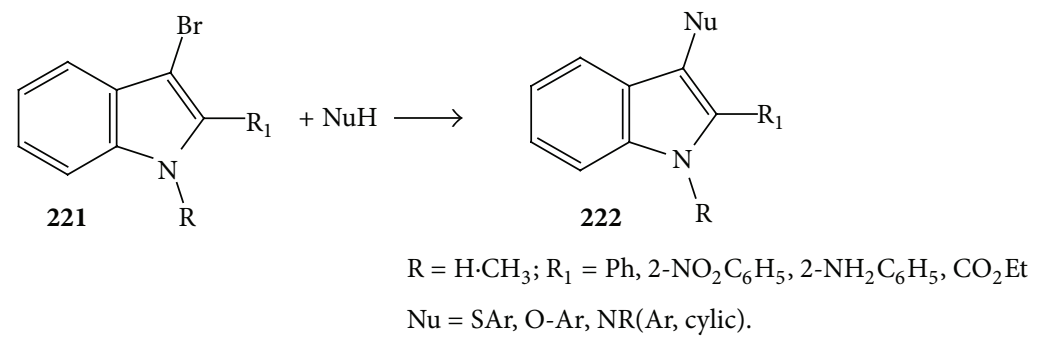

FIgURE 83<smiles>CC(CC(Br)(Br)CBr)c1ccc2c(c1)c1ccccc1n2CCBr</smiles>

FIGURE 84

TBAB) afforded the corresponding 1-cyclopentyl or 1-cyclohexylpyrazoles 226a, b, respectively. Likewise treatment of pyrazole with 1,2-dibromoethane in $1: 1$ or $2: 1$ molar ratio afforded 1-bromoethylpyrazole or 1,2 bispyrazolylethane 227, 228 [76](Figure 85). Also, alkylation reaction of $1 H$-pyrazole 225 with linear or branched alkyl halide in liquid-liquid PTC system gave 1-alkyl-1H-pyrazole 229 [77] (Figure 85).

The reaction of 3-tert-butylpyrazole 230 with dibromomethane afforded bis(tert-butylpyrazol-1-yl)methane $\left[\mathrm{CH}_{2}(3-\right.$ $\mathrm{t}-\mathrm{BuPz})_{2}$ ] 231. However, the reaction of 3-isopropylpyrazole 232 with dibromomethane under the same condition yielded three isomers, namely, bis(5-isopropylpyrazol-1-yl)methane $\left[\mathrm{CH}_{2}(5 \text {-isoPrPz })_{2}\right]$ 233, (3-isopropylpyrazol-1-yl-5' -isopropylpyrazol-1' - yl)methane $\left[\mathrm{CH}_{2}\left(3-\mathrm{iPrPz}-5^{\prime}\right.\right.$ isoPrPz $)_{2}$ ] 234, and bis(3-isopropylpyrazol-1-yl) methane $\left[\mathrm{CH}_{2}(3-\mathrm{iPrPz})_{2}\right] 235$ [78] (Figure 86).
N-Substituted-3,5-diarylpyrazolines 237 and 238 were prepared via liquid-liquid system in $\left(\mathrm{CHCl}_{3}\right.$ or benzene/aq. $\mathrm{KOH} / \mathrm{TBAB}$ ) using alkyl and allyl halides, respectively, as alkylating agents for pyrazoles 236 [79] (Figure 87).

$\mathrm{N}$-Alkylation reactions of 4,9-dihydro-9-methyl-4,10,10trioxo-1(2H)-pyrazolo[3,4-c][2,1]-benzothiazepine 239 with dimethyl sulphate, diethyl sulphate, benzyl bromide, benzyl chloride, phenacyl bromide, phenacyl chloride, or cyclohexyl bromide under the liquid-liquid PTC condition of (toluene/ $\mathrm{NaOH}(25 \%) / \mathrm{BTEACl}, \mathrm{TBAB}$, or $\mathrm{TBAHSO}_{4}$ ) produced 1- and 2-alkylated isomers $240 a, b$. The ratios between these two isomers were calculated [80] (Figure 88).

A number of 4-substituted pyrazolo[3,4-d]pyrimidines 241 have been reacted with (2-acetoxyethoxy)methyl bromide using liquid-liquid and solid-liquid techniques. The influences of the solvent and catalyst have been studied [81, 82] (Figure 89). 

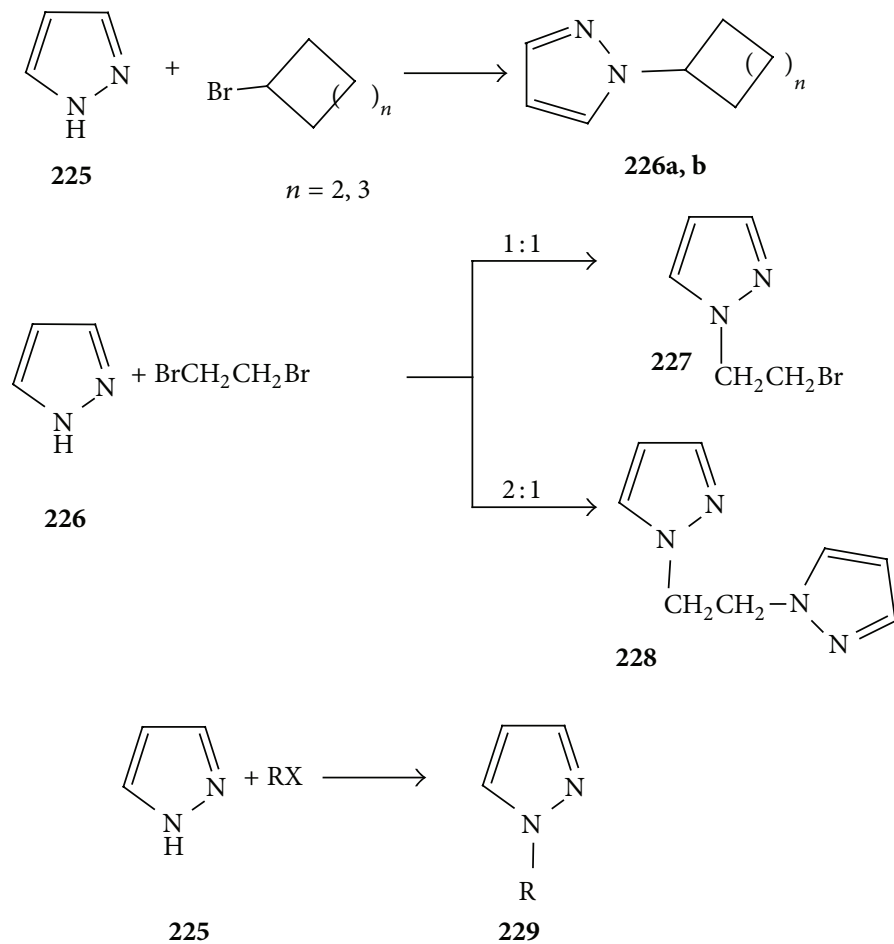

$\mathrm{R}=$ linear or branched alkyl $\mathrm{C}_{1}-\mathrm{C}_{6}$

FIGURE 85

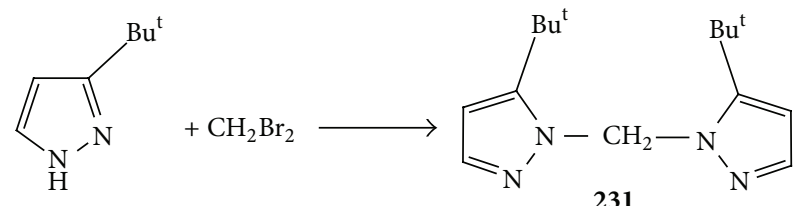

230
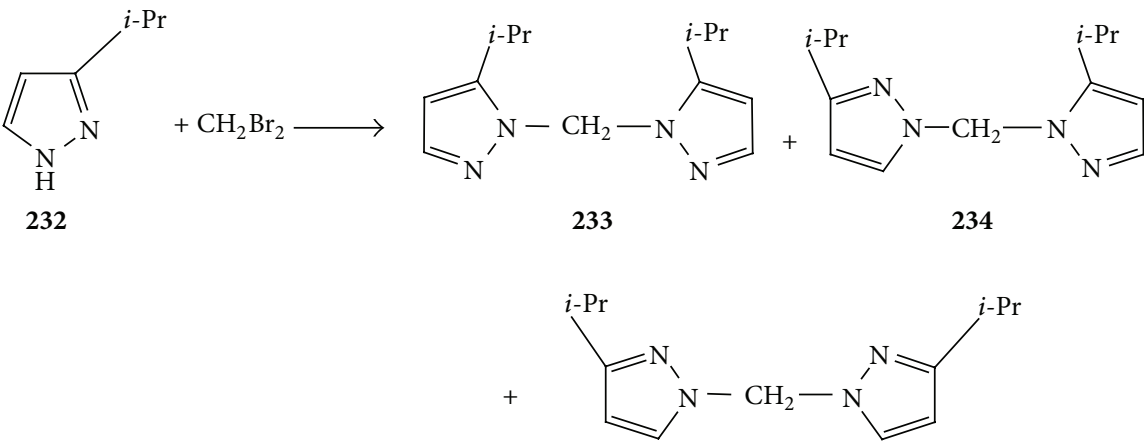

235

FIgURE 86

Imidazole $\mathbf{2 4 3}$ was alkylated with different alkyl halides under solid-liquid PTC in absence of solvent where the $\mathrm{N}$-alkyl imidazoles 244a and imidazolium salts 244b were obtained [83] (Figure 90).

1-Alkyl $\left(\mathrm{C}_{4}-\mathrm{C}_{14}\right)$ imidazoles 245 were obtained from the alkylation of imidazole 243 with the appropriate n-bromoalkane via PTC technique (benzene/KOH/TBAI) [84] (Figure 91).
1-Alkyl-2-methyl-2-imidazolines 247 were obtained in good to excellent yields by alkylation of 2-methyl-2-imidazoline $\mathbf{2 4 6}$ with organic halides in the absence of solvent [85] (Figure 92).

Using the PTC condition such as $\left(\mathrm{CH}_{2} \mathrm{Cl}_{2} / \mathrm{KOH} / \mathrm{TEBA}\right)$, a mixture (1:1) of $\mathrm{N}$-alkylated 5-nitroimidazoles $250 \mathbf{a}$, $\mathbf{b}$ or 6-nitrobenzimidazoles 251a, $\mathbf{b}$ was obtained by the reaction of 5-nitroimidazoles $\mathbf{2 4 8}$ or 6-nitrobenzimidazoles $\mathbf{2 4 9}$ with 


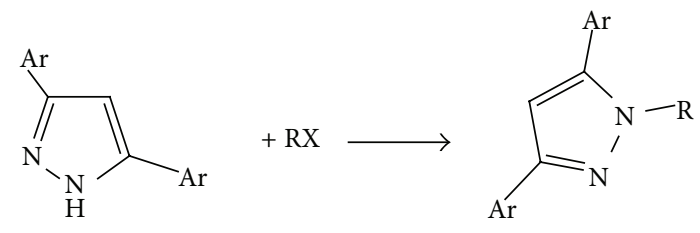

236

237<smiles>[Y]C(C)C=C[CH+]</smiles>

236<smiles>C=CCn1nc([Al])cc1Br</smiles>

238

Figure 87<smiles>CN1c2ccccc2C(=O)c2c[nH]nc2S1(=O)=O</smiles>

239<smiles>[R]O[R16](=O)(=O)OCC</smiles><smiles>[R]N1c2ccccc2C(=O)c2cnn([R19])c2S(=O)(=O)N1[R]</smiles>

240a<smiles>[R]N1c2ccccc2C(=O)c2cn([R])nc2S1(=O)=O</smiles>

240b

FIGURE 88<smiles></smiles><smiles>[R]c1ncnc2nn(COC(C)=O)cc12</smiles>

FigURE 89

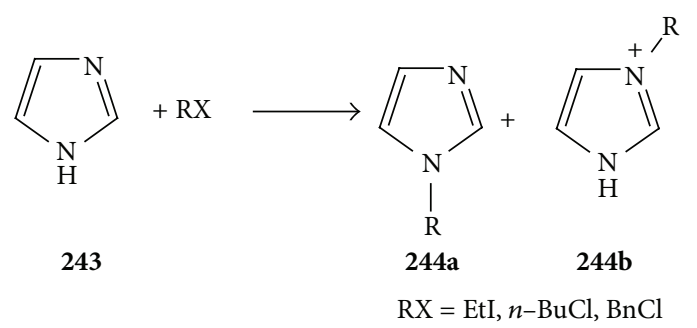

FIgURE 90 


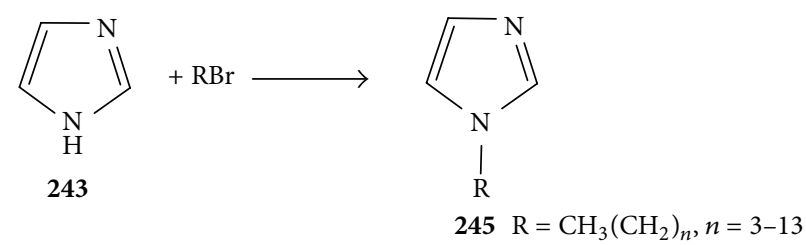

FIGURE 91<smiles>[R2][C@H]1C[C@H](C)N(C)C=CN1[R]</smiles>

$246 \mathrm{H}$

247

FIgURE 92<smiles>Cn1cnc([N+](=O)[O-])c1</smiles>

248

250a

250b<smiles></smiles>

Figure 93<smiles>[R]c1c[nH]c([R])n1</smiles>

252a, b

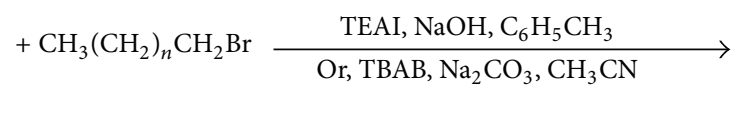

253<smiles>[R]c1cn(CCCCCCCCCC)c([R])n1</smiles>

254

a, $\mathrm{R}_{1}=\mathrm{CH}_{3}, \mathrm{R}_{2}=\mathrm{H}$

b, $\mathrm{R}_{1}=\mathrm{CH}_{3}, \mathrm{R}_{2}=\mathrm{NO}_{2}$

FIGURE 94<smiles>[R][PH2+]C(C)=O</smiles>

256<smiles>[R]n1c(C(C)=O)nc2ccccc21</smiles>

257

FIGURE 95 
<smiles>FC(F)[PH2+]c1nc2ccccc2[nH]1</smiles>

258<smiles>CC(C)C1C(c2ccccc2)=Nc2ccccc21</smiles>

259

Figure 96<smiles>[R]c1ncc[nH]1</smiles>

260 $+\mathrm{CH}_{3}\left(\mathrm{CH}_{2}\right)_{n} \mathrm{Br}$

261

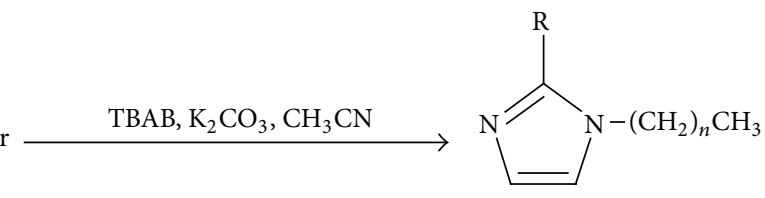

262

FIgURE 97<smiles>CC1(C)CC(=O)NO1</smiles>

263<smiles>CC1(C)CC(=O)N(Cc2ccccc2)O1</smiles>

264a

264b

Figure 98

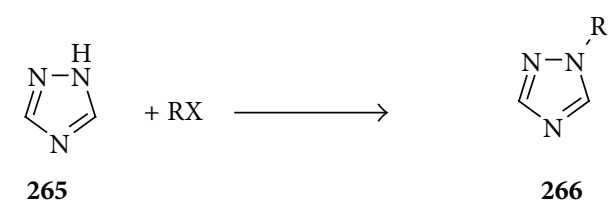<smiles>[R17]CI</smiles>

267<smiles>c1ccc2[nH]nnc2c1</smiles>

+ ArX<smiles>CC</smiles><smiles>[R]n1nnc2ccccc21</smiles>

268<smiles>[Al+]n1nc2ccccc2n1</smiles>

269a
$269 b$

FIGURE 99 


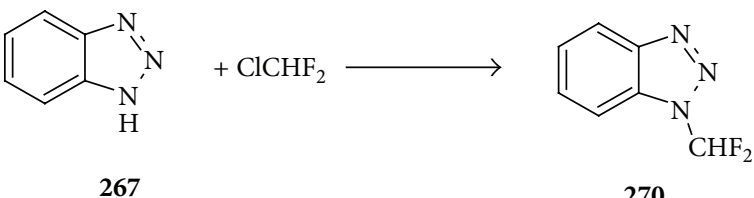

FIGURE 100

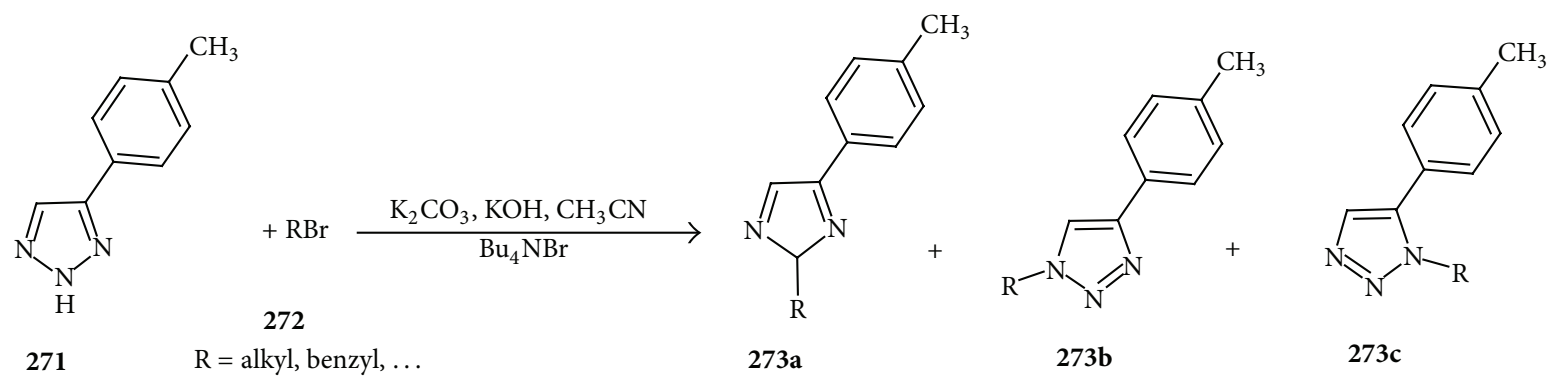

FIGURE 101

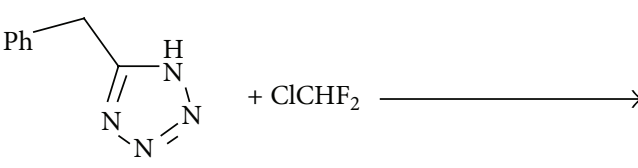

274<smiles>CCn1nnc(Cc2ccccc2)n1</smiles>

$275 a$ 275b

FIGURE 102

methyl iodide or benzyl chloride, respectively, in a satisfactory yield [86] (Figure 93).

Different imidazole compounds such as 2-methylimidazole 252a and 2-methyl-4-nitro imidazole 252b were reacted with different alkyl bromides 253 in an alkaline media, at reflux temperature and in the presence of tetraethylammonium iodide (TEAI) or TBAB as PTC and afforded 1alkyl imidazole derivatives $\mathbf{2 5 4}$ which exhibited a variety of valuable pharmacological properties [87] (Figure 94).

N-Alkyl-2-acetylbenzimidazoles 257 have been obtained in over $90 \%$ yield by alkylating 2-acetylbenzimidazole 256 using the PTC mixture of $\left(\mathrm{CH}_{3} \mathrm{CN} / \mathrm{K}_{2} \mathrm{CO}_{3} / \mathrm{TEBACl}\right)$ at room temperature [88] (Figure 95).

2-Phenylbenzimidazole 258 was alkylated with chlorodifluromethane under liquid-liquid condition $\left(\mathrm{CH}_{2} \mathrm{Cl}_{2} / \mathrm{aq}\right.$. $\mathrm{NaOH} / \mathrm{BzTEACl}$ ) to give 1-difluoromethyl-2-phenylbenzimidazole 259 [75] (Figure 96).

The $\mathrm{N}$-alkylated imidazole derivatives $\mathbf{2 6 2}$ were achieved by treating compounds $\mathbf{2 6 0}$ with different alkyl bromide
261 under PTC condition $\left(\mathrm{TBAB} / \mathrm{K}_{2} \mathrm{CO}_{3} / \mathrm{CH}_{3} \mathrm{CN}\right)$ [89] (Figure 97).

Competitive $\mathrm{N}$ versus $\mathrm{O}$ benzylation of 5,5-dimethyl3-isoxazolidinone 263 using $\left(\mathrm{CH}_{2} \mathrm{Cl}_{2} / \mathrm{NaOH}\right)$ using different PTC catalysts was studied. The ratio of N-/Oalkylations $264 \mathbf{a} / 264 \mathbf{b}$ was $2 / 3$ for most cases of catalysts [90] (Figure 98).

Alkylation of 1,2,4-triazole 265 and benzotriazole 267 has been performed either in basic media under solvent free PTC conditions or in absence of base by conventional and microwave heating. Several parameters affecting the selectivity have been studied [91]. Arylation of $1 H-1,2,3-$ benzotriazole 267 with activated aryl halides in a medium of aromatic hydrocarbons under PTC condition using inorganic bases and acetyltrimethylammonium bromide as a phase transfer catalyst was studied. Both N(1)- and N(2)-arylated products $269 \mathrm{a}$ and $269 \mathrm{~b}$, respectively, have been isolated. Their ratio has depended on the nature of the employed base and the reactivity of arylating agent [92] (Figure 99). 
<smiles>[R]Sc1nnnn1[Al-]</smiles><smiles>O=[N+]([O-])c1ccc(-n2cncn2)cc1</smiles>

Pyrazole, imidazole, 1, 2, 4-triazole, indazole, benzotriazole

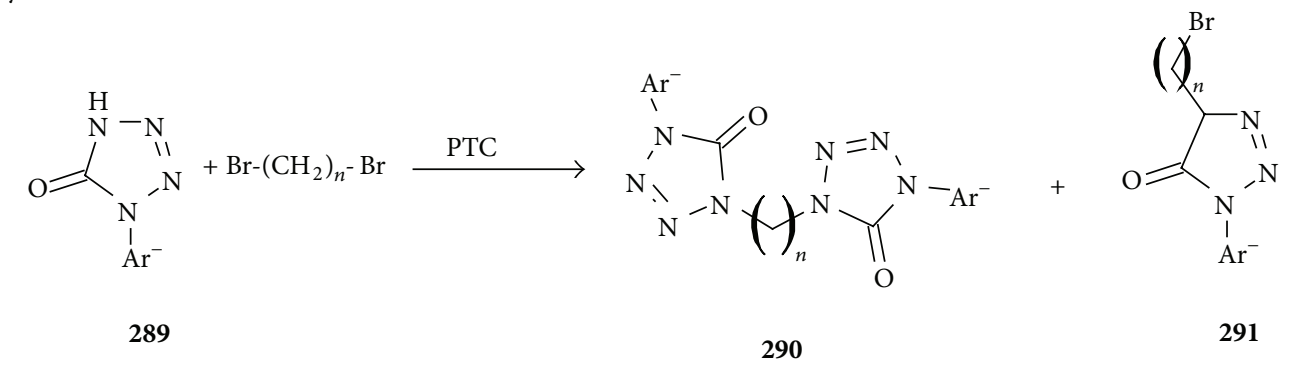

FIgURE 103<smiles>CCO[C@H]1OC(n2cnc3c(NC(C)=O)ncnc32)C(OC(C)C)C1O[Na]</smiles>

292

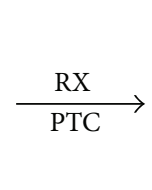

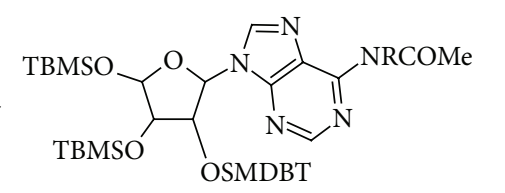

293a

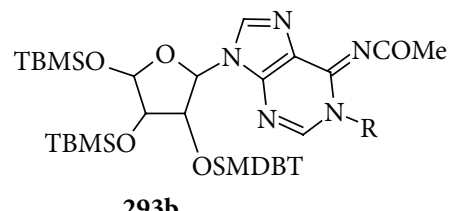

293b

$$
\mathrm{R}=\mathrm{Me}, \mathrm{PhCH}_{2}, \mathrm{CH}_{2}-\mathrm{CH}=\mathrm{CH}_{2}, \mathrm{X}=\mathrm{Br}, \mathrm{I}
$$

FIgURE 104

Treatment of benzotriazole $\mathbf{2 6 7}$ with chlorodifluromethane under liquid-liquid conditions $\left(\mathrm{CH}_{2} \mathrm{Cl}_{2}\right.$ /aq. $\mathrm{NaOH} /$ BzTEACl) has afforded 1-(difluoromethyl)- $1 H$-benzotriazole 270 [74] (Figure 100).

Reaction of 4-substituted-1,2,3-triazol 271 with alkyl bromide 272 under basic condition using PTC $\mathrm{Bu}_{4} \mathrm{NBr}$ produced $\mathrm{N}$-substituted 1,2,3-triazole derivatives 273a-c [93] (Figure 101).

The alkylation of 5-benzyl-1H-tetrazole 274 under liquidliquid technique such as $\left(\mathrm{CH}_{2} \mathrm{Cl}_{2} / \mathrm{aq}\right.$. $\left.\mathrm{NaOH} / \mathrm{BzTEACl}\right)$ gave a mixture of 5-benzyl-1-difluoromethyl-1,2,3,5-tetrazole 275a in 23\% and 4-benzyl-1-difluoromethyl-1,2,3,5-tetrazole $\mathbf{2 7 5 b}$ in 15\% [74] (Figure 102).

A series of 5-alkylthio-1-aryltetrazoles 277 were prepared by alkylation reaction of the corresponding 1-aryltetrazole5-thiols 276 with alkyl bromides under solid-liquid PTC technique [94], whereas without solvent and also under PTC technique several N-p-nitrophenylazoles 279-288 were synthesized by direct arylation of the corresponding azolo compound $\mathbf{2 7 8}$ with $p$-fluoronitrobenzene [95], while the alkylation reaction of 1-aryltetrazol-5-ones 289 with dibromoalkanes under both liquid-liquid and solid-liquid PTC 


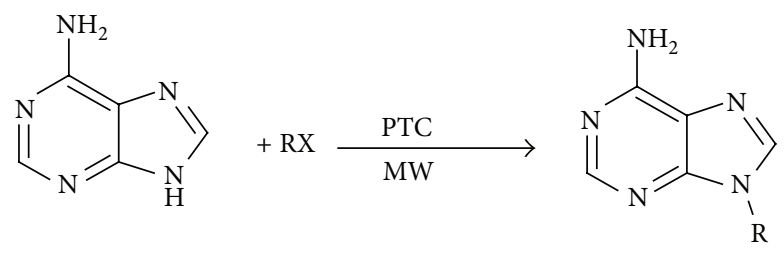

294

295

FIgURe 105<smiles>COCc1nnc(N)s1</smiles>

FIGURE 106<smiles>COc1ccc(/C=C2\NC(=S)C(c3ccccc3)C2=O)cc1OC</smiles>

299

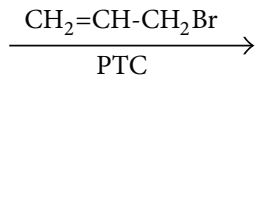

FIGURE 107<smiles>[R]c1cc([Y])[nH]n1</smiles>

301-303<smiles>C=CCN1C(=S)C(c2ccccc2)C(=O)/C1=C\c1ccc(OC)c(OC)c1</smiles>

300<smiles>[R]c1cc([R])n(CCN)n1</smiles>

304-306

$301,304 \mathrm{R}=\mathrm{R}^{\prime}=\mathrm{H} ; 302,305 \mathrm{R}=\mathrm{CH}_{3}, \mathrm{R}^{\prime}=\mathrm{H} ;(\mathrm{a}), \mathrm{R}^{\prime}=\mathrm{CH}_{3}, \mathrm{R}=\mathrm{H}$; (b) 303, 306 R= $\mathrm{R}^{\prime}=\mathrm{CH}_{3}$

FIGURE 108<smiles>[Y]c1cc([R])[nH]n1</smiles>

301-303<smiles>[R]c1cc([Y])n(CCCl)n1</smiles>

$\mathrm{H}_{2} \mathrm{CH}_{2} \mathrm{Cl}$<smiles>[Y]c1cc([R])n(C=C)n1</smiles>

307-309

$301,304,307 \mathrm{R}=\mathrm{R}_{1}=\mathrm{H} ; 302,305,308 \mathrm{R}=\mathrm{H}, \mathrm{R}_{1}=\mathrm{Me}, \mathrm{R}=\mathrm{Me}, \mathrm{R}_{1}=\mathrm{H} ; 303,306,309 . \mathrm{R}, \mathrm{R}_{1}=\mathrm{Me}$

Figure 109 


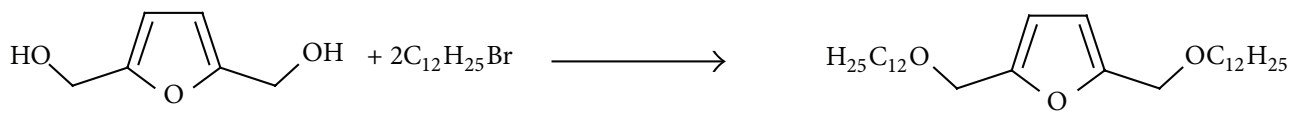

310

311

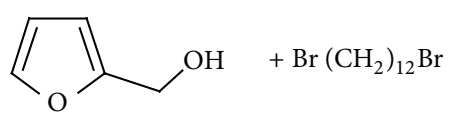<smiles>COC(C)OCc1ccco1</smiles>

312

313

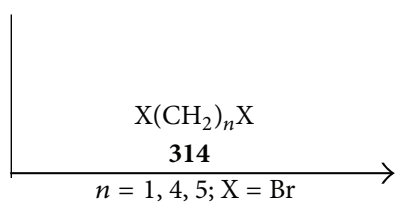<smiles>C=C(CC)COC(C)OCc1ccco1</smiles>

315

FIGURE 110<smiles>COC1OC(OC)C(O)C1O</smiles>

316

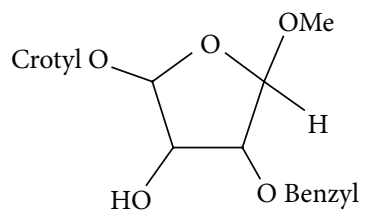

317<smiles>COc1ccc(/C=C2\NC(=S)N(c3ccccc3)C2=O)cc1OC</smiles>

318
FIgURe 111

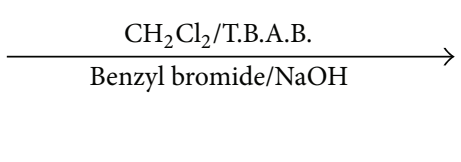

FIgURE 112 $\mathrm{BrCH}_{2} \mathrm{CH}_{2} \mathrm{Br}$

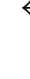<smiles>C=[Al]</smiles>

322

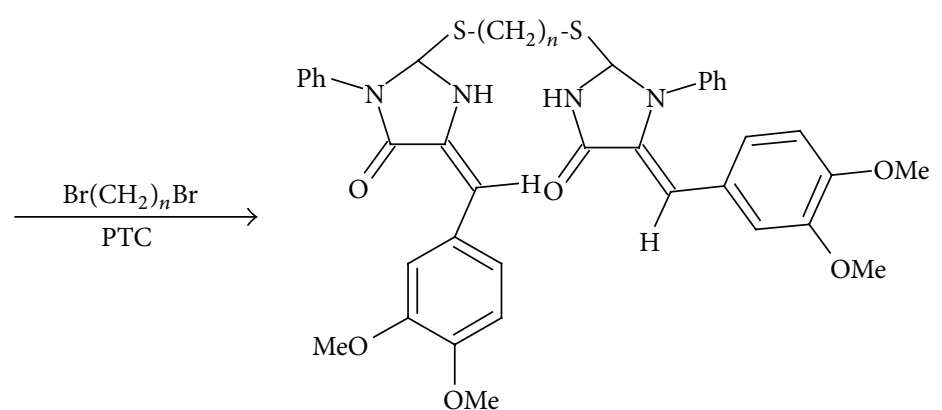

$319 a, b$<smiles></smiles>

320

$$
\begin{aligned}
\mathrm{R}= & \mathrm{Me}, \mathrm{Et}, \mathrm{CH}_{2} \mathrm{CHO}, \mathrm{CH}_{2} \mathrm{CO}_{2} \mathrm{Et}, \mathrm{CH}\left(\mathrm{CO}_{2} \mathrm{Et}\right)_{2}, \\
& \mathrm{COMe}, \mathrm{CO}_{2} \mathrm{Et}, p-\mathrm{NO}_{2}-\mathrm{C}_{6} \mathrm{H}_{4}-\mathrm{N}=\mathrm{N}
\end{aligned}
$$

FIGURE 113 


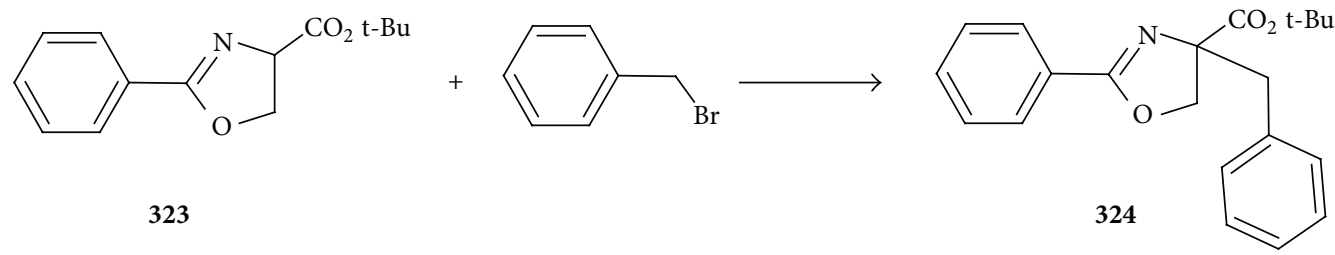

FigURE 114<smiles>[R]c1cc([R])c2c(=O)n([Y])c(=S)[nH]c2n1</smiles>

325<smiles>O=C(OCC1OC(Br)C(COC(=O)c2ccccc2)C1OC(=O)c1ccccc1)c1ccccc1</smiles>

326

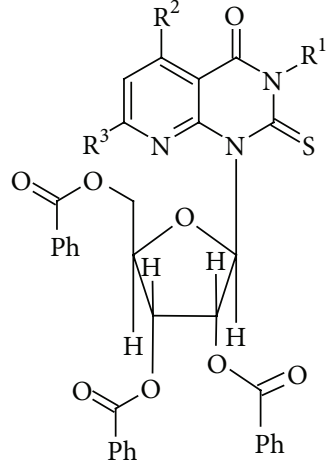

327

Figure 115

systems provided a convenient route to prepare bis-tetrazolon derivatives 290 and 4-bromoalkyl derivatives 291 [96] (Figure 103).

N-Acetylated adenosine 292 was alkylated with benzyl bromide, methyl iodide, or allyl bromide in the presence of tetrabutylammonium bromide as a PTC catalyst in $\mathrm{CH}_{2} \mathrm{Cl}_{2} / \mathrm{NaOH}$ to give the corresponding $\mathrm{N}$-alkyl-6-N-acetyl adenosines 293a, b [97] (Figure 104).

$\mathrm{N}$-alkylation reaction of adenine 294 took place with different alkyl halides under the phase transfer conditions using microwave irradiation assistance [98] (Figure 105).

5-Aryloxymethyl-2-[(2-chlorophenyl)oxyacetylamido]1,3,4-thiadiazoles $\mathbf{2 8 0}$ are synthesized from reaction of 2-chlorophenyloxyacetyl chloride 297 with 5-aryloxymethyl2-amino-1,3,4-thiadiazoles 296 under liquid-liquid condition using PEG-400 as a catalyst [99] (Figure 106).

$\mathrm{N}$-Alkylation of 3-phenyl-2-thiohydantoin-5-arylidene derivative 299 has been carried out by using monohalocompounds such as allyl bromide as an alkylating agent. The reaction was proceeded through nucleophilic displacement under PTC conditions of solid-liquid phases such as a mixture of anhydrous potassium carbonate, dioxane, and TBAB as a heterogeneous catalyst. The corresponding alkylated product 300 was obtained in a good yield [42] (Figure 107).

Alkylation reaction of pyrazoles 301-303 with 2chloroethylamine under the condition of phase-transfer catalysis was carried out in a liquid-solid system using benzene as an organic solvent and BTEAC as a catalyst. Reaction between equimolar amounts of reactants led to poor yields (20-40\%) of the alkylated products 304-306 due to concurrent dehydrochlorination of 2-chloroethylamine. The yield was considerably decreased in going from pyrazole (301) to 3,5 dimethylpyrazole (303) whereas the maximal yield of alkylated products $(70-80 \%)$ was obtained by using one of the following ratios: pyrazole: $\mathrm{NaOH}: 2$ chloroethylamine $1: 2: 2,3$-(5)methylpyrazole: $\mathrm{NaOH}: 2$ chloroethylamine $1: 3: 3$, or 3,5-dimethylpyrazole : $\mathrm{NaOH}: 2$ chloroethylamine 1:5:5 [100] (Figure 108).

In the $\mathrm{N}$-alkylation reaction of pyrazole, 3-(5)-methylpyrazole, and 3,5-dimethylpyrazole 301-303 with dichloroethane (DCE), the dehydrochlorination of the obtained 1( $\beta$-chloroethyl)pyrazoles has been carried out to give the corresponding products $\mathrm{N}$-vinylpyrazoles 307-309 (Figure 9) in low yield. Attempts to carry out the reaction under standard conditions (water/benzene/ $\mathrm{NaOH} / \mathrm{TEBAC}$ ) did not lead to the desired result. The yield of all products was sharply increased when benzene was replaced with an excess of dichloroethane. The reaction investigation showed that the ease of alkylation depends strongly on the basicity of the pyrazole. The introduction of an electron-donating substituent (e.g., Me) into the molecule of pyrazole 301 increases the electron density at the "pyrazole" nitrogen atoms. As a result, deprotonation was hindered and the base was consumed in elimination of dichloroethane. It must also be mentioned that a 5- to 7-folds excess of dichloroethane was necessary to obtain optimal yields on alkylation of compounds 301-303 [101] (Figure 109).

5.1.2. O-Alkylation or Acylation. 2,5-Didodecyloxymethylfuran 311 was obtained via alkylation of 2,5-dihydroxym- 


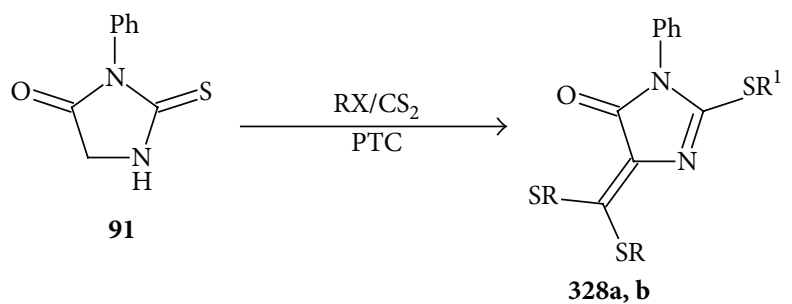

(a) $\mathrm{R}^{1}=\mathrm{H}, \mathrm{R}=\mathrm{Et}$

(b) $\mathrm{R}^{1}=\mathrm{R}=\mathrm{PhCH}_{2}$

FIGURE 116

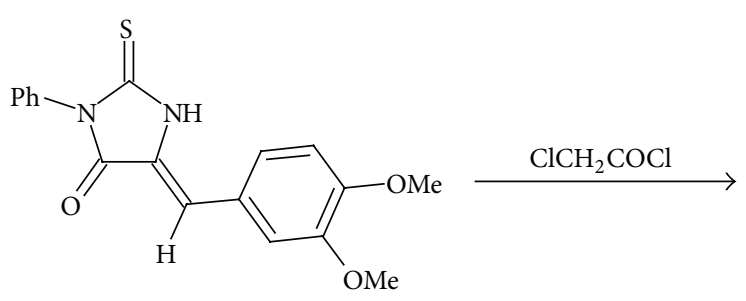

318<smiles>COc1ccc(/C=C2/C(=O)N(c3ccccc3)C(=S)N2C(=O)Cl)cc1OC</smiles>

329

FIgURE 117

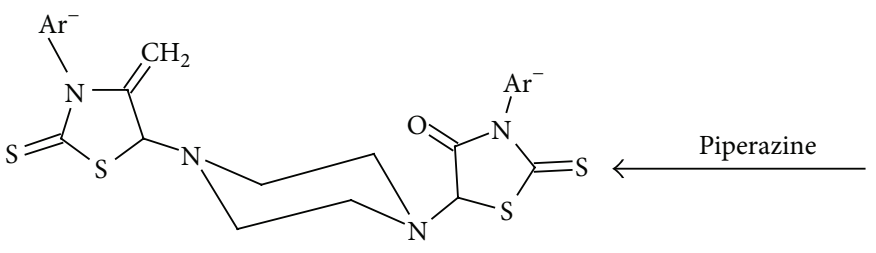

332

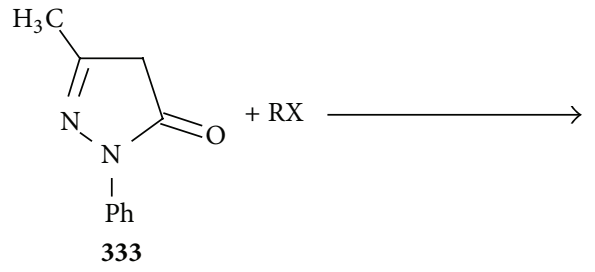

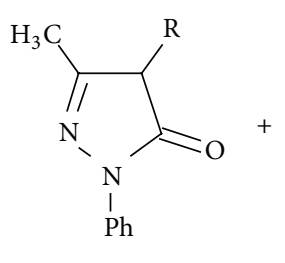

$334 a-e$

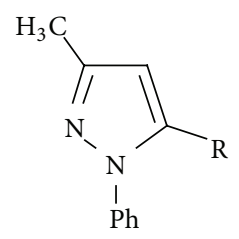

335a-e

FIGURE 119<smiles>BrCC(Br)(Br)CCc1nnnn1C1CCCCC1</smiles>

336<smiles>O=c1ccc2cc(O)ccc2[nH]1</smiles><smiles></smiles>

337

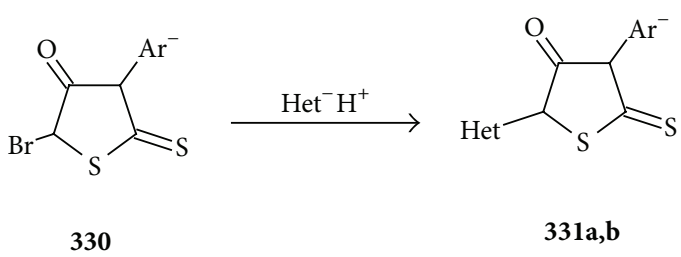

; b:<smiles>Nc1nc2ccccc2s1</smiles>

FIGURE 118 

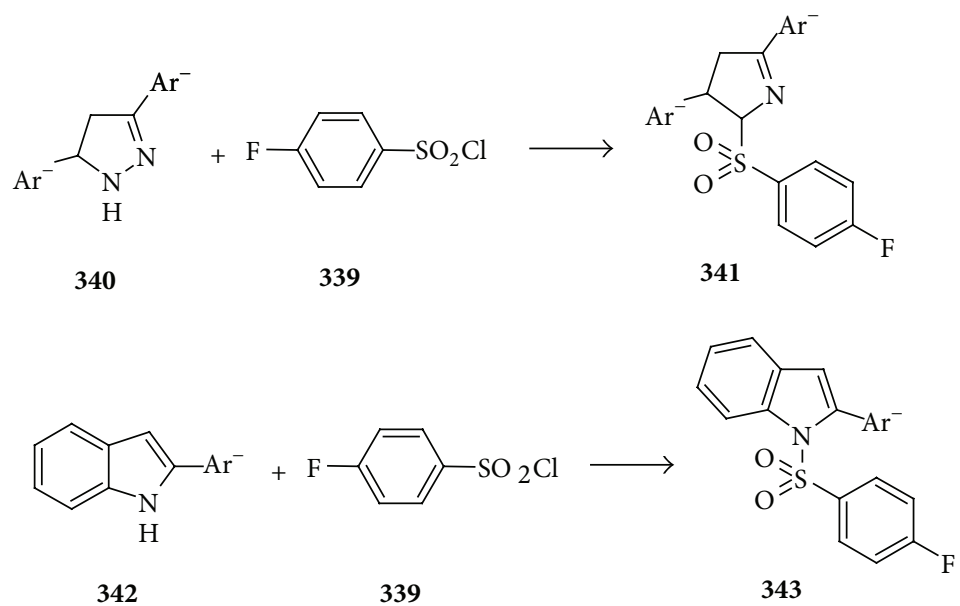

Figure 121<smiles>FC1(F)Oc2cc3c(cc2O1)CCC3</smiles>

344<smiles>Cc1noc(C)c1S(=O)(=O)Cl</smiles>

345<smiles>C1=CC2CCC=C1C2</smiles><smiles>Cc1noc(C)c1S(=O)(=O)N1c2cc3c(cc2NC1Cl)OC(F)(F)O3</smiles>

346

FIgURe 122

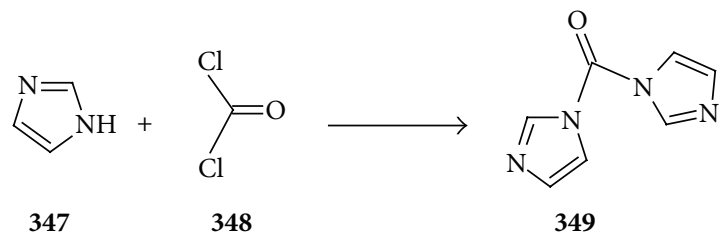

Figure 123

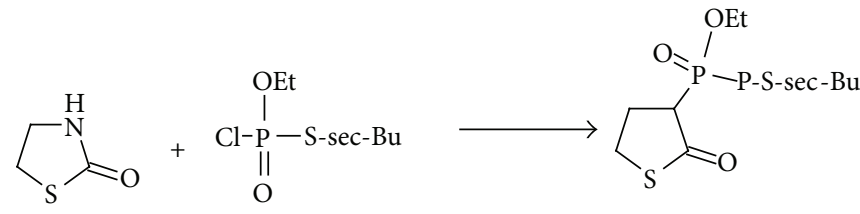

350

351

352

FIgURE 124

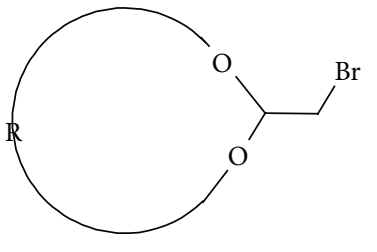

353

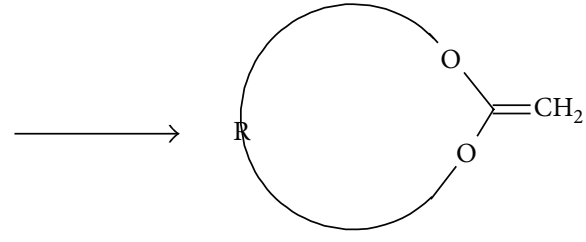

354

FIGURE 125 
<smiles>O=[N+]([O-])C1C(CCl)N=C2C=CC(Cl)=NN21</smiles>

355<smiles>CC(C)CC1N=C2C=CC(Cl)=NN2C1[N+](=O)[O-]</smiles>

357

FIgURE 126

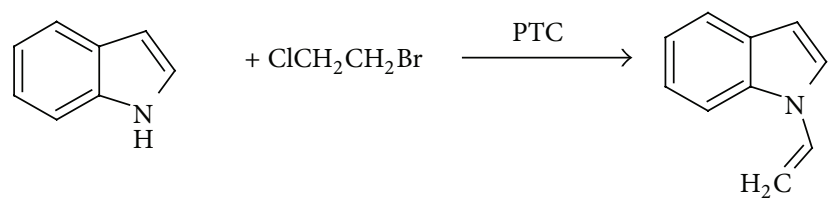

358

359<smiles>C=CSc1cn(C=C)c2ccccc12</smiles>

360<smiles>Sc1nc2ccccc2[nH]1</smiles>

361
362<smiles>C=CSc1nc2ccccc2n1C=C</smiles>

363

FIGURE 127

ethylfuran 310 with dodecyl bromide without solvent using (KOH/aliquat) as a catalyst, while alkylation of furfuryl alcohol 312 with 1,12-dibromododecane under the same PTC conditions produced the corresponding furanic diether 313 [102] (Figure 110).

Similarly, 2-furfuryl alcohol 312 was subjected to react with alkyl dihalides 314 using (benzene/KOH/polyethylene glycol (PEG-400)) to yield the corresponding difurfuryl diethers 315 [102] (Figure 110).

4-(benzyloxy)-2-[(2E)-but-2-en-1-yloxy]-5-methoxytetrahydrofuran-3-ol 317 was obtained from benzylation of 2-O-methyl-5-O-crotyl- $\alpha, \beta$-D-ribofuranoside 316 using benzyl bromide in aq. solution of $\mathrm{NaOH} / \mathrm{CH}_{2} \mathrm{Cl}_{2}$ and TBAB as a catalyst [103] (Figure 111).

5.1.3. S-Alkylation. PTC alkylation of arylidene derivative 318 using dihalocompounds such as ethylene dibromide or 1,3-dibromopropane afforded the thioalkylated dimers 319a, b, respectively, via s-alkylation pathway followed by dimerization reaction [42] (Figure 112).

5.1.4. C-Alkylation. Reaction of 2-thiono-N( $m$-tolyl)thiazolidin-4-one 320 with methyl bromide, ethyl bromide, chloroacetaldehyde, ethyl chloroacetate, diethyl malonate, acetyl chloride, ethyl chloroformate, or $p$-nitrobenzenediazonium tetrafluoroborate under solid-liquid system and using the heterogeneous mixture of (dioxan/ $\left.\mathrm{K}_{2} \mathrm{CO}_{3} / \mathrm{TBAB}\right)$ as a PTC yielded the corresponding 5-substituted thiazolidin-4-ones 321a-h and yielded the corresponding spiro thiazolidin-4one derivatives 322 when reacted with 1,2-dibromoethane under the same PTC condition [104] (Figure 113).

4-Benzyl-2-phenyl-2-oxazoline-4-carboxylic acid tertbutyl ester 324 was prepared via the alkylation of 2-phenyl-2oxazoline-4-carboxylic acid tert-butyl ester 323 with benzyl 
<smiles>O=C1CSC(=S)N1[Al+2]</smiles>

384

$\mathrm{Ar}=\mathrm{Ph}, p-\mathrm{MeO}-\mathrm{C}_{6} \mathrm{H}_{4}$

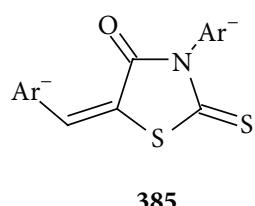

385

2-Thienyl- ; 2-turyl-

FIGURE 128<smiles>O=C1CSC(=S)N1[AlH2+]</smiles>

386
$\mathrm{HCHO}$ PTC

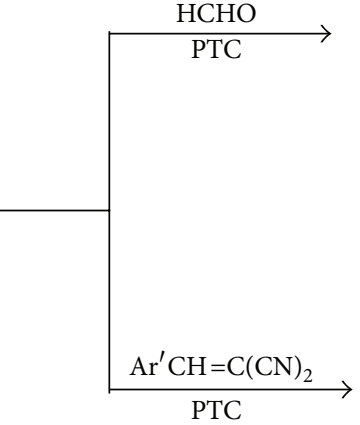<smiles>O=C1C(CO)SC(=S)N1[Al+]</smiles><smiles>N#CC(C#N)C([AlH2])C1SC(=S)N([Al-])C1=O</smiles>

388

$\mathrm{Ar}^{\prime}=\mathrm{Ph}, 4-\mathrm{N}(\mathrm{Me})_{2}-\mathrm{C}_{6} \mathrm{H}_{4}-$

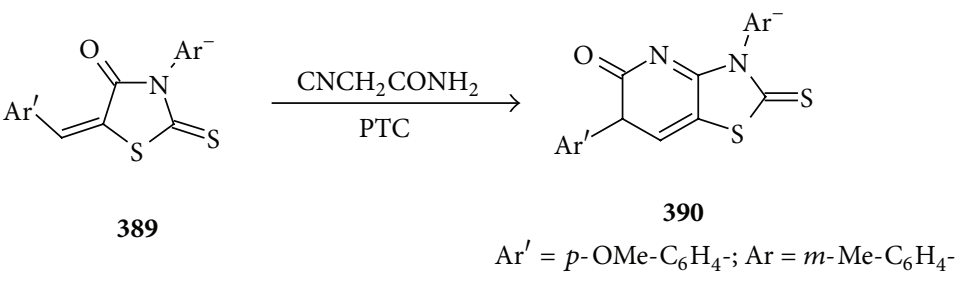

FIGURE 129<smiles>[R]C1CCC(=O)C1c1ccccc1</smiles><smiles>CC(Cl)NO</smiles>

391<smiles>C=CC</smiles><smiles>[R]C12C(c3ccccc3)C(=O)CC1C2(Cl)CC</smiles><smiles>[R]C1C(=O)C=CC(=O)N1c1ccccc1</smiles>

392<smiles>C[Te]</smiles><smiles>[R]c1c(Cl)ccc(=O)n1-c1ccccc1</smiles>

FIGURE 130 


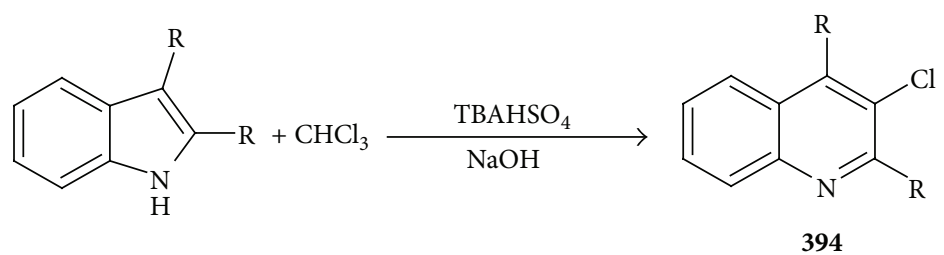

393<smiles>[R]c1[nH]c2ccccc2c1C=O</smiles><smiles></smiles><smiles>[R]c1nc2ccccc2c(C2OC2(Cl)Cl)c1Cl</smiles>

Figure 131

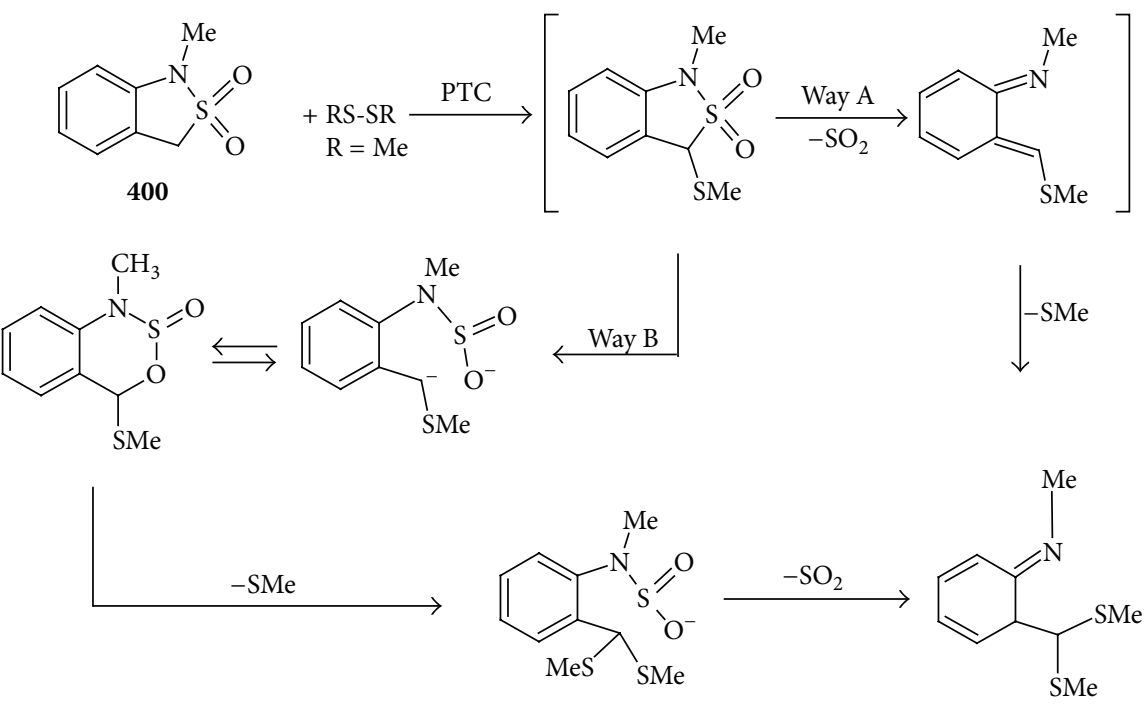

401

FIGURE 132

bromide using (toluene/ $\mathrm{KOH}(\mathrm{s}) / \mathrm{Cinchona}$ alkaloids) as a PTC [105] (Figure 114).

2-Thioxo-3,5,7-trisubstituted-1-(2,3,5-tri-O-benzoyl- $\beta$-Dribofuranosyl)pyrido[2,3-d]-pyrimidin-4(1H)-ones 327 have been prepared via phase transfer ribosylation of 2-thioxo3,5,7-trisubstituted pyrido[2,3-d]pyrimidin-4(1H)-ones 325 with 2,3,5-tri-O-benzoyl- $\beta$-D-ribofuranosyl bromide 326 in biphase solvents such as $\mathrm{CH}_{2} \mathrm{Cl}_{2} / 50 \%$ aq. $\mathrm{NaOH}$ using TBAB as a catalyst [106] (Figure 115).

In the presence of dioxane as a solvent under PTC conditions, one-pot reaction of 3-phenyl-2-thiohydantoin 91 with $\mathrm{CS}_{2}$ and halocompounds such as ethyl bromide or benzyl chloride afforded 5-[bis(ethylsulfanyl)methylidene]2-(ethylsulfanyl)-3-phenyl-3,5-dihydro- $4 \mathrm{H}$-imidazol-4-one 328a and 5-[bis(benzylsulfanyl)methylidene]-2-(benzylsulfanyl)-3-phenyl-3,5-dihydro- $4 H$-imidazol-4-one 328b, respectively [42] (Figure 116).

Acylation of imidazolones 318 using chloroacetyl chloride under PTC conditions yielded the corresponding acylated arylidene derivative 329 [42] (Figure 117).

5.1.5. Miscellaneous Alkylation. 5-Bromorhodanine derivative 330 was effectively used as an alkylating agent for some mono- or dianionic moieties containing $\mathrm{S}, \mathrm{N}$, or $\mathrm{O}$ under 
solid-liquid phase transfer catalysis conditions. Thus, with 4-hydroxy-2-mercaptopyrimidine, where possible $\mathrm{S}, \mathrm{N}$, or $\mathrm{O}$ sites are available, the reaction afforded the S-substituted product. With 2-aminobenzothiazole or piperazine, the reaction yielded the corresponding $\mathrm{N}$-substituted 331a, b or the disubstituted products 332 [104] (Figure 118).

In a plan to study $\mathrm{C}$ - versus $\mathrm{O}$-alkylation of the pyrazoline derivatives, 3-methyl-1-phenyl-2-pyrazolin-5-one 333 was treated under PTC condition with different bromoorganic compounds such as benzyl bromide, 1,3-dibromopropane, methyl bromoacetate, bromoacetaldehyde, or diethylacetal as alkylating agents either in the absence or the presence of carbon disulphide and afforded numerous of C- or Oalkylated derivatives 334a-e, 335a-e [107] (Figure 119).

Reaction of 6-hydroxy-3,4-dihydroquinoline 337 and 1cyclohexyl-5-(4-bromo-butyl)-1,2,3,4-tetrazole 336 in a heterogeneous mixture of toluene $/ \mathrm{H}_{2} \mathrm{O}, \mathrm{K}_{2} \mathrm{CO}_{3}$ and TBACl as a catalyst gave 6-[4-(1-cyclohexyl-1,2,3,4-tetrazol-5-yl) butoxyl]-3,4-dihydrocarbostyril 338 in 99\% purity [108] (Figure 120).

5.2. Sulphonylation. 4,5-Dihydro-3,5-diaryl-1-(4-fluorophenylsulphonyl)pyrazoles 341 and 2-aryl-1-(4-fluorphenylsulphonyl)indoles 343 were prepared from reaction of 4-fluorophenylsulphonyl chloride 339 with the appropriate 4,5-dihydro-3,5-diarylpyrazoles 340 or 2-arylindoles 342 , via solid-liquid phase (THF/KOH/TBAB) [107] (Figure 121).

$\mathrm{N}$-Sulphonylation product 346 was prepared through treating 2-chloro-6,6-difluro-1,3-dioxolo[4,5-f]benzimidazole 344 with 3,5-dimethylisoxazole-4-sulphonyl chloride 345 via PTC protocol (toluene/aq. $\mathrm{K}_{2} \mathrm{CO}_{3} / \mathrm{TBAB}$ ) [109] (Figure 122).

5.3. N-Carbonylation. Carbonyldiimidazole 349 was prepared in $87 \%$ yield via reaction of imidazole 347 with phosgene 348 under PTC condition (chlorobenzene/NaOH/ tributylhexadecylphosphonium bromide (TBHDPB)) [110] (Figure 123).

5.4. N-Phosphorylation. Reaction of 1,3-thiazolidin-2-one 350 with S-sec-Bu-O-Et chlorophosphorothiolate 351 in the presence of $\mathrm{NaOH}$ and $\mathrm{N}$-dodecyl-N-methylephedrinium bromide gave s-sec-Bu O-Et(2-oxo-3-thiazolidinyl) phosphothiolate 352 in $84 \%$ yield [111] (Figure 124).

5.5. Elimination Reactions. $\beta$-Elimination of bromomethyl cyclic ketyl acetals 353 was carried out under solid-liquid phase ( $\mathrm{THF} / \mathrm{Bu}^{\mathrm{t}} \mathrm{OK} / \mathrm{Aliquat} 336$ ) to prepare the corresponding cyclic ketene acetals 354 [112] (Figure 125).

Reaction of 6-chloro-2-chloromethyl-3-nitroimidazo [1,2-b]pyridazine 355 with 3 equivalents of nitroalkane anion derivative 356 under liquid-liquid PTC protocol $\left(\mathrm{CH}_{2} \mathrm{Cl}_{2} / \mathrm{H}_{2} \mathrm{O} / \mathrm{TBA}(\mathrm{OH})\right)$ gave 3-nitroimidazo[1,2-b]pyridazines 357 bearing trisubstituted ethylenic double bond derivatives in a good yield [113] (Figure 126).

Vinylation of indole 358 with $\mathrm{ClCH}_{2} \mathrm{CH}_{2} \mathrm{Br}$ was achieved under PTC condition (toluene/KOH/18-crown-6) and afforded the corresponding $\mathrm{N}$-vinyl derivative 359 . Similarly, under the same reaction conditions N,S-divinyl derivatives 362 and 363 were prepared from 3-mercaptoindole 360 and 2-mercaptobenzimidazole 361, respectively [109] (Figure 127).

5.6. Condensation Reactions. Arylidenethiazolidin-4-ones 385 were synthesized from condensation reaction of 2thiono-N-( $m$-tolyl)thiazolidin-4-one $\mathbf{3 8 4}$ with aromatic or heteroaldehydes under solid-liquid PTC technique [104] (Figure 128).

5.7. Addition Reactions. Reaction of 2-thioxo-N-( $m$-tolyl) thiazolidin-4-one 386 with formaldehyde or some arylidenemalononitriles via PTC technique (dioxane/ $\mathrm{K}_{2} \mathrm{CO}_{3} /$ TBAB) afforded the corresponding 5-hydroxymethyl derivative $\mathbf{3 8 7}$ or Michael type adduct $\mathbf{3 8 8}$, respectively. While the addition reaction of cyanoacetamide to 5 -(p-methoxybenzylidene)-2-thioxo-N-(m-tolyl)thiazolidin-4-one $\mathbf{3 8 9}$ followed by condensation reaction under the same technique gave 7-anisyl-6-cyano-2-thioxo-3-( $m$-tolyl)thiazolo [4,5-b]pyridin-5-one 390 [104] (Figure 129).

5.8. Ring Expansion. Treatment of 5-alkyl(aryl)-3H-pyrrolin-2-ones 391 with dichlorocarbene using $\left(\mathrm{CHCl}_{3} / \mathrm{NaOH} /\right.$ $\mathrm{BTEAB})$ at $20-30^{\circ} \mathrm{C}$ afforded the intermediates 1-alkyl6,6-dichloro-2-azabicyclo[3.1.0]hexan-3-ones which directly rearranged and dehydrochlorinated to give 6-alkyl(aryl)-1phenyl-5-oxohydropyridin-2-ones 392 [110] (Figure 130).

Also, ring transformation of indole derivatives 393 has been induced by dichlorocarbene (aq. $\mathrm{KOH} / \mathrm{TBABSO}_{4} \mathrm{H}$ ) to produce 3-haloquinoline 394 in a good yield. Also, under similar conditions, reaction of 3-formyl-2-(3-chloro4-fluorophenyl)indole 395 with dichlorocarbene gave 3chloro-4-[2,2-dichloro-3-oxiranyl]-2-( $3^{\prime}$-chloro- $4^{\prime}$-fluorophenyl)quinoline 396. The evolved $\mathrm{HCl}$ gas has been eliminated during the reaction [111] (Figure 131).

5.9. Ring Opening. Treatment of benzosultams 400 with different disulphides under PTC technique $\left(\mathrm{CH}_{3} \mathrm{CN} / \mathrm{K}_{2} \mathrm{CO}_{3} /\right.$ $\mathrm{TBAHSO}_{4}$ ) gave the corresponding 2-methylamino-benzaldehyde dithioacetals 401 [24] (Figure 132).

\section{Abbreviations}

PTC: $\quad$ Phase transfer catalysis

TEBACl: Triethylbenzylammonium chloride

THF: Tetrahydrofuran

TBAB: Tetrabutylammonium bromide

DMF: Dimethylformamide

RT: $\quad$ Room temperature

DMSO: Dimethyl sulfoxide

BTEACl: Benzyltriethylammonium chloride

TBACl: Tetrabenzylammonium chloride

$\mathrm{TBAHSO}_{4}$ : Tetrabutylammonium hydrogen sulphate

CTPPCl: Crotyltriphenylphosphonium chloride

TBAHS: Tetrabutylammonium hydrogen sulphate

t-BuPz: Tert-butyl pyrazole

IPrPz: Isopropylpyrazole 
TEAI: Tetraethylammonium iodide

PEG: $\quad$ Polyethylene glycol

DCE: Dichloroethane

TBHDPB: Tributylhexadecylphosphonium bromide

TBA $(\mathrm{OH})$ : Tetrabutylammonium hydroxide

BTEAB: Benzyltriethylammonium bromide.

\section{Conflict of Interests}

The authors declare that they have no conflict of interests.

\section{References}

[1] M. Makosza and M. Fedoryński, "Catalysis in two-phase systems: phase transfer and related phenomena," Advances in Catalysis, vol. 35, pp. 375-422, 1987.

[2] E. V. Dehmlow and S. S. Dehmlow, Phase-Transfer Catalysis, Chemie, Weinheim, Germany, 3rd edition, 1993.

[3] C. M. Starks, C. L. Liotta, and M. Halpern, Phase Transfer Catalysis: Fundamentals, Applications and Industrial Perspectives, Chapman and Hall, New York, NY, USA, 1994.

[4] R. R. Nadendla, Indian Pharmacist, vol. 2, p. 13, 2003.

[5] M. Makosza, "Phase-transfer catalysis. A general green methodology in organic synthesis," Pure and Applied Chemistry, vol. 72, no. 7, pp. 1399-1403, 2000.

[6] J. Jarrouse and C. R. Hebd, Seances Acad Sci Ser C, vol. 232, p. 1424, 1951.

[7] H. Rath and U. Einsele, "Über die chemische Modifizierung der Zellulose durch Alkylierung," Melliand Textilber, vol. 40, p. 526, 1959.

[8] F. Bayer, German Patent, 959, 497, 1957.

[9] N. V. Gavaert, "Photo produce," Belgiam Patent, 602, 793, 1961.

[10] M. A. Iskenderov, V. V. Korshak, and S. V. Vinogradova, Vysokomol Soedin, vol. 4, p. 637, 1962.

[11] W. S. Port, British Patent, 912, 104, 1962.

[12] R. W. Kay, British Patent, 916, 772, 1963.

[13] S. Desikan and L. K. Doraiswamy, "Enhanced activity of polymer-supported phase transfer catalysts," Chemical Engineering Science, vol. 55, no. 24, pp. 6119-6127, 2000.

[14] T. Shioiri, "Chiral phase transfer catalysis," in Handbook of Phase-Transfer Catalysis, Y. Sasson and R. Neumann, Eds., chapter 14, Blackie Academic \& Professional, London, UK, 1997.

[15] M. Makosza, "Two-phase reactions in the chemistry of carbanions and halocarbenes. A useful tool in organic synthesis," Pure and Applied Chemistry, vol. 43, p. 439, 1975.

[16] M. Makosza and I. Krylowa, "Remarks on the mechanism of phase-transfer catalyzed carbanion generation in two-phase systems," Tetrahedron, vol. 55, pp. 6395-6402, 1999.

[17] H. Abdel-Ghany, A. M. El-Sayed, and A. K. El-Shafei, "Synthesis of pyrrole, pyridinone and pyrimidinone derivatives using PTC conditions," Synthetic Communications, vol. 25, no. 8, pp. 11191131, 1995.

[18] C. G. Dave and V. A. Parikh, "Heterocyclization using phase transfer catalysis: a simple and convenient synthesis of 2amino-1-aryl-5-oxo-4,5-dihydro-1H-pyrrole-3-carbonitriles," Synthetic Communications, vol. 31, no. 9, pp. 1301-1306, 2001.

[19] A. M. El-Sayed, Trends in Heterocycles, vol. 9, p. 33, 2003.

[20] D. Albanese, D. Landini, and M. Penso, "Synthesis of 2-amino acids via selective mono- $\mathrm{N}$-alkylation of trichloroacetamide by 2-bromo carboxylic esters under solid-liquid phase-transfer catalysis conditions," Journal of Organic Chemistry, vol. 57, no. 5, pp. 1603-1605, 1992.

[21] J. Casas, R. Grigg, C. Nájera, and J. M. Sansano, “The effect of phase-transfer catalysis in the 1,3-dipolar cycloaddition reactions of azomethine ylides - Synthesis of substituted prolines using AgOAc and inorganic base in substoichiometric amounts," European Journal of Organic Chemistry, no. 10, pp. 1971-1982, 2001.

[22] O. A. Abd Allah and A. M. El-Sayed, "Application of phase transfer catalysis in heterocyclic synthesis: synthesis of some new polyfunctional thiophenes, thiopyrans, pyridines and oxazines," Phosphorus, Sulfur and Silicon and Related Elements, vol. 177, no. 5, pp. 1291-1301, 2002.

[23] A. K. El-Shafei, H. Abdel-Ghany, A. A. Sultan, and M. M. ElSaghier, "Synthesis of thieno (2,3-b) thiophenes and related structures," Phosphorus, Sulfur, and Silicon and the Related Elements, vol. 73, pp. 15-25, 1992.

[24] H. Modrzejewska and K. Wojciechowski, "Synthesis and reactions of 3-alkylsulfanyl-1,3-dihydro-2,1-benzisothiazole 2,2dioxides," Tetrahedron, vol. 61, no. 37, pp. 8848-8854, 2005.

[25] A. Khodairy, "Synthesis of fused and spiro heterocyclic compounds derived from 3,5-pyrazolidinedione derivatives," Phosphorus, Sulfur and Silicon and Related Elements, vol. 160, pp. 159-180, 2000.

[26] D. Bogdal and M. Warzala, "Microwave-assisted preparation of benzo[b]furans under solventless phase-transfer catalytic conditions," Tetrahedron, vol. 56, no. 44, pp. 8769-8773, 2000.

[27] M. Makosza, J. Przyborowski, R. Klajn, and A. Kwast, "Simple synthesis of 2-substituted tetrahydrofuran-3-carbonitriles," Synlett, no. 12, pp. 1773-1774, 2000.

[28] K. S. Krishna Murthy, B. Rajitha, and M. Kanakalingeswara Rao, "Synthesis of biologically active angularly fused bisaroylbenzodifurans by PTC and solvent free microwave irradiation," Indian Journal of Chemistry B, vol. 42, no. 2, pp. 425-428, 2003.

[29] R. D. Shah, "Phase transfer catalysis assisted thorpe reaction for the synthesis of 3-aminothiophene-2-carboxylates," E-Journal of Chemistry, vol. 8, no. 1, pp. 368-372, 2011.

[30] W. He, B.-L. Zhang, Z.-J. Li, and S.-Y. Zhang, "PTC-promoted Japp-Klingmann reaction for the synthesis of indole derivatives," Synthetic Communications, vol. 35, no. 10, pp. 1359-1368, 2005.

[31] T. Cholakova, Y. Zagraniarsky, S. Simova, S. Varbanov, and A. Dobrev, "A simple synthesis of dimethylphosphinyl-substituted tetrahydropyrroles," Phosphorus, Sulfur and Silicon and the Related Elements, vol. 180, no. 7, pp. 1721-1728, 2005.

[32] A. Khodairy and A. M. El-Saghier, "Thieno[2,3-b]thiophenes: part 7. Some heterocyclization reactions with ethyl 3,4-diamino5-cyanothieno[2,3-b]thiophene-2-carboxylate," Acta Chimica Slovenica, vol. 58, no. 2, pp. 360-366, 2011.

[33] M. Y. Ait Itto, A. Hasnaoui, A. Riahi, and J.-P. Lavergne, "Regioselective synthesis of new biheterocyclic triazepines," Tetrahedron Letters, vol. 38, no. 12, pp. 2087-2090, 1997.

[34] G. Molteni, A. Ponti, and M. Orlandi, "Uncommon aqueous media for nitrilimine cycloadditions. I. Synthetic and mechanistic aspects in the formation of 1-aryl-5-substituted-4,5dihydropyrazoles," New Journal of Chemistry, vol. 26, no. 10, pp. 1340-1345, 2002.

[35] B. W. LeBlanc and B. S. Jursic, "Preparation of 5-alkylthio and 5-arylthiotetrazoles from thiocyanates using phase transfer catalysis," Synthetic Communications, vol. 28, no. 19, pp. 3591$3599,1998$. 
[36] A. Ogihara, H. Sakai, N. Matsui, and H. Miyazaki, PCT International Applications WO0276,958 (Cl.C/32 ), 2002.

[37] P. Sharma, A. Kumar, and M. Sharma, "Generation of 4,6-dimethyl-5-[2-(2-methylprop-1-enyl)-1H-benzimidazol1-yl]pyrimidine-2(5H) -thiones under kinetically controlled phase transfer catalysis conditions," Journal of Molecular Catalysis A, vol. 237, no. 1-2, pp. 191-198, 2005.

[38] A. M. Youssef, Egyptian Journal of Chemistry, vol. 45, p. 777, 2002.

[39] M. F. Farhat, A. M. M. El-Saghier, M. A. Makhlouf, K. M. Kreddan, and A. B. Elmezoughi, "Ketene N,S-acetals in heterocyclic synthesis: part 1: synthesis of $\mathrm{N}$-phenyl-2-ylidene and 2,5-diylidene-4-thiazolidinone derivatives," Journal of Sulfur Chemistry, vol. 28, no. 6, pp. 563-572, 2007.

[40] M. Fedoryński, M. Jezierska-Zięba, and B. Kąkol, "Phase transfer catalysis in pharmaceutical industry-where are we?" Acta Poloniae Pharmaceutica, vol. 65, pp. 647-654, 2008.

[41] A. K. Khalil, "Phase-transfer catalyzed alkylation and cycloalkylation of 2-mercaptoquinazolin-4(3H)-one," Phosphorus, Sulfur and Silicon and the Related Elements, vol. 180, no. 11, pp. 25332541, 2005.

[42] M. K. Abou El-Regal, A. A. Abdalha, M. A. El-Kassaby, and A. T. Ali, "Synthesis of new thiohydantoin derivatives under phase transfer catalysis," Phosphorus, Sulfur and Silicon and the Related Elements, vol. 182, no. 4, pp. 845-851, 2007.

[43] S. El-Metwally and A. K. Khalil, "Reactions of 1,3-diphenyl2-pyrazolin-5-one and 4-amino-1,5-dimethyl-2- phenyl- $1 \mathrm{H}$ pyrazol-3(2H)-one. Synthesis of some new pyrazoles and pyrazolones," Acta Chimica Slovenica, vol. 57, no. 4, pp. 941-947, 2010.

[44] O. Atsushi, S. Hiroshi, M. Nobuo, and M. Hidekazu, PCT International Applications, WO 0276, 958, 2002.

[45] M. V. Zatsepina, T. V. Artamonova, and G. I. Koldobskii, “Tetrazoles: LII. Synthesis of functionally substituted tetrazoles from benzene-1,3,5-tricarboxylic acid derivatives," Russian Journal of Organic Chemistry, vol. 44, no. 4, pp. 577-581, 2008.

[46] A. M. M. El-Saghier, "Simple one-pot synthesis of thieno[2,3b]thiophene derivatives under solid-liquid PTC conditions. Useful starting material for the synthesis of biological active compounds," Bulletin of the Chemical Society of Japan, vol. 66, no. 7, pp. 2011-2015, 1993.

[47] K. C. Majumdar, S. Saha, and A. T. Khan, "Indian Journal of Chemistry B," vol. 33, p. 216, 1994.

[48] R. M. Butnariu and I. I. Mangalagiu, "New pyridazine derivatives: Synthesis, chemistry and biological activity," Bioorganic and Medicinal Chemistry, vol. 17, no. 7, pp. 2823-2829, 2009.

[49] A. M. El-Sayed, "One-pot synthesis of pyridines, thienopyridines, pyrrolothienopyridines and $(1,8)$ naphthyridines under phase-transfer catalysis conditions," Phosphorus, Sulfur and Silicon and Related Elements, vol. 163, pp. 29-40, 2000.

[50] A. K. El-Shafei, A. M. Soliman, A. A. Sultan, and A. M. M. El-Saghier, "Synthesis of some new fused heterocycles containing a bridgehead nitrogen atom under ptc conditionsa new application of cyanoketene s,s-acetals," Gazzetta Chimica Italiana, vol. 125, p. 115, 1995.

[51] A. Khodairy and A. M. El-Sayed, "Synthetic studies on the synthesis of pyridine, $\alpha$-pyran, $\alpha$-thiopyran, and Thienothiopyranopyrrol derivatives using PTC technique," Synthetic Communications, vol. 31, no. 4, pp. 475-486, 2001.

[52] G. A. El-Saraf, A. M. El-Sayed, and A. M. M. El-Saghier, "One-pot PTC synthesis of polyfused pyrazoles," Heteroatom Chemistry, vol. 14, no. 3, pp. 211-217, 2003.
[53] P. Sampath Rao, K. Vishnu Vardhan Reddy, and D. Ashok, "An efficient synthesis of dibenzoyl dialkyl benzodifurans under phase transfer catalysis conditions and their antifeedant activity," Indian Journal of Heterocyclic Chemistry, vol. 8, no. 1, pp. 51-54, 1998.

[54] P. Sampath Rao, K. Vishnu Vardhan Reddy, and D. Ashok, "Synthesis of 2,6-dibenzoyl-3,5-distyryl benzo (1,2-b: 5,4-b/)difurans as potential antifeedants," Indian Journal of Heterocyclic Chemistry, vol. 7, no. 4, pp. 293-294, 1998.

[55] P. Sampath Rao, K. Vishnu Vardhan Reddy, and D. Ashok, "An efficient synthesis of dibenzoyl dialkyl benzodifurans under phase transfer catalysis conditions and their antifeedant activity," Indian Journal of Heterocyclic Chemistry, vol. 8, no. 1, pp. 51-54, 1998.

[56] K. S. Krishna Murthy, B. Rajitha, M. Kanakalingeswara Rao, T. Raja Komuraiah, and S. M. Reddy, "Facile synthesis of biologically active linear bisaroyl benzodifurans by ptc and solvent free microwave irradiation," Heterocyclic Communications, vol. 8, no. 2, pp. 179-186, 2002.

[57] A. M. El-Sayed, H. Abdel-Ghany, and A. M. M. El-Saghier, "A novel synthesis of pyrano(2,3-c)-, 1,3-oxazino(2,3 b),1,2,4- triazolo(3,4-b)-, oxazolo(2,3-b)-, furano(3,2-c)-, and 3substituted- (1,5)benzodiazepin-2-ones," Synthetic Communications, vol. 29, no. 20, pp. 3561-3572, 1999.

[58] A. Khodairy, H. Abdel-Ghany, A. M. El-Sayed, and H. Salah, "Synthesis of new fused and spiro 1,5-benzodiazepines," Journal of the Chinese Chemical Society, vol. 50, no. 6, pp. 1195-1198, 2003.

[59] A. M. Soliman, A. A. Sultan, and A. K. El-Shafei, "Synthesis of some new dispiro[dipyrano- $\left(2,41: 6,4^{\prime \prime}\right)$ bidithiolo( 4,5 b:4I,5I-e)-4,8-benzoquinones]," Monatshefte für Chemie Chemical Monthly, vol. 126, no. 5, pp. 615-619, 1995.

[60] A. A. Sultan, "Synthesis of some condensed s-triazole heterocycles using phase-transfer catalysis technique," Phosphorus, Sulfur, and Silicon and the Related Elements, vol. 105, pp. 123-127, 1995.

[61] A. M. M. El-Saghier, "A novel one-pot synthesis of polyfunctionally substituted thiopyrano(2,3-b)pyridine and pyrido(2,3b)pyridine derivatives under solid-liquid phase-transfer catalysis," Phosphorus, Sulfur and Silicon and Related Elements, vol. 177, no. 5, pp. 1213-1221, 2002.

[62] C. Bezergiannidou-Baloucts, K. E. Litinas, E. M. Xenikaki, and D. N. Nicolaides, "Reactions of 7-(Methoxyimino)-4-methyl$2 H$-chromene-2,8(7H)-dione with Phosphorus Ylides. Synthesis of 2-Substituted 6-Methyl-8H-benzopyrano[7,8-d] oxazol-8ones," Liebigs Annalen Der Chemie, vol. 1993, pp. 1175-1177, 1993.

[63] H. A. Ghany, "Synthesis of new spiro heterocyclic compounds derived from rhodanine derivatives," Phosphorus, Sulfur and Silicon and Related Elements, vol. 122, pp. 173-180, 1997.

[64] H. Abdel-Ghany, A. M. El-Sayed, A. Khodairy, and H. Salah, "Synthesis of new 3-substituted and spiro 1,5-benzodiazepin2-ones under phase-transfer catalysis conditions," Synthetic Communications, vol. 31, no. 16, pp. 2523-2535, 2001.

[65] E. Diez-Barra, A. de la Hoz, A. Loupy, and A. Sanchez-Migallon, "Selective alkylation of pyrrole by phase transfer catalysis in the absence of solvent," Journal of Heterocyclic Chemistry, vol. 31, pp. 1715-1717, 1994.

[66] A. Nakmura and K. Takamoto, Japanese Kokai Tokkyo Koho JP, 171,379, (Cl.C07D417/12), 2003.

[67] A. Nakamura and K. Takamoto, Japanese Kokai Tokkyo Koho JP, 138, 079, (Cl.C07D209/48), 2002. 
[68] G. Guazzelli and R. Settambolo, "4-Indolylbutanals from rhodium-catalyzed hydroformylation of allylindoles as precursors of benzofused indolizines," Tetrahedron Letters, vol. 48, no. 34, pp. 6034-6038, 2007.

[69] R. Settambolo, S. Miniati, and R. Lazzaroni, "One pot hydroformylation/intramolecular aldol condensation reactions of 1allyl-2-carbonylpyrroles: a new entry into hydroindolizines synthesis," Synthetic Communications, vol. 33, no. 17, pp. 29532961, 2003.

[70] R. Settambolo, M. Mariani, and A. Caiazzo, "Synthesis of 1,2and 1,3-divinylpyrrole," Journal of Organic Chemistry, vol. 63, no. 26, pp. 10022-10026, 1998.

[71] P. De La Cruz, A. De La Hoz, L. M. Font, F. Langa, and M. C. Pérez-Rodríguez, "Solvent-free phase transfer catalysis under microwaves in fullerene chemistry. A convenient preparation of N-alkylpyrrolidino[60]fullerenes," Tetrahedron Letters, vol. 39, no. 33, pp. 6053-6056, 1998.

[72] I. Almena, E. Díez-Barra, A. De La Hoz, J. Ruiz, A. SánchezMigallón, and J. Elguero, "Alkylation and arylation of pyrazoles under solvent-free conditions: conventional heating versus microwave irradiation," Journal of Heterocyclic Chemistry, vol. 35, no. 6, pp. 1263-1268, 1998.

[73] Inc. Hoffman La-Roch, NJ. Nutely,United States Patent, Patent No. US 6,326,501 B1, 2001.

[74] A. Jończyk, E. Nawrot, and M. Kisielewski, "Reactions of some nitrogen heterocycles with chlorodifluoromethane under conditions of phase-transfer catalysis," Journal of Fluorine Chemistry, vol. 126, no. 11-12, pp. 1587-1591, 2005.

[75] P. Barraja, P. Diana, A. Carbone, and G. Cirrincione, "Nucleophilic reactions in the indole series: displacement of bromine under phase transfer catalysis," Tetrahedron, vol. 64, no. 51, pp. 11625-11631, 2008.

[76] C. Barrett, B. Choudhury, A. Natansohn, and P. Rochon, "Azocarbazole polymethacrylates as single-component electrooptic materials," Macromolecules, vol. 31, no. 15, pp. 4845-4851, 1998.

[77] I. Almena, E. Díez-Barra, A. De La Hoz, J. Ruiz, A. SánchezMigallón, and J. Elguero, "Alkylation and arylation of pyrazoles under solvent-free conditions: conventional heating versus microwave irradiation," Journal of Heterocyclic Chemistry, vol. 35, no. 6, pp. 1263-1268, 1998.

[78] F. L. Tang, Z. H. Wang, W. L. Jia, Y. M. Xu, and J. T. Wang, "Synthesis of diorganotin derivatives containing asymmetric disubstituted bis(pyrazol-1-yl)methanes: X-ray crystal structures of (3-isopropylpyrazol-1-yl-5' -isopropylpyrazol-1yl)methane diphenyltin(IV) dibromide and bis(5-isopropylpyrazol-1-yl)methane diphenyltin(IV) dibromide," Polyhedron, vol. 19, pp. 381-387, 2000.

[79] V. N. Pathak, C. K. Oza, R. Pathak et al., "Fluorophenylsulphonylation of 4,5-dihydro-3,5-diarylpyrazoles and 2-arylindoles via solid-liquid phase transfer catalysis," Indian Journal of Heterocyclic Chemistry, vol. 7, no. 3, pp. 241-242, 1998.

[80] E. Arranz, J. A. Díaz, E. Morante, C. Pérez, and S. Vega, "Preparation and regiochemical assignments of new pyrazolo[3,4-c] [2,1] benzothiazepines," Journal of Heterocyclic Chemistry, vol. 33, no. 1, pp. 151-156, 1996.

[81] M. L. Taha, H. B. Lazrek, J. L. Barascut, and J. L. Imbach, "Synthesis of some 4-substituted 1-[(2-hydroxyethoxy)methyl]pyrazolo[3,4-d]pyrimidines," Bulletin des Sociétés Chimiques Belges, vol. 105, pp. 279-285, 1996.

[82] H. B. Lazrek, M. Taourirte, J. L. Barascut, and J. L. Imbach, Bulletin Des Sociétés Chimiques Belges, vol. 105, p. 391, 1996.
[83] E. Diez-Barra, A. De La Hoz, A. Sanchez-Migallon, and J. Tejeda, "Alkylation of imidazole by solid-liquid phase transfer catalysis in the absence of solvent," Synthetic Communications, vol. 23, no. 13, pp. 1783-1786, 1993.

[84] X. Chen, X. Wang, H. Wang, H. Lian, Y. Pan, and Y. Shi, "N-alkylation of 2-methyl-2-imidazolines by phase transfer catalysis without solvent," Synthetic Communications, vol. 29, no. 17, pp. 3025-3030, 1999.

[85] X. Chen, X. Wang, H. Wang, H. Lian, Y. Pan, and Y. Shi, "ChemInform abstract: N-Alkylation of 2-Methyl-2-imidazolines by phase transfer catalysis without solvent," Cheminform, vol. 30, no. $43,1999$.

[86] E. V. Dehmlow, R. Richter, and A. B. Zhivich, Journal of Chemical Research S, vol. 504, 1993.

[87] S. Khabnadideh, Z. Rezaei, A. Khalafi-Nezhad, R. Bahrinajafi, R. Mohamadi, and A. A. Farrokhroz, "Synthesis of N-alkylated derivatives of imidazole as antibacterial agents," Bioorganic and Medicinal Chemistry Letters, vol. 13, no. 17, pp. 2863-2865, 2003.

[88] K. Ramaiah, P. K. Dubey, D. Eswara Rao et al., "Synthesis and spectral properties of a variety of 2-styrylbenzimidazoles," Indian Journal of Chemistry B, vol. 39, no. 12, pp. 904-914, 2000.

[89] S. Khabnadideh, Z. Rezaei, A. Khalafi-Nezhad, R. Bahrinajafi, R. Mohamadi, and A. A. Farrokhroz, "Synthesis of N-alkylated derivatives of imidazole as antibacterial agents," Bioorganic and Medicinal Chemistry Letters, vol. 13, no. 17, pp. 2863-2865, 2003.

[90] E. V. Dehmlow, J. Bollhofer, and G. Thye, "Journal of Chemical Research S," vol. 113, 2001.

[91] A. Abenhaim, "Selective alkylations of 1,2,4-triazole and benzotriazole in the absence of solvent," Heterocycles, vol. 38, no. 4, p. 793, 1994.

[92] I. P. Beletskaya, D. V. Davydov, M. S. Gorovoi, and S. V. Kardashov, "Selective N(1)-arylation of benzotriazole with activated aryl halides under conditions of phase transfer catalysis," Russian Chemical Bulletin, vol. 48, no. 8, pp. 1533-1536, 1999.

[93] C. G. Oliva, N. Jagerovic, P. Goya et al., "N-substituted1,2,3-triazoles: Synthesis, characterization and evaluation as cannabinoid ligands," Arkivoc, vol. 2010, no. 2, pp. 127-147, 2010.

[94] K. Waisser, J. Kunes, A. Hrabalek, M. Machacek, and Z. Odlerova, "New groups of potential antituberculotics: 5Alkylthio-1-aryltetrazoles," Collection of Czechoslovak Chemical Communications, vol. 61, p. 791, 1996.

[95] M. L. Cerrada, J. Elguero, J. De La Fuente, C. Pardo, and M. Ramos, "Synthesis of p-nitrophenylazoles by phase transfer catalysis without solvent," Synthetic Communications, vol. 23, no. 14, pp. 1947-1952, 1993.

[96] Y. V. Poplavskaya, L. V. Alam, and G. I. Koldobskii, “Tetrazoles: XLI. Alkylation of 1-aryltetrazol-5-ones," Russian Journal of Organic Chemistry, vol. 36, no. 12, pp. 1793-1799, 2000.

[97] K. Aritomo, T. Wada, and M. Sekine, "Alkylation of 6-Nacylated adenosine derivatives by the use of phase transfer catalysis," Journal of the Chemical Society, no. 14, pp. 1837-1844, 1995.

[98] H. Rodríguez, R. Pérez, M. Suárez, A. Lam, N. Cabrales, and A. Loupy, "Alkylation of some pyrimidine and purine derivatives using microwave-assisted methods," Heterocycles, vol. 55, no. 2, pp. 291-302, 2001.

[99] L. Zheng, W. Xi-cun, Y. Jing-ya, and Z. Kexueban, Journal of Northwest Normal University, vol. 37, p. 54, 2001.

[100] O. S. Attaryan, A. O. Baltayan, R. E. Sagatelyan, and K. T. Takmazyan, "Synthesis of 1-(2-aminoethyl)pyrazoles under phase-transfer catalysis," Russian Journal of General Chemistry, vol. 78, no. 1, pp. 136-138, 2008. 
[101] O. S. Attarian, S. G. Matsoyan, and S. S. Martirosyan, "Synthesis of N-vinylpyrazoles," Chemistry of Heterocyclic Compounds, vol. 41, no. 4, pp. 452-455, 2005.

[102] M. Majdoub, A. Loupy, A. Petit, and S. Roudesli, "Coupling focused microwaves and solvent-free phase transfer catalysis: application to the synthesis of new furanic diethers," Tetrahedron, vol. 52, no. 2, pp. 617-628, 1996.

[103] T. M. Slaghek, A. H. van Oijen, A. A. M. Maas, J. P. Kamerling, and J. F. G. Vliegenthart, "Synthesis of structural elements of the capsular polysaccharides of Streptococcus pneumoniae types 6A and 6B," Carbohydrate Research, vol. 207, no. 2, pp. 237-248, 1990.

[104] A. K. El-Shafei, A. M. El-Sayed, A. A. Sultan, and H. AbdelGhany, Gazzetta Chimica Italiana, vol. 120, p. 197, 1990.

[105] S.-S. Jew, Y.-J. Lee, J. Lee et al., "Highly enantioselectlve phase-transfer-catalytic alkylation of 2-phenyl-2-oxazoline-4carboxylic acid tert-butyl ester for the asymmetric synthesis of $\alpha$-alkyl serines," Angewandte Chemie - International Edition, vol. 43, no. 18, pp. 2382-2385, 2004.

[106] T. Tamura and E. Ryukoku, Japanese Kokai Tokkyo Koho JP, 128, 688 (Cl. CO7F 9/6539), 2003.

[107] S. A. Shiba, N. S. Harb, M. El-Kassaby, M. A. Hassan, and N. M. Abouelregal, "Facile synthesis of 4-substituted 2-pyrazolin5-ones under phase transfer catalysis," Phosphorus, Sulfur, and Silicon and the Related Elements, vol. 104, pp. 15-20, 1995.

[108] USPTO Applicaton \#: \#20090176826-Class: 514312 (USPTO).

[109] E. Abele, O. Dzenitis, K. Rubina, and E. Lukevics, "Synthesis of $\mathrm{N}$ - and S-vinyl derivatives of heteroaromatic compounds using phase-transfer catalysis," Chemistry of Heterocyclic Compounds, vol. 38, no. 6, pp. 682-685, 2002.

[110] A. Yu Egorova, V. A. Sedavkina, and Z. Y. Temofeyeva, "Interaction of 1,5-substituted pyrrolin-2-ones with dichlorocarbene under phase transfer catalysis conditions," Molecules, vol. 5, pp. 1082-1084, 2000.

[111] C. I. Krishna, J. Renuka, and S. Arora, Journal of the Indian Chemical Society, vol. 70, p. 567, 1993.

[112] W. J. Bailey and L.-L. Zhou, "A new elimination with phasetransfer catalysis for cyclic ketene acetals," Tetrahedron Letters, vol. 32, no. 12, pp. 1539-1540, 1991.

[113] T. Terme, C. Galtier, J. Maldonado, M. P. Crozet, A. Gueiffier, and P. Vanelle, "Synthesis of 2-substituted-3-nitroimidazo[1,2b]pyridazines as potential biologically active agents," Journal of Heterocyclic Chemistry, vol. 39, no. 1, pp. 173-177, 2002. 

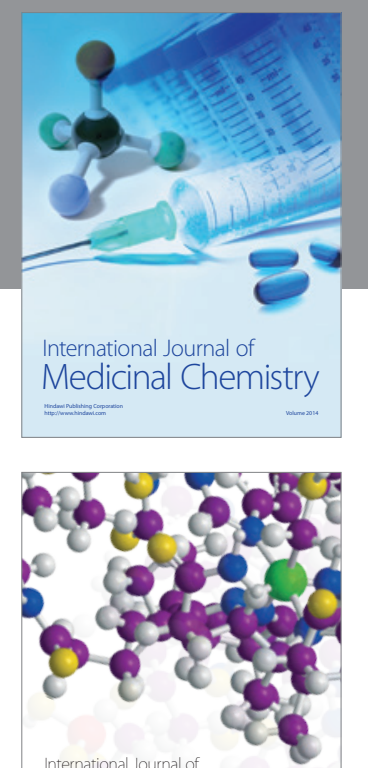

\section{Carbohydrate} Chemistry

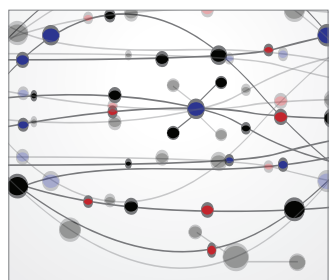

The Scientific World Journal
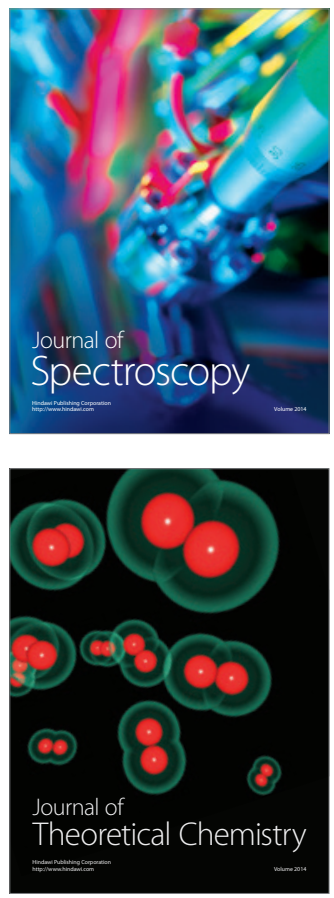
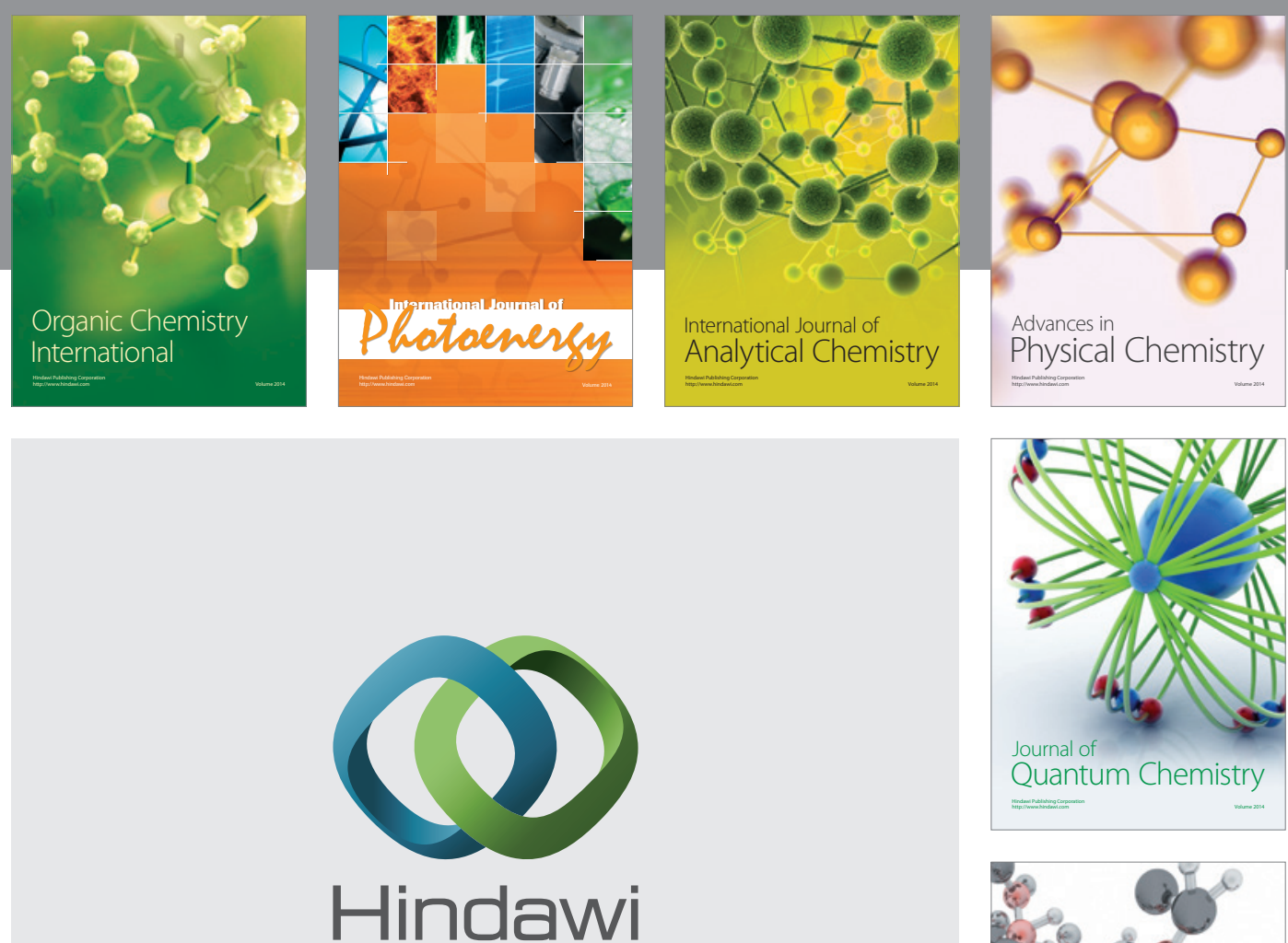

Submit your manuscripts at

http://www.hindawi.com

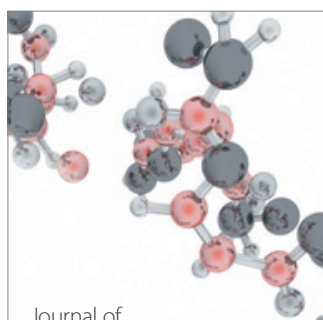

Analytical Methods

in Chemistry

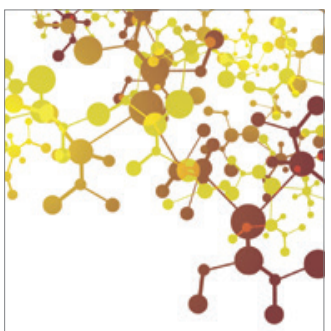

Journal of

Applied Chemistry

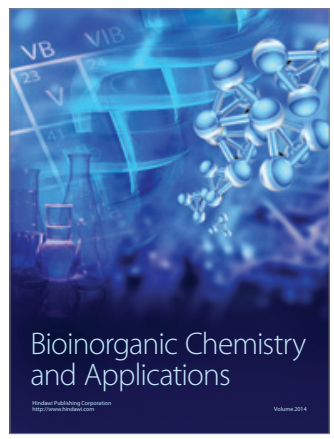

Inorganic Chemistry
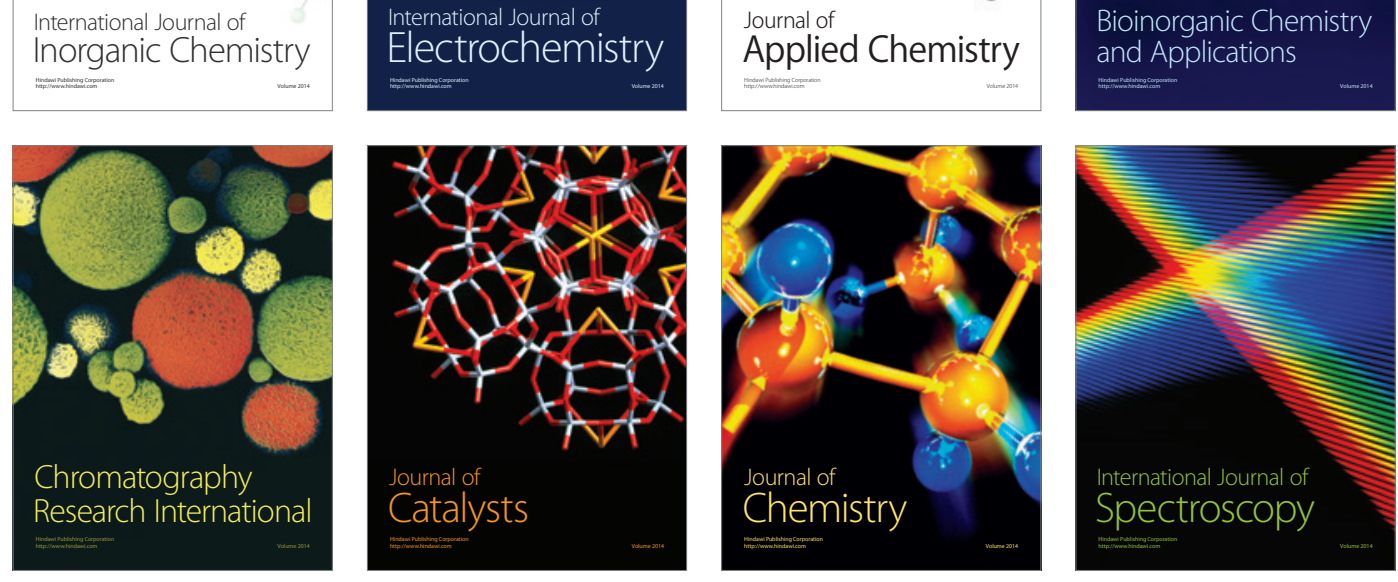